\title{
Biogenesis and structure of a Type VI secretion membrane core complex
}

\author{
Eric Durand ${ }^{1,2,3,4,5^{*}}$, Van Son Nguyen ${ }^{2,5^{*}}$, Abdelrahim Zoued ${ }^{1 *}$, Laureen Logger ${ }^{1}$, Gérard \\ Péhau-Arnaudet ${ }^{4}$, Marie-Stéphanie Aschtgen ${ }^{1}$, Silvia Spinelli ${ }^{2,5}$, Aline Desmyter ${ }^{2,5}$, \\ Benjamin Bardiaux $^{4,6}$, Annick Dujeancourt ${ }^{3,4}$, Alain Roussel ${ }^{2,5}$, Christian Cambillau ${ }^{2,5}$, \\ Eric Cascales ${ }^{1}$ and Rémi Fronzes ${ }^{3,4}$
}

1 Laboratoire d'Ingénierie des Systèmes Macromoléculaires, Aix-Marseille Université CNRS, UMR 7255, 31 Chemin Joseph Aiguier, 13402 Marseille Cedex20, France

2 Architecture et Fonction des Macromolécules Biologiques, CNRS, UMR 7257, Campus de Luminy, Case 932, 13288 Marseille Cedex 09, France

${ }^{3}$ G5 Biologie structurale de la sécrétion bactérienne, Institut Pasteur, 25-28 rue du Docteur Roux, 75015 Paris, France.

${ }^{4}$ UMR 3528, CNRS, Institut Pasteur, 25-28 rue du Docteur Roux, 75015 Paris, France

5 AFMB, Aix-Marseille Université, IHU Méditerranée Infection, Campus de Luminy, Case 932, 13288 Marseille Cedex 09, France

${ }^{6}$ Unité de Bioinformatique Structurale, Institut Pasteur, 25-28 rue du Docteur Roux, 75015 Paris, France

* These authors contributed equally to this work

Correspondence and requests for materials should be addressed to C.C. (cambillau@afmb.univ-mrs.fr), E.C. (cascales@imm.cnrs.fr) or R.F. (remi.fronzes@pasteur.fr)

Bacteria share their ecological niches with other microbes. The bacterial Type VI secretion system is one of the key players for microbial competition, as well as an important virulence determinant during bacterial infections. It assembles a nanocrossbow-like structure that propels an arrow made of Hcp tube and VgrG spike into the cytoplasm of the attacker cell and punctures the prey's cell wall. The nano-crossbow is stably anchored to the cell envelope of the attacker by a membrane core complex. Here, we show that this complex is assembled by the sequential addition of three proteins -TssJ, TssM and TssL- and present a $11.6 \AA$ resolution structure of the fully assembled complex, determined by negative stain electron microscopy. With overall $\mathrm{C5}$ symmetry, this 1.7-megadalton complex comprises a large base in the cytoplasm. It extends in the periplasm via 10 arches to form a double-ring structure containing the $\mathrm{C}$ terminal domain of TssM (TssM $\left.\mathrm{T}_{\mathrm{ct}}\right)$ and TssJ that is anchored in the outer membrane. The crystal structure of the $T_{s s} M_{c t}-T s s J$ complex coupled to whole-cell accessibility studies suggest that large conformational changes induce transient pore formation in the outer membrane allowing passage of the attacking Hcp tube/VgrG spike. 
In the environment, bacteria have evolved collaborative or aggressive mechanisms to communicate, exchange information and chemicals or compete for space and resources ${ }^{1-3}$. One of the main weapons of bacterial conflicts is a multiprotein device called the Type VI secretion system (T6SS) that is assembled in the attacker bacterium ${ }^{4}$. The T6SS is a versatile nanomachine than can deliver toxin proteins directly not only into prey prokaryotes, but also into eukaryotic cells during bacterial infections ${ }^{3,5-9}$. Anti-host activities have been shown to inhibit phagocytosis and therefore to disable macrophages, while the anti-bacterial activities allow the bacterium to destroy competitors and to have a privileged access to the niche, to nutrients or to new DNA ${ }^{3,9,10}$. The T6SS is constituted of 13 different proteins, encoded by genes that are usually clustered ${ }^{11}$. It assembles a tubular puncturing device that is evolutionarily, structurally and functionally similar to the tail of contractile bacteriophages. Its sheath is a hundreds-nanometer-long tubular structure that extends in the cytoplasm and is built by the polymerization of TssBC building blocks ${ }^{12-14}$. It is assembled on an assembly platform, the baseplate ${ }^{13,15-17}$ and maintained in an extended, metastable conformation ${ }^{16-18}$. The attacking arrow, wrapped by the sheath, comprises an inner tube that is built by stacked Hcp hexameric rings ${ }^{19}$ and tipped by a puncturing spike composed of $\mathrm{VgrG}^{20}$. Upon contact with the prey, structural rearrangements of the sheath subunits induce its contraction and propulsion of the Hcp tube/VgrG spike towards the target cell allowing toxin delivery ${ }^{16,17,21}$. The phage-like T6SS tail is anchored to the attacker cell membrane by a trans-envelope complex $^{22}$. This membrane complex does not only serve as a docking station but has been proposed to be used as a channel for the passage of the inner tube after sheath contraction, and therefore prevents membrane damage in the attacker ${ }^{16,17}$. The membrane core complex of the T6SS (i.e., the minimal module required to function and conserved in all T6SS) is composed of the TssL and TssM inner membrane proteins and the TssJ outer membrane lipoprotein ${ }^{15-17}$, ${ }^{22-26}$. These proteins are connected through a network of interactions between TssM and TssL, and TssM and TssJ $\mathrm{J}^{22,24,25}$. Although the localization and topology of these subunits, their interactions, and the crystal structures of the soluble domains of TssJ and TssL have been described $^{17,22-29}$, we still lack crucial information on the biogenesis and overall architecture of this complex and how it is used as a channel during T6SS action.

We first sought to determine the assembly pathway of the entero-aggregative Escherichia coli (EAEC) T6SS membrane core complex. Strains producing fluorescentlylabeled T6SS membrane subunits were engineered. The sequence encoding the super-folder Green Fluorescent Protein (sf-GFP) was inserted upstream the stop codon of the tss $J$ gene or downstream the start codon of the $t s s L$ and $t s s M$ genes. In these constructs, the fusion proteins were produced from their original chromosomal loci. Hcp release and anti-bacterial assays

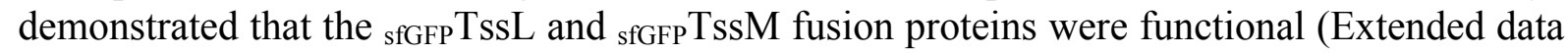
Fig. 1a). By contrast, strains producing TssJ $J_{\text {sfGFP }}$ or $\mathrm{Tss}_{\mathrm{mCherry}}$ had a non-functional T6SS (Extended data Fig. 1b). Fluorescence microscopy analyses showed that sfGFPTssL and sfGFPTssM cluster at discrete positions at the cell periphery, in agreement with their membrane localization (Fig. 1a and Extended data Fig. 1c). These foci are stable and static (Fig. 1a and Extended data Fig. 1d). Statistical analyses further showed that one or two foci are observable in cells expressing the T6SS (Fig. 1b) and that these clusters are randomly distributed around

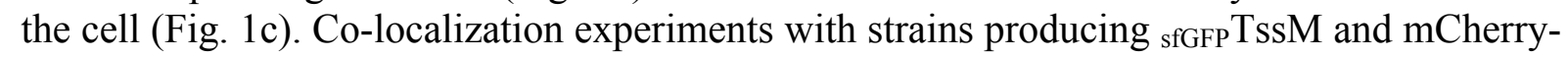
tagged TssL showed that the two subunits are present in the foci, demonstrating that each focus corresponds to the position of an assembled membrane complex (Fig. 1d). To test whether these foci are used to anchor the phage-like tail tubular structure, mCherry was fused to the $t s s B$ sheath gene, at its original chromosomal locus in the strain producing ${ }_{\text {sfGFP }}$ TssL. 
Time-lapse recordings showed that T6SS sheathes polymerize and extend from the membrane complex (Fig. 1e). Based on these results, we conclude that membrane complexes comprising TssL and TssM (and probably TssJ) assemble at discrete positions in the cell and are then used to recruit the tail complex subunits. Statistical analyses showed that the number of

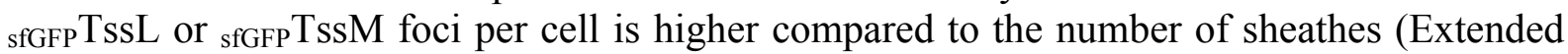
data Fig. 1e) suggesting that the membrane complexes exist in a pre-assembled form. Interestingly, long-term time-lapse recordings showed that these membrane complexes can be re-used for new tail polymerization events (Extended data Fig. 1f). To gain further information on the biogenesis of this initial step, tss $J$ or tssM were deleted in the sfGFPTssL-

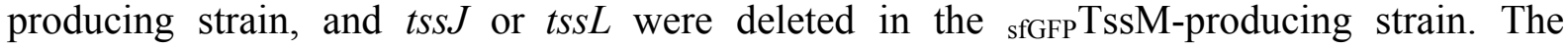
rationale behind these experiments is that if a protein assembled early is missing, the recruitment of late proteins will be affected yielding a diffuse fluorescent signal. Fig. If

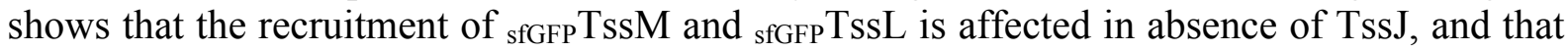

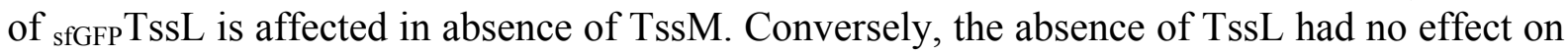
TssM recruitment (Fig. 1f and Extended Fig. 1g). Based on these results, we conclude that TssJ is used as a nucleation factor and that the biogenesis of the T6SS membrane core complex is pursued by the inward sequential addition of TssM and TssL (Fig. 1g).

To gain further insights on the architecture of the T6SS membrane core complex, the $t s s J$, $t s s L$ and $t s s M$ genes were co-expressed in E. coli BL21(DE3). Constructs were designed to add StrepII, FLAG and $6 \times$ His tags at the C-terminus of TssJ, N-terminus of TssL and Nterminus of TssM, respectively (Extended data Fig. 2). Total membranes were isolated and solubilized using detergents. Two-step affinity chromatography followed by gel filtration resulted in the purification of a complex containing TssJ, TssL and TssM (Fig. 2a and Extended data Fig. 2f-h). In this complex, we determined the TssM-TssL stoichiometry as 1 to 1 (Extended data Fig. 2h). Purified complexes were visualized by negative stain electron microscopy (EM) (Fig. 2b and Extended data Fig. 3a). A data set was collected and referencefree classification and averaging revealed characteristic views of the complex (class averages) (Fig. 2b). We observed rocket-shaped and ring-shaped views corresponding to side and end views of the T6SS membrane core complex respectively (Fig. 2b). Rotational symmetry analysis of end view class-averages revealed a clear 5-fold symmetry in the whole TssJLM population (Extended data Fig. 3b). The complex is composed of a base and a tip complex linked by arches (Fig. 2b). The negative stain data set was used to reconstruct a $11.6 \AA$ resolution three-dimensional (3D) volume of the complex with 5-fold symmetry applied (Fig. $2 c$ and Extended data Fig. 3c,d). Local resolution calculations using ResMap ${ }^{30}$ indicated that the local resolution was significantly lower in the base (Extended data Fig. 3e,f). This impaired a correct interpretation of this part of the TssJLM map. Since this could be due to flexibility between the base and the rest of the complex, we performed a local 3D refinement on the base region only, which yielded a 3D reconstruction of the base at $16.6-\AA$ resolution. A composite map where this new reconstruction of the base replaces the equivalent densities in the reconstruction of the whole complex is shown in Fig. 2c. The T6SS membrane core complex is $300 \AA$ in height and $205 \AA$ in diameter (Fig. 2c). It is made of a base that is decorated at its bottom by five hooks and is pierced at its centre by a small hole of $15 \AA$ in diameter (Fig. 2c). Ten arches connect this base to a tip complex of $160 \AA$ in diameter covered by a small cap. Remarkably, five arches gather at the centre of the tip complex to define a 15 to $20-\AA$ diameter channel. The five other arches form a scaffold at the periphery of this complex (Extended data Fig. 4a). Overall, the tip complex is made of internal and external pillars arranged in concentric rings (Extended data Fig. 4a). 
To define how the core complex is inserted in the cell envelope, we first performed differential solubilisation of the inner and outer membranes. The total membrane fraction was solubilized with N-lauryl sarkosyl, a detergent that preferentially solubilize inner membrane proteins. This differential solubilisation resulted in the fractionation of the core complex in both inner and outer membrane fractions (Extended data Fig. 4b), indicating that this complex resides in both membranes. To determine its orientation in the cell envelope, the purified core complex was incubated with anti-StrepII antibodies or Ni-NTA-coated gold particles targeting the TssJ C-terminal StrepII and TssM N-terminal $6 \times$ His tags respectively (Extended data Fig. 4c), prior to EM analyses. Anti-StrepII antibodies labelled the tip complex/cap while the base was labelled by the Ni-NTA gold particles (Extended data Fig. 4c). We concluded that the TssJ C-terminus is located in the tip complex while the TssM N-terminus is located in the base (Extended data Fig. 4c). When the N-terminal cysteine residue of the TssJ lipoprotein was substituted by Ser (C1S) to prevent its lipidation, an intact Tss ${ }^{\mathrm{C} 1 \mathrm{~S}}$-L-M core complex was formed (Extended data Fig. 4b), but differential solubilisation proved the complex mislocalized to the inner membrane fraction only (Extended data Fig. 4b). Hence, TssJ acylation tethers the apex of the complex to the outer membrane whereas the base of the complex is located in the cytoplasm.

We next analysed the EM reconstruction to assign the different regions of the core complex to its components. The volume corresponding to one arch and the corresponding pilar within the tip complex (Extended data Fig. 4a) is comparable in size and shape to that of the isolated TssM periplasmic domain (amino-acids 386-1129; TssMp) in complex with TssJ obtained by Small Angle X-ray Scattering (Extended data Fig. 4d,e). Segmentation of this volume yielded five different sub-volumes (Fig. 3a). We propose that the sub-volume closest to the cap corresponds to TssJ, in agreement with its location close to the outer membrane. The other four sub-volumes would correspond to sub-domains of TssMp. Sub-volume 4 is in close contact with TssJ, suggesting that it corresponds to the C-terminal domain of TssM domain, which was previously shown to mediate contact with $\mathrm{Tss}^{25}$. With sub-volume 3, it forms the tip complex while sub-volumes 1 and 2 correspond to the arches (Fig 3a). Interestingly, the last TssM trans-membrane segment crossing the inner membrane is located just upstream of TssMp. This would place the inner membrane at the bottom of the arches or at the top of the base. The volume of the base $\left(1450 \AA^{3}\right)$ is much bigger than the estimated volume occupied by 10 copies of the cytoplasmic domains of TssM and TssL $\left(825 \AA^{3}\right)$. The crystal structure of TssL cytoplasmic domain dimer ${ }^{28,29}$ could be fitted in the hooks with $88 \%$ correlation (Extended data Fig. 4f). This indicates that the remaining of the base could correspond to the cytoplasmic domain of TssM and the 40 trans-membrane segments bound to detergent (Extended data Fig. 4f).

To gain more insight into the structure of TssMp-TssJ complex, TssMp was produced and purified as described previously ${ }^{25}$. To help crystallization, TssMp complex was subjected to controlled proteolytic digestion ${ }^{31}$. A protease-resistant fragment of an apparent size of $\sim 32$ $\mathrm{kDa}$ (called hereafter $\mathrm{TssM}_{32 \mathrm{Ct}}$; residues 836-1129; Extended data Fig. 5a) was further purified and co-crystallized with $\mathrm{nb} 25$, a specific camelid single-chain nanobody ${ }^{31,32}$. The structure of the TssM $\mathrm{M}_{32 \mathrm{Ct}}$-nb25 complex was solved by molecular replacement using the X-ray structure of nb25 reported previously ${ }^{32}$ (Extended data Fig. 5b; Extended data Table 1). In the complex, the $\mathrm{TssM}_{32 \mathrm{Ct}}$ amino-acid chains are defined in the electron density map between residues 868 and 1107 . We therefore purified a new TssMp fragment (TssM $26 \mathrm{Ct}$ ) encompassing the crystallographic visible chain. This shorter fragment crystallized readily alone as well as in complex with the unacylated TssJ protein (Extended data Table 1). The structure of $\mathrm{Tss}_{26 \mathrm{Ct}}$ is composed of two domains. The $\mathrm{N}$-terminal domain (residues 870-974) 
is a four $\alpha$-helices bundle, covered on one side by a $\beta$-hairpin (Fig. 3b) and on the other side by the C-terminal elongated stretch of the protein. The C-terminal domain (residues 9751085 ) is a 9 -stranded $\beta$-sandwich that contacts nb25 or TssJ (Fig. 3b; Extended Tables 2a,b). This C-terminal domain is followed by a stretch of residues (1086-1107) comprising helix $\alpha 5$ (Fig. 3b). TssJ binds to the apex of the C-terminal domain and the 590- $\AA^{2}$ interaction area involves contacts between TssJ loops L1-2, L3-4 and L5-6 with TssM $26 \mathrm{Ct}$ loops L3-4 and L5-6 (Extended data Fig. 5c, Extended data Table 2b) in agreement with a previous study demonstrating the importance of TssJ loop L1-2 for TssM-TssJ complex formation ${ }^{25}$. Superimposition of the structures of $\mathrm{TssM}_{32 \mathrm{Ct}}-\mathrm{nb} 25$ and $\mathrm{TssM}_{26 \mathrm{Ct}}-\mathrm{Tss} J$ shows that nb25 and TssJ cannot bind simultaneously to TssM (Extended data Fig. 5d), explaining the nb25 in vivo inhibitory effect on T6SS function ${ }^{32}$. The comparison between TssM $26 \mathrm{Ct}^{-}$-TssJ crystal structure and the volume proposed to correspond to TssJ and domain 3 and 4 of TssMp determined by EM resulted in $95 \%$ correlation between the two structures (Fig 3c). This confirms the location of $\mathrm{TssM}_{26 \mathrm{Ct}}-\mathrm{Tss} \mathrm{J}$ in the tip complex (Fig. 3d).

The orientation of TssJ N-terminus places the outer membrane above TssJ, where the cap is located (Fig. 2c, Fig. 4a and Extended data Fig. 5e). Accordingly, close inspection of the proposed oligomeric structure of $\mathrm{TssM}_{26 \mathrm{Ct}}$-TssJ complex could not reveal any obvious trans-membrane region (Extended data Fig. 5f). To test this, we engineered functional cysteine derivatives between the $\beta$-strands of the C-terminal domain of TssM (Extended data Fig. 6a). The extracellular accessibility of these residues was assessed by incubating whole cells with an outer membrane-impermeant cysteine-reactive maleimide. We observed that positions 989, 1005, 1035, 1075 and 1109 were labeled whereas positions 972, 1019, 1062 and 1092 were not (Extended data Fig. 6b and Extended data Table 2c). With the exception of position 1092, all other positions were labeled when cell lysates were used instead of intact cells (Extended data Table 2c). The labeled cysteine substitutions are on the tip of TssM facing the outer membrane (Fig. 4a). Interestingly, residues 989 and 1005 are buried at the interface with TssJ (Extended Data Fig. 6c). Therefore, for being labeled, the TssM-TssJ complex has to dissociate. This result also suggests that the tip of $\mathrm{TssM}_{26 \mathrm{Ct}}$ is exposed to the cell exterior. To test whether TssM stably crosses the outer membrane or accesses the cell exterior temporarily, similar experiments were conducted in a tssBC-deleted background. In absence of the TssB and TssC sheath components, the TssJLM membrane complex is properly assembled but the T6SS is inactive as no sheath assembly or contraction could occur. In $t s s B C$ cells, only position 1109 was labeled (Extended data Fig. 6b and Fig. 4a). These results suggest that TssM $\alpha 5$ helix crosses the outer membrane permanently exposing the $\mathrm{C}$ terminal extension to the extracellular medium whereas part of $\mathrm{TssM}_{26 \mathrm{Ct}}$ domain is exposed transiently at the cell surface during T6SS mechanism of action.

The data presented here allows an unprecedented understanding of the biogenesis, architecture and role of the T6SS TssJLM membrane core complex. This complex anchors the phage tail-like structure to the cell envelope and is thought to serve as conduit to guide the Hcp tube/VgrG spike upon sheath contraction ${ }^{15-17}$. Using fluorescence microscopy, we demonstrate that the three subunits are recruited in a specific order, starting from the outer membrane TssJ lipoprotein and pursued by the sequential addition of TssM and TssL, a hierarchy in agreement with previously published localization and interaction studies ${ }^{17,22-27}$. Therefore, T6SS biogenesis is initiated by an outer membrane lipoprotein nucleation factor and progresses inwards, such as the assembly mechanisms of other bacterial secretion systems ${ }^{33-39}$. Our fluorescence microscopy analyses also showed that the T6SS membrane core complex assembles randomly in the cell envelope, without specific localization. The 
complex is stable and can be used for several events of sheath assembly/contraction, increasing the amount of toxin effectors delivered to the target cell.

The TssJLM complex has a 5-fold symmetry and is composed of 10 copies of each component that assemble a 1.7-MDa structure crossing the inner membrane, the periplasm and anchored to the outer membrane via the TssJ N-terminal lipid moiety. Its architecture is unique compared to other trans-envelope bacterial secretion systems (Extended data Fig. 7a). Based on our accessibility experiments, we propose that upon assembly of other T6SS subunits with the membrane core complex, the TssM C-terminal extension (C-terminal extended stretch following helix $\alpha 5$ in the crystal structure and the remaining 22 non-visible amino-acids) will change its conformation and will cross the outer membrane. The base of the TssJLM complex defines a small cavity and hole that can not accommodate the VgrG protein and potential effectors bound to it (Fig. $4 \mathrm{~b}$ - stages $1 \& 2)^{9,20}$. We propose that the base changes its conformation to receive the baseplate components. This state would correspond to a "resting" state of the T6SS machinery (Fig. 4b - stage 2). Ten arches cross the periplasm and are followed by ten pillars positioned in two concentric layers in the tip complex. The inner pillars define a channel of $15-20 \AA$ in diameter that is not large enough to allow the passage of the $\sim 110 \AA$-large Hcp tube ${ }^{16-18}$ (Extended data Fig. 7b). Interestingly, it was previously shown that TssM undergoes large conformational changes during secretion ${ }^{26}$. Therefore, we propose that the inner TssM pillars are pushed outwards in order to define a wider TssM ring with internal dimensions compatible with the passage of the Hcp tube/VgrG spike (Fig. 4b - stages $3 \& 4$; and Extended data Fig. 7b,c). In other secretion systems, specific components are dedicated to assemble the outer membrane pore. No obvious transmembrane region could be found in the TssM C-terminal domain or in TssJ. It is unlikely that the C-terminal portion of TssM would form a pore of sufficient dimension by itself. Therefore, we propose that the stroke of Hcp-VgrG arrow would mechanically push the Cterminal TssM domain towards the cell exterior, allowing the transient formation of a pore through the outer membrane (Fig. 4b - stage 4). To avoid deleterious effects for the bacterium, one may expect that the C-terminal domain of TssM returns to its initial "resting" conformation at the periplasmic face of the outer membrane once the Hcp tube has been released, closing the outer membrane channel (Fig. $4 b$ - stage 5). Overall, the membrane core complex appears to act like a docking station for the phage-like T66S device. It nucleates the assembly of the rest of the secretion system and then guides the Hcp tube/VgrG spike through the bacterial cell envelope upon sheath contraction. Further studies will be necessary to fully understand the complete assembly process of the T6SS, the trigger that releases sheath contraction and how the Hcp tube/VgrG spike crosses both bacterial and host membranes.

\section{References}

1. West, S. A., Griffin, A. S., \& Gardner, A. Evolutionary explanations for cooperation. Curr Biol. 17, 661-72 (2007).

2. Blango, M. G., \& Mulvey, M. A. Bacterial landlines: contact-dependent signaling in bacterial populations. Curr Opin Microbiol. 12, 177-81 (2009).

3. Russell, A. B., Peterson, S. B., \& Mougous, J. D. Type VI secretion system effectors: poisons with a purpose. Nat Rev Microbiol. 12, 137-48 (2014). 
4. Silverman, J. M., Brunet, Y. R., Cascales, E., \& Mougous, J. D. Structure and regulation of the type VI secretion system. Annu Rev Microbiol. 66, 453-72 (2012).

5. Pukatzki, S., Ma, A. T., Revel, A. T., Sturtevant, D., \& Mekalanos, J. J. Type VI secretion system translocates a phage tail spike-like protein into target cells where it cross-links actin. Proc Natl Acad Sci U S A. 104, 15508-13 (2007).

6. Russell, A. B. et al. Type VI secretion delivers bacteriolytic effectors to target cells. Nature. 475, 343-7 (2011).

7. Russell, A. B. et al. Diverse type VI secretion phospholipases are functionally plastic antibacterial effectors. Nature. 496, 508-12 (2013).

8. Ma, L. S., Hachani, A., Lin, J. S., Filloux, A., \& Lai, E. M. Agrobacterium tumefaciens deploys a superfamily of type VI secretion DNase effectors as weapons for interbacterial competition in planta. Cell Host Microbe. 16, 94-104 (2014).

9. Durand, E., Cambillau, C., Cascales, E., \& Journet, L. VgrG, Tae, Tle, and beyond: the versatile arsenal of Type VI secretion effectors. Trends Microbiol. 22, 498-507 (2014).

10. Borgeaud, S., Metzger, L. C., Scrignari, T., \& Blokesch, M. Bacterial evolution. The type VI secretion system of Vibrio cholerae fosters horizontal gene transfer. Science. 347, 637 (2015).

11. Cascales, E. The type VI secretion toolkit. EMBO Rep. 9, 735-41 (2008).

12. Bönemann, G., Pietrosiuk, A., Diemand, A., Zentgraf, H., \& Mogk, A. Remodelling of VipA/VipB tubules by ClpV-mediated threading is crucial for type VI protein secretion. EMBO J. 28, 315-25 (2009).

13. Basler, M., Pilhofer, M., Henderson, G. P., Jensen, G. J., \& Mekalanos, J. J. Type VI secretion requires a dynamic contractile phage tail-like structure. Nature. 483, 182-6 (2012).

14. Kudryashev, M. et al. Structure of the Type VI secretion system contractile sheath. Cell. 160, 952-62 (2015).

15. Coulthurst, S. J. The Type VI secretion system - a widespread and versatile cell targeting system. Res Microbiol. 164, 640-54 (2013).

16. Ho, B. T., Dong, T. G., \& Mekalanos, J. J. A view to a kill: the bacterial type VI secretion system. Cell Host Microbe. 15, 9-21 (2014).

17. Zoued, A. et al. Architecture and assembly of the Type VI secretion system. Biochim Biophys Acta. 1843, 1664-73 (2014).

18. Bönemann, G., Pietrosiuk, A., \& Mogk, A. Tubules and donuts: a type VI secretion story. Mol Microbiol. 76, 815-21 (2010).

19. Brunet, Y. R., Hénin, J., Celia, H., \& Cascales, E. Type VI secretion and bacteriophage 
tail tubes share a common assembly pathway. EMBO Rep. 15, 315-21 (2014).

20. Shneider, M. M. et al. PAAR-repeat proteins sharpen and diversify the type VI secretion system spike. Nature. 500, 350-3 (2013).

21. Brunet, Y. R., Espinosa, L., Harchouni, S., Mignot, T., \& Cascales, E. Imaging type VI secretion-mediated bacterial killing. Cell Rep. 3, 36-41 (2013).

22. Aschtgen, M. S., Gavioli, M., Dessen, A., Lloubès, R., \& Cascales, E. The SciZ protein anchors the enteroaggregative Escherichia coli Type VI secretion system to the cell wall. Mol Microbiol. 75, 886-99 (2010).

23. Aschtgen, M. S., Bernard, C. S., de Bentzmann, S., Lloubès, R., \& Cascales, E. SciN is an outer membrane lipoprotein required for type VI secretion in enteroaggregative Escherichia coli. J Bacteriol. 190, 7523-31 (2008).

24. Ma, L. S., Lin, J. S., \& Lai, E. M. An IcmF family protein, ImpLM, is an integral inner membrane protein interacting with ImpKL, and its walker a motif is required for type VI secretion system-mediated Hcp secretion in Agrobacterium tumefaciens. J Bacteriol. 191, 4316-29 (2009).

25. Felisberto-Rodrigues, C. et al. Towards a structural comprehension of bacterial type VI secretion systems: characterization of the TssJ-TssM complex of an Escherichia coli pathovar. PLoS Pathog. 7, e1002386 (2011).

26. Ma, L. S., Narberhaus, F., \& Lai, E. M. IcmF family protein TssM exhibits ATPase activity and energizes type VI secretion. J Biol Chem. 287, 15610-21 (2012).

27. Aschtgen, M. S., Zoued, A., Lloubès, R., Journet, L., \& Cascales, E. The C-tail anchored TssL subunit, an essential protein of the enteroaggregative Escherichia coli Sci-1 Type VI secretion system, is inserted by YidC. Microbiologyopen. 1, 71-82 (2012).

28. Durand, E. et al. Structural characterization and oligomerization of the TssL protein, a component shared by bacterial type VI and type IVb secretion systems. J Biol Chem. 287, 14157-68 (2012).

29. Chang, J. H., \& Kim, Y. G. Crystal structure of the bacterial type VI secretion system component TssL from Vibrio cholerae. J. Microbiol. 53, 32-7 (2015).

30. Kucukelbir, A., Sigworth, F. J., \& Tagare, H. D. Quantifying the local resolution of cryoEM density maps. Nat Methods. 11, 63-5 (2014).

31. Nguyen, V. S. et al. Production, crystallization and X-ray diffraction analysis of a complex between a fragment of the TssM T6SS protein and a camelid antibody. Acta Crystallogr F Struct Biol Commun. 71, 266-271 (2015).

32. Nguyen, V. S., Logger, L., et al..Inhibition of Type VI secretion by an anti-TssM llama nanobody. PLOS ONE. in press (2015).

33. Diepold, A. et al. Deciphering the assembly of the Yersinia type III secretion injectisome. 
EMBO J. 29, 1928-40 (2010).

34. Judd, P. K., Kumar, R. B., \& Das, A. Spatial location and requirements for the assembly of the Agrobacterium tumefaciens type IV secretion apparatus. Proc Natl Acad Sci US A. 102, 11498-503 (2005).

35. Hardie, K. R., Lory, S., \& Pugsley, A. P. Insertion of an outer membrane protein in Escherichia coli requires a chaperone-like protein. EMBO J. 15, 978-88 (1996).

36. Drake, S. L., Sandstedt, S. A., \& Koomey, M. PilP, a pilus biogenesis lipoprotein in Neisseria gonorrhoeae, affects expression of PilQ as a high-molecular-mass multimer. Mol Microbiol. 23, 657-68 (1997).

37. Burghout, P. et al. Role of the pilot protein $\mathrm{YscW}$ in the biogenesis of the $\mathrm{YscC}$ secretin in Yersinia enterocolitica. J Bacteriol. 186, 5366-75 (2004).

38. Crago, A. M., \& Koronakis, V. Salmonella InvG forms a ring-like multimer that requires the InvH lipoprotein for outer membrane localization. Mol Microbiol. 30, 47-56 (1998).

39. Daefler, S., \& Russel, M. The Salmonella typhimurium InvH protein is an outer membrane lipoprotein required for the proper localization of InvG. Mol Microbiol. 28, 1367-80 (1998).

Acknowledgements This work was funded by ANR grants ANR-10-JCJC-1303-03 to E.C., Bip:Bip to R.F. ANR-14-CE14-0006-02 to C.C. and E.C. and supported by the French Infrastructure for Integrated Structural Biology (FRISBI) ANR-10-INSB-05-01. E.D. was supported by a post-doctoral fellowship from the Fondation pour la Recherche Médicale (SPF20101221116) and ANR grants Bip:Bip and ANR-10-JCJC-1303-03. V.S.N. is supported by a Ph.D grant from the French Embassy in Vietnam (792803C). A.Z., L.L. and M.S.A were recipients of doctoral fellowships from the French Ministère de la Recherche. A.Z. was recipient of the Fondation pour la Recherche Médicale fellowhip (FDT20140931060). We thank Olivera Francetic for providing anti-DglA and anti-OmpF antibodies. We thank Esther Marza, Petya Violinova Krasteva and Han Remaut for helpful comments on the manuscript, Tâm Mignot, Mathilde Guzzo and Leon Espinosa for advices regarding the fluorescence microscopy experiments and the statistical analyses. We also thank the members of the RF and EC research groups for insightful discussions and suggestions, Roland Lloubès, James Sturgis, Anne Galinier and Yesus-Hans-Hubert Förmm for encouragements.

Author Contributions E.D., A.Z., C.C., E.C. \& R.F. designed the experiments. A.Z. constructed the EAEC mutant and fluorescent strains and performed the fluorescence microscopy experiments and statistical analyses, L.L. and M.S.A. constructed the TssM cysteine derivatives and performed the accessibility experiments. ED assisted by AnD purified the TssJLM complex and performed its biochemical characterization. ED and GPA collected the EM data. ED and RF obtained the 3D reconstruction of the TssJLM complex. VSN, SS, AR \& CC purified, crystallized and solved the X-ray structures. AlD generated the nanobody. BB obtained the energy-minimized models of the closed and open states of the $\mathrm{TssM}_{26 \mathrm{Ct}}$-TssJ complex. 
Structure deposition The EM structure of the TssJLM complex has been deposited in the Electron Microscopy Data Bank under accession number emd-2827. The crystal structures of the TssM ${ }_{32 \mathrm{Ct}}-\mathrm{nb} 25$ complex, and of the TssM ${ }_{26 \mathrm{Ct}}$ fragment and $\mathrm{TssM}_{26 \mathrm{Ct}}-\mathrm{Tss} \mathrm{J}$ complexes have been deposited in the Protein Data Bank under accession numbers 4Y7M, 4Y7L and 4Y7O respectively.

\section{LEGEND TO FIGURES}

Figure 1 | Biogenesis of the T6SS membrane-associated core complex. a. Time-lapse fluorescence microscopy recordings showing localization and dynamics of the ${ }_{\text {sfGFPTssM }}$ and

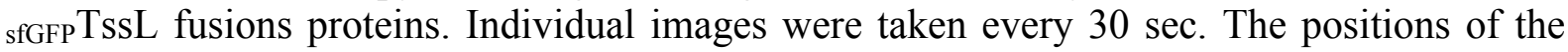
foci are indicated by arrowheads. The scale bars are $1 \mu \mathrm{m}$. Larger fields are presented in

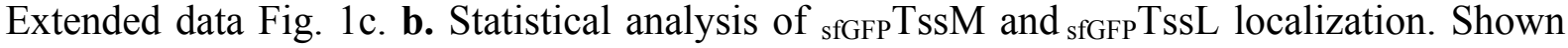

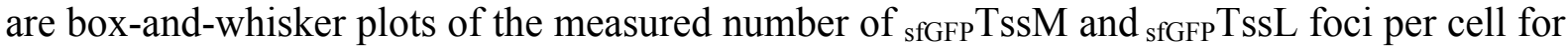
each strain with the lower and upper boundaries of the boxes corresponding to the $25 \%$ and $75 \%$ percentiles respectively. The black bold vertical bar represents the median values for each strain and the whiskers represent the $10 \%$ and $90 \%$ percentiles. Outliers are shown as open circle. $n$ indicates the number of cells studied per strain. c. Spatial repartition of the

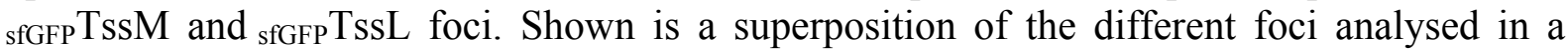
single cell. d. ${ }_{\text {sfGFPTssM }}$ and ${ }_{\mathrm{mch} T s s L}$ proteins co-localize. Fluorescence microscopy recordings showing co-localization between ${ }_{\text {sfGFPTssM }}$ and ${ }_{\mathrm{mCh}} \mathrm{TssL}$ fusion proteins. The positions of the foci are indicated by the arrows. The scale bars are $1 \mu \mathrm{m}$. e. The membrane complex serves as a docking site for tail sheath polymerization. Time-lapse fluorescence microscopy recordings showing co-localization between ${ }_{s f G F P} T s s L$ and $T s s B_{m C h}$ fusion proteins. Individual images were taken every $30 \mathrm{sec}$. Assembly-contraction of the sheath and TssL localization events are schematized in the lower panel. The scale bars are $1 \mu \mathrm{m}$. f. Assembly pathway of the T6SS TssJLM membrane complex. Fluorescence microscopy

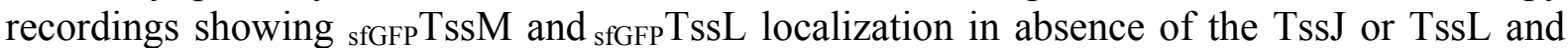
TssJ or TssM proteins respectively. The positions of the foci are indicated by the arrows. The

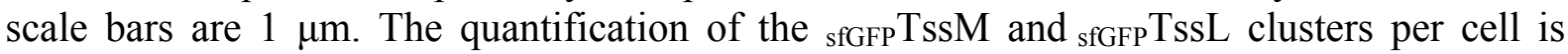
presented in Extended data Fig. 1g. g. Schematic representation of the sequential biogenesis of the T6SS membrane complex. The name of the proteins, their localizations and topologies are shown.

Figure 2 | TssJLM complex purification and structure. a. SDS-PAGE analysis of the purified EAEC TssJLM complex. The bands corresponding to TssM (130 kDa), TssL (24 $\mathrm{kDa})$ and TssJ (18 kDa) after SDS-PAGE and Coomassie blue staining are indicated. b. Representative views (class averages) of purified TssJLM complexes. End to side views are shown from top left to bottom right. The scale bar is $10 \mathrm{~nm}$. c. Structure of the TssJLM complex. Side, cut-away, bottom and top views are shown from top left to bottom right panels respectively. The different regions of the complex are indicated on the cut-away view.

Figure 3 | Structure of the TssJLM tip complex. a. Segmentation of the TssJLM complex reconstruction. Each volume encompassing one arch and the corresponding pillar within the tip complex is segmented in five different domains (shown in different colors). b. Crystal structure of the $\mathrm{TssM}_{26 \mathrm{C}} / \mathrm{Tss}_{\mathrm{sol}}$ complex represented as ribbons. TssJ $\mathrm{sol}_{\mathrm{sol}}$ is colored orange, 
while $\mathrm{TssM}_{26 \mathrm{Ct}}$ is colored cyan ( $\alpha$-domain) and blue ( $\beta$-domain). The $\mathrm{C}$-terminal $\alpha$-helix 5 and the extended stretch are colored magenta. The $\beta$-hairpin $(\beta 1-\beta 2)$ is highlighted in the dashed circle. Two orthogonal views of the crystal structure and its surface representation are shown from left to right (colored as in (a)). c. TssM ${ }_{26 \mathrm{Ct}}$-TssJcrystal structure docked into the EM volume corresponding to TssJ and the TssM periplasmic domains 3 and 4 extracted from both internal and external pillars of the tip complex. d. Energy minimized atomic model of the tip complex structure (left panel, side view; right panel, top view).

Figure 4 | Cell surface accessibility and mechanism of action of the T6SS membrane core complex during secretion. a. Cell surface accessibility studies. Crystal structure of the $\mathrm{TssM}_{26 \mathrm{Ct}}$ represented as ribbons, colored cyan ( $\alpha$-domain) and blue ( $\beta$-domain). The $\mathrm{C}$ terminal $\alpha$-helix 5 and the extended stretch are colored magenta. The $\mathrm{C}$-terminus (lacking in the crystallized fragment) is represented as a random structure beyond the last residue in the crystallographic model. The cysteine substitutions (in spheres representation) used for labelling experiments are positioned in the $\mathrm{TssM}_{26 \mathrm{Ct}}$ crystal structure. Cysteines with extracellular accessibility when the T6SS is active are colored yellow, while the unlabeled ones are colored grey. b. Model of action. The proposed mechanism of action involves 4 sequential stages. 1. The assembled TssJLM complex is not integrally inserted in the outer membrane, but anchored to it by the TssJ N-terminal lipid moiety. This stage corresponds to the electron microscopy reconstruction of the purified TssJLM complex (bottom inset) and the crystal structure of the TssM $\mathrm{M}_{26 \mathrm{Ct}}$-TssJ complex (top inset) presented in this study. 2. Upon assembly of T6SS baseplate and tail components, the C-terminal extremity of TssM inserts into the outer membrane and is therefore accessible at the cell surface (yellow star). This stage corresponds to the "resting" state of the T6SS membrane complex. 3 and 4. The membrane core complex opens to allow the passage of the Hcp tube/VgrG spike or the sheath contraction force induces conformational changes of the TssJLM complex. A molecular model of a $\mathrm{C} 10$ symmetrized $\mathrm{Tss} / \mathrm{TssM} \mathrm{sct}_{26}$ ring is presented (top inset). The apical loops of TssM are exposed at the cell surface (yellow stars). 5. After release of the Hcp tube/VgrG spike, the TssJLM membrane complex complex returns to the resting state, ready to perform another cycle of secretion. 


\section{METHODS}

Strains, media and chemicals - The strains, plasmids and oligonucleotides used in this study are listed in Supplementary Table 1. The E. coli K-12 DH5 $\alpha$ strain was used for cloning steps whereas $E$. coli K-12 BL21(DE3) and T7-Iq pLys strains were used for protein purification. The enteroaggregative E. coli EAEC strain 17-2 was used to engineer gene knock-outs and fusions with fluorescent labels. Strains were routinely grown in LB rich medium (or Terrific broth medium for protein purification) or in Sci-1 inducing medium (SIM; M9 minimal medium, glycerol $0.2 \%$, vitamin B1 $1 \mu \mathrm{g} / \mathrm{mL}$, casaminoacids $100 \mu \mathrm{g} / \mathrm{mL}$, LB $10 \%$, supplemented or not with bactoagar $1.5 \%)^{40}$ with shaking at $37^{\circ}$ C. Plasmids were maintained by the addition of ampicillin $(100 \mu \mathrm{g} / \mathrm{mL}$ for E. coli K$12,200 \mu \mathrm{g} / \mathrm{mL}$ for EAEC) or kanamycin $(50 \mu \mathrm{g} / \mathrm{mL})$. Expression of genes from pETG20A and pRSF vectors was induced with $1 \mathrm{mM}$ of isopropyl- $\beta$-D-thio-galactopyrannoside (IPTG, Eurobio) for 16 hours.

Strain construction - Gene deletion into the enteroaggregative E. coli 17-2 strain was achieved by using a modified one-step inactivation procedure ${ }^{41}$ as previously described ${ }^{23}$ using plasmid $\mathrm{pKOBEG}^{42}$. Briefly, a kanamycin cassette was amplified from plasmid $\mathrm{pKD} 4^{41}$ using oligonucleotide pairs carrying 5' 50-nucleotide extensions homologous to regions adjacent to the gene to be deleted. After electroporation of $600 \mathrm{ng}$ of column-purified PCR product, kanamycin resistant clones were selected and verified by colony-PCR. The kanamycin cassette was then excised using plasmid $\mathrm{pCP} 20^{41}$. Gene deletions were confirmed by colony-PCR. The same procedure was used to introduce the $m C h e r r y$ - or $s f G F P$-coding sequences downstream the start codon (vector pKD4-sfGFP or pKD4$m$ Cherry) or the $m$ Cherry-coding sequence upstream the stop codon (vector pmCherry-KD4). This procedure yields strains producing fusion proteins from their original chromosomal loci.

Plasmid construction - Polymerase Chain Reactions (PCR) were performed using the Phusion DNA polymerase (Thermo scientific). Restriction enzymes were purchased from New England Biolabs and used according to the manufacturer's instructions. Custom oligonucleotides were synthesized by Sigma Aldrich and are listed in Supplementary Table 1. Enteroaggregative E. coli 17-2 chromosomal DNA was used as a template for all PCRs. E. coli strain DH5 $\alpha$ was used for cloning procedures. The pETG20A vector derivative encoding the periplasmic domain of the TssM periplasmic domain (TssMp, residues 386 to 1129 ) or the TssJ soluble domain have been previously described ${ }^{25}$. The fragment encoding $\mathrm{TssM}_{26 \mathrm{Ct}}$ (residues 869-1107) was cloned into the $\mathrm{pETG20A}$ vector by restrictionfree cloning ${ }^{43}$. The pRSF-Tss $J^{\mathrm{S}}$ intermediate plasmid was constructed by restriction cloning. Briefly, the sequence encoding the full-length tsS $J$ gene (residues 1-178) was PCR-amplified using primers RSF-sJ-F and RSF-sJ-R. The PCR introduced a 5' NdeI and a 3' XhoI restriction sites and a Cterminal Streptavidin extension. The tss $J$ PCR product was sub-cloned into the pRSF-Duet (Novagen) MCS2 corresponding restriction sites. The pRSF-Tss $J^{\mathrm{S}}-{ }_{-} \mathrm{L}-{ }^{\mathrm{H}} \mathrm{M}$ (encoding C-terminally StrepII-tagged TssJ, N-terminally FLAG-tagged TssL and N-terminally $6 \times$ His-tagged TssM) has been constructed by restriction-free cloning ${ }^{43}$ as previously described ${ }^{22}$. Briefly, the sequence encoding the full-length $t s s L$ (residues 1-217) and full-length tssM (residues 1-1129) genes were PCR-amplified using the primer pairs RSF-fL-F/RSF-fL-R and RSF-hM-F/RSF-hM-R, respectively. The two PCR products (tssL and $t s s M$ ) were synthesized with 30-bp overhangs, from both $5^{\prime}$ and $3^{\prime}$ ends, corresponding to the designed integration sites into the pRSF-Tss $\mathrm{J}^{\mathrm{S}}$ plasmid. The double-stranded product of the first PCR has then been used as oligonucleotides for a second PCR using the target vector as template. The introduction of the C1S mutation in TssJ was performed by Quick-change mutagenesis of the pRSFTss ${ }^{\mathrm{S}}-{ }_{-}{ }^{2}-{ }^{H} \mathrm{M}$ plasmid using oligonucleotides Jcs-F and Jcs-R. Plasmid pIBA37-TssM was constructed by restriction-free cloning and cysteine derivatives were obtained by Quick-change mutagenesis using the TssM-C757S mutant.

Hep release assay. Cells producing FLAG- or HA-tagged Hcp from plasmids pUCHcp FLAG $_{\text {or }}$ pOKHсp ${ }_{\mathrm{HA}}{ }^{22,23}$ were grown in SIM to an $\mathrm{OD}_{600} \sim 0.8$. Supernatant and cell fractions were separated as previously described ${ }^{23}$. Briefly, $2 \times 10^{9}$ cells were harvested and collected by centrifugation at $2,000 \times \mathrm{g}$ for $5 \mathrm{~min}$. The supernatant fraction was then subjected to a second low-speed centrifugation and then 
at $16,000 \times g$ for $15 \mathrm{~min}$. The supernatant was filtered on sterile polyester membranes with a pore size of $0.2 \mu \mathrm{m}$ (membrex 25 PET, membraPure $\mathrm{GmbH}$ ) before overnight precipitation with trichloroacetic acid $15 \%$ on ice. Cells and precipitated supernatants were resuspended in loading buffer and analyzed by Sodium Dodecyl Sulfate-Polyacrylamide Gel Electrophoresis (SDS-PAGE) and immunoblotting with the anti-FLAG or anti-HA antibody. As control for cell lysis, Western blots were probed with antibodies raised against the periplasmic TolB protein. The assays have been performed from three independent cultures, and a representative experiment is shown.

Interbacterial competition assay. The antibacterial growth competition assay was performed as described for the studies on the Citrobacter rodentium and EAEC Sci-2 T6SSs ${ }^{21,44}$ with modifications. The wild-type E. coli strain W3110 bearing the $\mathrm{Kan}^{\mathrm{R}}$ pUA66-rrnB plasmid $^{45}$ was used as prey in the competition assay. Attacker and prey cells were grown for 16 hours in LB medium, then diluted in SIM to allow maximal expression of the $s c i-1$ gene cluster ${ }^{40}$. Once the culture reached an $\mathrm{OD}_{600 \mathrm{~nm}} \sim$ 0.8 , the cells were harvested and normalized to an $\mathrm{OD}_{600 \mathrm{~nm}}$ of 0.5 in SIM. Attacker and prey cells were mixed to a 4:1 ratio and 20- $\mu 1$ drops of the mixture were spotted in triplicate onto a pre-warmed dry SIM agar plate supplemented or not with anhydrotetracyclin $0.02 \mu \mathrm{g} / \mathrm{ml}$. After overnight incubation at $37^{\circ} \mathrm{C}$, the bacterial spots were then cut off, and cells were resuspended in SIM to an $\mathrm{OD}_{600 \mathrm{~nm}}$ of 0.5 . $200 \mu 1$ of serial dilutions were plated on kanamycin LB plates and the number of colonies was scored after overnight incubation at $37^{\circ} \mathrm{C}$. The assays have been performed at least from three independent cultures, with technical triplicates and a representative technical triplicate is shown.

Fluorescence microscopy, image treatment and statistical analyses - Fluorescence microscopy experiments have been performed essentially as described ${ }^{21,46}$. Briefly, cells were grown overnight in $\mathrm{LB}$ medium and diluted to an $\mathrm{OD}_{600 \mathrm{~nm}} \sim 0.04$ into Sci-1 inducing medium (SIM). Exponentially growing cells $\left(\mathrm{OD}_{600 \mathrm{~nm}} \sim 0.8-1\right)$ were harvested, washed in phosphate buffered saline buffer (PBS), resuspended in PBS to an $\mathrm{OD}_{600 \mathrm{~nm}} \sim 50$, spotted on a thin pad of $1.5 \%$ agarose in PBS, covered with a cover slip and incubated for one hour at $37^{\circ} \mathrm{C}$ prior to microscopy acquisition. For each experiment, ten independent fields were manually defined with a motorized stage (Prior Scientific) and stored (X, $\mathrm{Y}, \mathrm{Z}, \mathrm{PFS}$-offset) in our custom automation system designed for time-lapse experiments. Fluorescence and phase contrast micrographs were captured every $30 \mathrm{sec}$. using an automated and inverted epifluorescence microscope TE2000-E-PFS (Nikon, France) equipped with Perfect Focus System (PFS). PFS automatically maintains focus so that the point of interest within a specimen is always kept in sharp focus at all times despite mechanical or thermal perturbations. Images were recorded with a CoolSNAP HQ 2 (Roper Scientific, Roper Scientific SARL, France) and a 100×/1.4 DLL objective. The excitation light was emitted by a $120 \mathrm{~W}$ metal halide light. All fluorescence images were acquired with a minimal exposure time to minimize bleaching and phototoxicity effects. The sfGFP images were recorded by using the ET-GFP filter set (Chroma 49002) using an exposure time of 200-400ms. The mCherry images were recorded by using the ET-mCherry filter set (Chroma 49008) using an exposure time of $100-200 \mathrm{~ms}$. Slight movements of the whole field during the time of the experiment were corrected by registering individual frames using StackReg and Image Stabilizer plugins for ImageJ. sfGFP and mCherry fluorescence channels were adjusted and merged using ImageJ (http://rsb.info.nih.gov/ij/). sfGFP fluorescence sets of data were treated to monitor foci detection. Noise and background were reduced using the 'Subtract Background' (20 pixels Rolling Ball) plugin from Fiji (Image J/NIH). The sfGFP foci were automatically detected by a simple image processing: (1) create a mask of cell surface and dilate (2) detect the individual cells using the "Analyse particle" plugin of Fiji (3) identify foci by the "Find Maxima" process in Fiji. To avoid false positive, each event was manually controlled in the original data. Microscopy analyses have been performed at least six times, each in technical triplicate and a representative experiment is shown. Box and whisker representing the number of detected foci for each strain were made with $\mathrm{R}$ software. T-tests were performed on $\mathrm{R}$ to statistically compare each population. Sub-pixel resolution tracking of fluorescent foci: Fluorescent foci were detected using a local and sub-pixel resolution maxima detection algorithm and tracked over time with a specifically developed plug-in for ImageJ. The X and Y coordinates were obtained for each fluorescent focus on each frame. The Mean Square Displacement (MSD) was 
calculated as the distance of the foci from its location at t0 at each time using $\mathrm{R}$ software and plotted over time. For each strain tested, the MSD of at least 10 individual focus trajectories was calculated.

Inner and outer membrane separation - Cells were broken using an Emulsiflex-C5 (Avestin) and the crude membrane fraction was isolated by ultracentrifugation at $100,000 \times g$ for $45 \mathrm{~min}$. Outer and inner membranes were separated by differential solubilization. Inner membranes were solubilized by $0.5 \%$ sodium N-lauroyl sarcosyl (SLS) in $50 \mathrm{mM}$ Tris-HCl pH8.0 for 30 minutes at room temperature. The insoluble material containing the outer membrane fraction was isolated by ultracentrifugation at $100,000 \times \mathrm{g}$ for $20 \mathrm{~min}$. The outer membrane pellet was then solubilized in SDS-loading buffer. The assay has been performed in triplicate, from three independent cultures and a representative experiment is shown.

TssJLM complex production and purification - The pRSF-Tss ${ }^{\mathrm{S}}-{ }^{\mathrm{F}} \mathrm{L}-{ }^{\mathrm{H}} \mathrm{M}$ plasmid was transformed into the E. coli BL21(DE3) expression strain (Invitrogen). Cells were grown at $37^{\circ} \mathrm{C}$ in lysogeny broth (LB) to an $\mathrm{OD}_{600} \sim 0.7$ and the expression of the tssJLM genes was induced with $1.0 \mathrm{mM}$ IPTG for 16 hours at $16^{\circ} \mathrm{C}$. Cell pellets were resuspended in ice-cold $50 \mathrm{mM}$ Tris- $\mathrm{HCl} \mathrm{pH} 8.0,50 \mathrm{mM} \mathrm{NaCl}, 1 \mathrm{mM}$ EDTA and $1 \mathrm{mM}$ TCEP, supplemented with $100 \mu \mathrm{g} / \mathrm{ml}$ of DNase I, $100 \mu \mathrm{g} / \mathrm{ml}$ of lysozyme and EDTA-free protease inhibitor (Roche). After sonication, $\mathrm{MgCl}_{2}$ was added to the final concentration of $10 \mathrm{mM}$ and the cell suspension was further broken using an Emulsiflex-C5 (Avestin). The broken cell suspension was clarified by centrifugation at $38,500 \times \mathrm{g}$ for $20 \mathrm{~min}$. The membrane fraction was then collected by centrifugation at $98,000 \times \mathrm{g}$ for $45 \mathrm{~min}$. Membranes were mechanically homogenized and solubilized in $50 \mathrm{mM}$ Tris- $\mathrm{HCl} \mathrm{pH} 8.0,50 \mathrm{mM} \mathrm{NaCl}, 0.5 \% \mathrm{w} / \mathrm{v}$ n-dodecyl- $\beta$-D-maltopyranoside (DDM, Anatrace), 0.75\% w/v Decyl Maltose Neopentyl Glycol (DM-NPG, Anatrace), 0.5\% w/v digitonin (Sigma-Aldrich), $100 \mu \mathrm{M}$ TCEP and $1 \mathrm{mM}$ EDTA at $22^{\circ} \mathrm{C}$ for $45 \mathrm{~min}$. The suspension was clarified by centrifugation at $98,000 \times \mathrm{g}$ for $20 \mathrm{~min}$. The supernatant was loaded onto a 5 -ml StrepTrap $\mathrm{HP}$ (GE Healthcare) column and then washed with $50 \mathrm{mM}$ Tris- $\mathrm{HCl} \mathrm{pH} \mathrm{8.0,50} \mathrm{mM} \mathrm{NaCl,} 0.05 \% \mathrm{w} / \mathrm{v}$ DM-NPG (Affinity buffer) at $4{ }^{\circ} \mathrm{C}$. The TssJLM core complex was eluted in the affinity buffer supplemented with $2.5 \mathrm{mM}$ desthiobiotin (IBA) into a 5-ml HisTrap HP (GE Healthcare) column. The column was then washed in the affinity buffer supplemented with $20 \mathrm{mM}$ imidazole and the TssJLM core complex was eluted in the same buffer supplemented with $500 \mathrm{mM}$ imidazole. Peak fractions were pooled and loaded onto a Superose $610 / 300$ column (GE Healthcare) equilibrated in $50 \mathrm{mM}$ Tris- $\mathrm{HCl} \mathrm{pH} 8.0,50 \mathrm{mM} \mathrm{NaCl}, 0.025 \% \mathrm{w} / \mathrm{v}$ DM-NPG. The TssJLM core complex eluted as a single monodisperse peak close to the void volume of the column. The sample was used immediately for electron microscopy sample preparation.

Stoichiometry analyses - The purified TssJLM core membrane complex was diluted to a final concentration of $0.1 \mathrm{mg} / \mathrm{mL}$ and denaturated at $100^{\circ} \mathrm{C}$ for 10 minutes after the addition of $1 \%$ dosium dodecyl-sulphate. The denatured sample was incubated in presence of $40 \mu \mathrm{M}$ of Alexa Fluor ${ }^{\circledR} 532$ C5-maleimide (Invitrogen) and $1 \mathrm{mM}$ TCEP (Pierce) for $2 \mathrm{~h}$ at room temperature. The labeled proteins were separated by SDS-PAGE and protein-bound fluorescence was visualized and quantified using a Fujifilm FLA-3000 scanner. The assay has been performed in triplicate, from three independent TssJLM complex preparations, and a representative experiment is shown. The quantification is expressed with the standard deviation from the three biological replicates.

Electron microscopy and image processing - Determination of the TssJLM core membrane complex structure was achieved by negative-stain electron microscopy. Nine microlitres of suitably diluted $(\sim 0.01 \mathrm{mg} / \mathrm{ml})$ TssJLM complex sample was spotted to glow-discharged carbon-coated copper grids (Agar Scientific). After $30 \mathrm{sec}$ of absorption, the sample was blotted, washed with three drops of water and then stained with $2 \%$ uranyl acetate. Images were recorded automatically using the EPU software on a FEG microscope operating at a voltage of $200 \mathrm{kV}$ and a defocus range of $0.6-25 \mathrm{~nm}$, using a FEI Falcon-II detector (Gatan) at a nominal magnification of 50,000, yielding a pixel size of $1.9 \AA$. A dose rate of 25 electrons per $\AA^{2}$ per second, and an exposure time of $1 \mathrm{sec}$. were used. A total of 72,146 particles were automatically selected from 1,200 independent images and extracted within boxes of 280 pixels $\times 280$ pixels using EMAN2/BOXER ${ }^{47}$. The CTF was estimated and corrected by 
phase flipping using EMAN2 (e2ctf). All two- and three-dimensional (2D and 3D) classifications and refinements were performed using RELION $1.3^{48,49}$. We used three rounds of reference-free 2D class averaging to clean up the automatically selected dataset. Only highly populated classes displaying high-resolution features were conserved during this procedure and a final dataset of 26,544 particles was assembled. An initial 3D-model was generated in EMAN2 using using 30 classes. 3D classification was then performed in Relion with 5 classes. The particles corresponding to most populated class $(\sim 16,738)$ were used for refinement. Relion auto-refine procedure was used to obtain a final reconstruction at $11.56-\AA \AA$ resolution after masking and with C5 symmetry imposed. Reported resolutions are based on the gold-standard Fourier shell correlation (FSC) 0.143 criterion, and FSC curve were corrected for the effects of a soft mask on the FSC curve using high-resolution noise substitution $^{50}$. Before visualization, all density maps were corrected for the modulation transfer function of the detector and then sharpened by applying an ad-hoc negative B-factor (-1000).. Local resolution variations were estimated using ResMap ${ }^{51}$.

3D maps display and analysis - Three-dimensional reconstructions were displayed and rendered in USCF Chimera segmented using the SEGGER module implemented in UCSF Chimera ${ }^{52}$. Segments corresponding to individual structural domains are represented in Fig. 3c-e. All other maps were left un-segmented. A volume/mass conversion of $0.81 \mathrm{Da} \AA^{-3}$ was used to estimate the volume occupied by TssM and TssL cytoplasmic domains.

Protein production and purification for SAXS and X-ray analyses - The periplasmic domain of the TssM protein, TssMp (residues 386 to 1129) was produced and purified as described previously ${ }^{25}$. The purified recombinant TssMp was digested with trypsin (at a 1000:1 molecular ratio) at room temperature for 24 hours. The reaction was quenched by the addition of $1 \mathrm{mM}$ phenyl-methanesulfonyl fluoride (PMSF) and the insoluble TssMp fragments were discarded by centrifugation at $20,000 \times g$ for 30 minutes. A proteolysis-resistant fragment of an apparent size of $\sim 32 \mathrm{kDa}$ (called hereafter $\mathrm{TssM}_{32 \mathrm{Ct}}$ ) was further purified by consecutive ion exchange (Mono Q 5/50 GL column, GE Healthcare) and size exclusion (Superdex 75 16/600 HL column) chromatographies using a Äkta system (GE-Healthcare). The purified fragment was subjected to N-terminal Edman sequencing. A PVDF membrane was rinsed three times with a water/ethanol mixture (10/90) and inserted in the A cartridge of a Procise 494A sequencer. After five cycles of Edman degradation, the sequence DYGSL was identified by mass spectrometry, indicating that cleavage after Arg-834 generated a C-terminal fragment of theoretical mass $32,398 \mathrm{Da}$, in agreement with the 32-kDa band observed by SDS-PAGE analyses.

For production and purification of the $\mathrm{TssM}_{26 \mathrm{Ct}}$ fragment (Thr-869 to Glu-1107), E. coli BL21(DE3) cells grown in the TB medium carrying plasmid pETG20A-TssM $26 \mathrm{Ct}$ were grown to an $\mathrm{OD}_{600 \mathrm{~nm}} \sim 0.6$ and the expression of $\mathrm{TssM}_{26 \mathrm{Ct}}$ was induced by the addition of $0.5 \mathrm{mM}$ IPTG for 16 hours at $17^{\circ} \mathrm{C}$. Cells were collected by centrifugation at $10,000 \times g$ at $4{ }^{\circ} \mathrm{C}$ for $15 \mathrm{~min}$. The cell pellet was resuspended in lysis buffer and lysed by sonication. The lysate was clarified by centrifugation at $20,000 \times \mathrm{g}$ at $4{ }^{\circ} \mathrm{C}$ for $15 \mathrm{~min}$, and the supernatant containing the Trx-His 6 -tev- $\mathrm{TssM}_{26 \mathrm{ct}}$ fusion protein was purified by consecutive $\mathrm{Ni}^{2+}-$ affinity and size exclusion (Superdex 75 column) chromatographies on a Äkta purifier (GE Healthcare). The fractions containing the protein of interest were pooled and the $6 \times$ His-tagged TEV protease was added $(5 \% \mathrm{w} / \mathrm{w})$. The cleaved protein was purified using $\mathrm{Ni}^{2+}$ affinity, removing the Trx$\mathrm{His}_{6}$, followed by size exclusion chromatography (Superdex 75 column) on a Äkta purifier (GE Healthcare). Over $100 \mathrm{mg}$ of $\mathrm{TssM}_{26 \mathrm{ct}}$ fragment were obtained per litre of culture. The purified protein was verified by mass spectrometry, before being concentrated up to $8.7 \mathrm{mg} / \mathrm{ml}$ in $20 \mathrm{mM}$ Tris- $\mathrm{HCl} \mathrm{pH}$ 8.0, $\mathrm{NaCl} 150 \mathrm{mM}$.

The production of nanobody nb25 and the formation of its complex with $\mathrm{TssM}_{32 \mathrm{Ct}}$ have been described previously $^{31,32}$. The production of unacylated TssJ was previously described ${ }^{25}$. The $\mathrm{TssM}_{26 \mathrm{Ct}} \mathrm{Tss}^{\mathrm{T} J}$ complex was obtained by mixing $\mathrm{TssM}_{26 \mathrm{Ct}}(8.7 \mathrm{mg} / \mathrm{ml})$ with purified TssJ $(30 \mathrm{mg} / \mathrm{ml})$ in a $1: 1.2$ molecular ratio and the complex was then concentrated up to $15 \mathrm{mg} / \mathrm{ml}$ using centricon (cut-off of $10,000 \mathrm{Da}$ ) in $20 \mathrm{mM}$ Tris- $\mathrm{HCl} \mathrm{pH} 8.0,150 \mathrm{mM} \mathrm{NaCl}$.

Small-Angle X-ray Scattering analysis and $a b$ initio 3D shape reconstruction - Small-Angle X-ray Scattering (SAXS) analyses were performed at the ID29 beamline (European Synchrotron Radiation 
Facility, Grenoble, France) at a working energy of $12.5 \mathrm{keV}(\lambda=0.931 \AA)$. Thirty microlitres of protein solution at $1.6 \mathrm{mg} / \mathrm{mL}$ in Tris- $\mathrm{HCl} 20 \mathrm{mM} \mathrm{pH} 8.0, \mathrm{NaCl} 150 \mathrm{mM}$ were loaded by a robotic system into a 2-mm quartz capillary mounted in a vacuum and ten independent 10 -sec exposures were collected on a Pilatus $6 \mathrm{M}-\mathrm{F}$ detector placed at a distance of $2.85 \mathrm{~m}$ for each protein concentration. Individual frames were processed automatically and independently at the beamline by the data collection software (BsxCUBE), yielding radially averaged normalized intensities as a function of the momentum transfer $\mathrm{q}$, with $\mathrm{q}=4 \pi \sin (\theta) / \lambda$, where $2 \theta$ is the total scattering angle and $\lambda$ is the X-ray wavelength. Data were collected in the range $\mathrm{q}=0.04-6 \mathrm{~nm}^{-1}$. The ten frames were combined to give the average scattering curve for each measurement. Data points affected by aggregation, possibly induced by radiation damage, were excluded. Scattering from the buffer alone was also measured prior to and after each sample analysis and the average of these two buffer measures was used for background subtraction using the program PRIMUS ${ }^{53}$ from the ATSAS package ${ }^{54}$. PRIMUS was also used to perform Guinier analysis ${ }^{55}$ of the low q data, which provides an estimate of the radius of gyration $\left(\mathrm{R}_{g}\right)$. Regularized indirect transforms of the scattering data were carried out with the program $\mathrm{GNOM}^{56}$ to obtain $\mathrm{P}(r)$ functions of interatomic distances. The $\mathrm{P}(r)$ function has a maximum at the most probable intermolecular distance and goes to zero at Dmax, the maximum intramolecular distance. The values of Dmax were chosen to fit with the experimental data and to have a positive $\mathrm{P}(r)$ function. Tridimensionnal (3D) bead models that fit with the scattering data were built with the program DAMMIF ${ }^{57}$. Twenty independent DAMMIF runs were performed using the scattering profile of the TRX-His-TssJp and TRX-His-TssMp complex, with data extending up to $0.35 \mathrm{~nm}^{-1}$, using slow mode settings, assuming no symmetry and allowing for a maximum 500 steps to grant convergence. The models resulting from independent runs were superimposed using the DAMAVER suit ${ }^{58}$. This yielded an initial alignment of structures based on their axes of inertia followed by minimisation of the normalized spatial discrepancy (NSD) ${ }^{59}$. The NSD was therefore computed between a set of twenty independent reconstructions, with a range of NSD from 0.678 to 0.815 . The aligned structures were then averaged, giving an effective occupancy to each voxel in the model, and filtered at half-maximal occupancy to produce models of the appropriate volume that were used for all subsequent analyses. All the models were similar in terms of agreement with the experimental data, as measured by DAMMIF $\chi$ parameter and the quality of the fit to the experimental curve. The DAMFILT average volume was used as the final model of the TRX-His-TssJ and TRX-His-TssMp complex.

NanoGold-Ni-TNA labelling. The TssJLM complex was spotted onto a glow-discharged carbon coated grid (CF-400, Electron Microscopy Sciences). After $1 \mathrm{~min}$, excess liquid was blotted, and the grid was washed on a drop of cold purification buffer $(50 \mathrm{mM}$ Tris $\mathrm{pH} 8,50 \mathrm{mM} \mathrm{NaCl}, 0.025 \% \mathrm{w} / \mathrm{v}$ DM-NPG) containing $50 \mathrm{mM}$ imidazole, quickly blotted and deposited on a second drop of the same buffer in the presence of $5 \mathrm{nM}$ nanogold-Ni-NTA beads (Nanoprobes). After 2 min, the grid was rinsed sequentially for $20 \mathrm{sec}$ with one drop of purification buffer, one drop of the same buffer without detergent and three drops of $2 \%$ uranyl acetate. Images were collected on an FEI Tecnai F20 FEG microscope operating at a voltage of $200 \mathrm{kV}$, equipped with a direct electron detector (Falcon II). Particles were selected manually using EMAN2. The assay has been performed at least in triplicate, from independent TssJLM complex preparations and representative particles are shown.

Anti-strep labelling. The TssJLM complex was mixed with monoclonal anti-Strep antibodies (Sigma) at a TssJLM complex:antibody ratio of $2: 1$. The mixture was incubated at $4{ }^{\circ} \mathrm{C}$ for 30 minutes and the labelled complex was isolated by gel filtration. The sample was analysed by negative stain EM as described above for negative stain EM of the unlabelled TssJLM complex. The assay has been performed at least in triplicate, from independent TssJLM complex preparations and representative particles are shown.

Crystallisation and structure determination - The crystallization of the $\mathrm{TssM}_{32 \mathrm{Ct}} \mathrm{nb} 25$ has been described previously ${ }^{31}$. For $\mathrm{TssM}_{26 \mathrm{Ct}}$ alone several kits were used for crystallization screening, including STURA, WIZARD, MDL, INDEX, and PEGs. A hit was observed in the PEGs kit, within a well containing $0.2 \mathrm{M}$ Zinc acetate and $20 \%(\mathrm{~m} / \mathrm{v})$ PEG3350. Crystal optimization was performed by varying PEG3350 amount in the $15-25 \%$ range in $0.1 \mathrm{M}$ Sodium acetate and $0.2 \mathrm{M} \mathrm{ZnCl}_{2}$ at $\mathrm{pH}$ 
varying between 3.8 and 5.5. Crystals appeared after few days in 20\% PEG3350, $0.1 \mathrm{M}$ sodium acetate $\mathrm{pH} 4$ and $0.2 \mathrm{M} \mathrm{ZnCl}_{2}$. Crystals were tested at ESRF beamline ID23-1 after cryo-cooling in the crystallization liquor supplemented with $12.5 \%$ propylene glycol.

The TssM $26 \mathrm{Ct}^{-}$TssJ complex was screened for crystallization using the PEGs and PACT1 kits. Hits were observed in PACT1. All contained $\mathrm{Zn}^{2+}: 0.01 \mathrm{M}$ Zinc chloride, $0.1 \mathrm{M}$ Sodium acetate $\mathrm{pH} 5,20 \%$ (w/v) PEG 6000; or 0.01 M Zinc chloride, 0.1 M MES pH 6, 20\% (w/v) PEG 6000. Crystal optimization was performed by using PEG6000 in the $10-20 \%$ range in $0.1 \mathrm{M}$ sodium acetate/MES pH 4.75-6, and $\mathrm{ZnCl}_{2}$ at $0,0.01,0.05$ and $0.2 \mathrm{M}$. No crystals were obtained in conditions without $\mathrm{ZnCl}_{2}$ or containing $0.2 \mathrm{M} \mathrm{ZnCl}_{2}$. By contrast, well shaped crystals appeared in $50 \mathrm{mM} \mathrm{ZnCl}, 15 \%$ PEG6000 and 0.1 sodium acetate $\mathrm{pH} 4.75$. Crystals were cryo-cooled with polypropylene glycol $12.5 \%$ but diffracted to only $\sim 4.0 \AA$ at the Soleil Proxima 2 beamline. Further crystal involved crystallization in LIMBRO plates. Large crystals were obtained by mixing $6 \mu$ of protein and $2 \mu 1$ of well solution in 50 $\mathrm{mM} \mathrm{ZnCl} 2,15 \%$ PEG6000, $94 \mathrm{mM}$ sodium acetate $\mathrm{pH} 4.75$ and $6 \mathrm{mM}$ MES pH6. Crystals were dipped in polypropylene glycol for $\sim 20 \mathrm{sec}$ and exposed at the ESRF beamline ID23-1.

Data collection was performed at $100 \mathrm{~K}$ at beamline Proxima 1 (Soleil synchrotron, Saint Aubin, France) for $\mathrm{TssM}_{32 \mathrm{Ct}} \mathrm{nb} 25$ and at beamline ID23-1 (ESRF synchrotron, Grenoble, France) for $\mathrm{TssM}_{26 \mathrm{Ct}}$ alone and for the $\mathrm{TssM}_{26 \mathrm{Ct}}-\mathrm{TssJ}$ complex. Data were processed by the $\mathrm{XDS}^{60}$ package and scaled with XSCALE (Extended data Table 1).

The structure of the $\mathrm{Tss}_{32 \mathrm{Ct}} \mathrm{nb} 25$ complex was determined by molecular replacement with Molrep ${ }^{61}$ using the previously determined structure of nb25 (PDB 4QGY) ${ }^{32}$. After refining the positions of the two nb25 molecules in the asymmetric unit by rigid body refinement with AutoBuster ${ }^{62}$, an electron density map was calculated at $1.92-\AA$ resolution. Features such as a-helices were easily identified, making it possible to trace manually the model of $\mathrm{TssM}_{32 \mathrm{Ct}}$ using $\mathrm{COOT}^{63}$, alternated with cycles of refinement with AutoBuster ${ }^{62}$, with non-crystallographic symmetry (n.c.s.) restraints and TLS groups refinement ${ }^{64}$, features used in all refinement procedures described below. The final structure at 1.92- $\AA$ resolution has $\mathrm{R}_{\text {work }} / \mathrm{R}_{\text {free }}$ values of $18.4 / 21.0 \%, 96.3 \%$ of the residues in the preferred area of the Ramachandran plot and no outliers (Extended data Table 1).

The structure of $\mathrm{TssM}_{26 \mathrm{Ct}}$ alone was solved by molecular replacement with Molrep ${ }^{61}$ using the refined model of $\mathrm{TssM}_{32 \mathrm{ct}}$ from the $\mathrm{TssM}_{32 \mathrm{Ct}} \mathrm{nb} 25$ complex. The initial structure model was improved through iterative refinement with AutoBuster ${ }^{62}$ and manual refitting with COOT $^{63}$. The final structure at 1.51$\AA$ resolution has $\mathrm{R}_{\text {work }} / \mathrm{R}_{\text {free }}$ values of $19.2 / 20.2 \%, 97.4 \%$ of the residues in the preferred area of the Ramachandran plot and no outliers (Extended data Table 1).

The structure of the $\mathrm{TssM}_{26 \mathrm{Ct}}$-TssJ complex was solved by molecular replacement with Molrep ${ }^{61}$ using the refined structure of TssM ${ }_{26 \mathrm{ct}}$ and the previously determined TssJ structure (PDB 3RX9) ${ }^{25}$ in which all the alternate conformations were removed. A first round of rigid body refinement and four cycles of Phenix ${ }^{65}$ cartesian simulated annealing were performed. The resulting model was improved through iterative refinement with AutoBuster ${ }^{62}$ and manual refitting with $\mathrm{COOT}^{63}$. The final structure at 2.24- $\AA$ resolution has $\mathrm{R}_{\text {work }} / \mathrm{R}_{\text {free }}$ values of $20.0 / 22.3 \%, 96.9 \%$ of the residues in the preferred area of the Ramachandran plot, and four outliers residues in very poorly defined loops (Extended data Table 1). The $\mathrm{TssM}_{32 \mathrm{Ct}}-\mathrm{nb} 25, \mathrm{TssM}_{26 \mathrm{Ct}}$ and $\mathrm{TssM}_{26 \mathrm{Ct}}-\mathrm{Tss} \mathrm{J}$ structures form similar homodimers in the asymmetric unit. However, as reported by PISA ${ }^{66}$, and the known topologies of TssM and Tss ${ }^{23,24}$, these dimers are not biologically relevant. Molecular contacts were analysed by the PISA server ${ }^{33}$ and figures were prepared with Chimera ${ }^{52}$ and Pymol $^{67}$.

The crystal structures of the TssM $\mathrm{M}_{32 \mathrm{Ct}}-\mathrm{nb} 25$ complex, and of the $\mathrm{TssM}_{26 \mathrm{Ct}}$ fragment and $\mathrm{TssM}_{26 \mathrm{Ct}}$-TssJ complexes have been deposited in the Protein Data Bank under accession numbers PDB-4Y7M, PDB$4 \mathrm{Y} 7 \mathrm{~L}$ and PDB-4Y7O respectively.

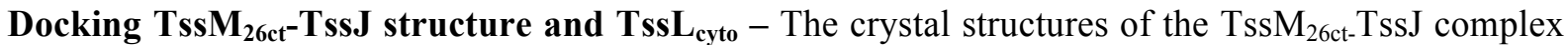
and of the TssL cytoplasmic domain (PDB $3 \mathrm{U} 66)^{28}$ were docked automatically using Chimera ${ }^{52}$ after map segmentation.

Refinement of docked $\mathbf{T s s M}_{26 \mathrm{Ct}}-\mathrm{J}$ pentamer in the $\mathbf{E M}$ density map - The atomic model of the docked $\mathrm{Tss}_{26 \mathrm{Ct}} \mathrm{J}$ structure was refined in the EM density with RSRef ${ }^{68}$. First, missing side-chain and polar hydrogen atoms were added with Modeller ${ }^{69}$. The structure was minimized using 2000 steps of least-squares conjugate gradients refinement in presence of distance restraints for hydrogen bonds and 
backbone dihedral angle restraints to maintain secondary structures. The minimization was performed with the real-space objective function calculated by RSRef in $\mathrm{CNS}^{70}$. The $\mathrm{C}_{5}$ symmetry was enforced by strict NCS restraints. The total energy included internal parameters (bond length, bond angle, improper and dihedrals) and non-bonded interactions with full Van der Waals and electrostatic potentials using a $7.5 \AA$ cutoff. The final correlation coefficient between the EM reconstruction and refined $\mathrm{TssM}_{26 \mathrm{Ct}^{-}} \mathrm{J}$ atomic model is 0.929 (as calculated by RSRef), whereas it was 0.706 before minimization.

Modeling of TssM $\mathrm{M}_{26 \mathrm{Ct}}-\mathrm{J}$ decamer - The atomic position of a $\mathrm{TssM}_{26 \mathrm{Ct}} \mathrm{J}$ protomer from the outer ring of the pentamer served as starting structure to generate a $\mathrm{TssM}_{26 \mathrm{Ct}} \mathrm{-J}$ decamer model with cyclic 10 fold symmetry using $\mathrm{CNS}^{70}$. The symmetry was enforced by NCS strict restraints (rotations of 36 degrees around the symmetry axis). First, 5000 steps of rigid body minimization were performed including only inter-protomer energetic contributions (full Van der Waals and electrostatics). After a short all-atom minimization (300 steps), $1.5 \mathrm{ps}$ of molecular dynamics (MD) simulation at 1000K was performed, followed by 300 steps of minimization and 10ps of MD simulation at 200K. Minimizations and MD simulations were realized with both intra-protomer and inter-protomer energetic contributions activated and the backbone conformation of the protomer was restrained with harmonic constraints.

Substituted Cysteine Accessibility Method (SCAM) - Cysteine accessibility experiments were carried out on whole cells, mainly as described ${ }^{71,72}$ with modifications. A 20 -ml culture of wild-type or $\triangle t s s B C$ strains producing a periplasmic cysteine-less TssM (Cys727-to-Ser) or derivatives bearing cysteine substitutions were induced for $t s s M$ gene expression with $0.05 \mu \mathrm{g} / \mathrm{ml}$ anhydrotetracyclin (AHT) for 1 hour. Cells were harvested, resuspended in buffer A (100 mM Hepes (pH 7.5), $150 \mathrm{mM}$ $\mathrm{NaCl}, 25 \mathrm{mM} \mathrm{MgCl}_{2}$ ) to a final $\mathrm{OD}_{600}$ of 12 in $500 \mathrm{ml}$ of buffer A. Bovine Serum Albumin (BSA)coupled maleimide (Sigma-Aldrich) was added to a final concentration of $100 \mu \mathrm{M}$ (from a $20 \mathrm{mM}$ stock freshly dissolved in DMSO) and the cells were incubated for $30 \mathrm{~min}$. at $25^{\circ} \mathrm{C}$. $\beta$ Mercaptoethanol (20 mM final concentration) was added to quench the biotinylation reaction, and cells were washed twice in buffer A, and resuspended in buffer A containing $N$-ethyl maleimide (final concentration $5 \mathrm{mM}$ ) to block all free sulfhydryl residues. After incubation $20 \mathrm{~min}$ at $25^{\circ} \mathrm{C}$, cells were disrupted by sonication. Membranes recovered by ultracentifugation at $100,000 \times \mathrm{g}$ for $40 \mathrm{~min}$. were resuspended in Laemmli buffer prior to SDS-PAGE analysis and immunodetection with anti-FLAG antibodies (to detect the TssM proteins). Controls were performed by labelling total membranes from the same samples instead of whole cells. The assay has been performed in triplicate, from three independent cultures and representative experiment is shown.

SDS-PAGE, protein transfer, immunostaining and antibodies - SDS-Polyacrylamide gel electrophoresis was performed on Biorad mini-protean systems using standard protocols. For immunostaining, proteins were transferred onto $0.2 \mu \mathrm{m}$ nitrocellulose membranes (Amersham Protran), and immunoblots were probed with primary antibodies and goat secondary antibodies coupled to alkaline phosphatase, and developed in alkaline buffer in presence of 5-bromo-4-chloro-3indolylphosphate and nitroblue tetrazolium. The anti-TolB is from our laboratory collection, while the anti-HA (3F10 clone, Roche), anti-FLAG (M2 clone, Sigma Aldrich), anti-StrepII (Sigma Aldrich), anti-5his (Sigma Aldrich) monoclonal antibodies and alkaline phosphatase-conjugated goat antirabbit, or mouse secondary antibodies (Millipore) have been purchased as indicated.

\section{REFERENCES}

40. Brunet, Y. R., Bernard, C. S., Gavioli, M., Lloubès, R., \& Cascales, E. An epigenetic switch involving overlapping fur and DNA methylation optimizes expression of a type VI secretion gene cluster. PLoS Genet. 7, e1002205 (2011).

41. Datsenko, K. A., \& Wanner, B. L. One-step inactivation of chromosomal genes in Escherichia 
coli K-12 using PCR products. Proc Natl Acad Sci U S A. 97, 6640-5 (2000).

42. Chaveroche, M. K., Ghigo, J. M., \& d'Enfert, C. A rapid method for efficient gene replacement in the filamentous fungus Aspergillus nidulans. Nucleic Acids Res. 28, 97 (2000).

43. van den Ent, F., \& Löwe, J. RF cloning: a restriction-free method for inserting target genes into plasmids. J Biochem Biophys Methods. 67, 67-74 (2006).

44. Gueguen, E., \& Cascales, E. Promoter swapping unveils the role of the Citrobacter rodentium CTS1 type VI secretion system in interbacterial competition. Appl Environ Microbiol. 79, 32-8 (2013).

45. Zaslaver, A., et al. A comprehensive library of fluorescent transcriptional reporters for Escherichia coli. Nat Methods. 3, 623-8 (2006).

46. Zoued, A., et al. TssK is a trimeric cytoplasmic protein interacting with components of both phage-like and membrane anchoring complexes of the type VI secretion system. J Biol Chem. 288, 27031-41 (2013).

47. Tang, G., et al. EMAN2: an extensible image processing suite for electron microscopy, Journal of structural biology, 157, 38-46 (2007).

48. Scheres, S. H. RELION: implementation of a Bayesian approach to cryo-EM structure determination. J Struct Biol. 180, 519-30 (2012).

49. Scheres, S. H. Semi-automated selection of cryo-EM particles in RELION-1.3. J Struct Biol. 189, 114-22 (2015).

50. Chen, S., et al. High-resolution noise substitution to measure overfitting and validate resolution in 3D structure determination by single particle electron cryomicroscopy. Ultramicroscopy. 135, 24-35 (2013).

51. Kucukelbir, A., Sigworth, F. J., \& Tagare, H. D. Quantifying the local resolution of cryo-EM density maps. Nat Methods. 11, 63-5 (2014).

52. Pettersen, E. F., et al. UCSF Chimera - a visualization system for exploratory research and analysis. J Comput Chem. 25, 1605-12 (2004).

53. Konarev, P.V., Volkov, V.V., Sokolova, A.V., Koch, M.H., \& Svergun, D.I. PRIMUS: a Windows PC-based system for small-angle scattering data analysis $J$ Appl Cryst 36, 1277-1282 (2003).

54. Konarev, P.V., Petoukhov, M.V., Volkov, V.V., \& Svergun, D.I. ATSAS 2.1, a program package for small-angle scattering data analysis J Appl Cryst 39, 277-286 (2006).

55. Guinier, A. La diffraction des rayons $\mathrm{X}$ aux très petits angles; application à l'étude de phénomènes ultramicroscopiques. Ann Phys (Paris) 12, 161-237 (1939).

56. Svergun, D.I. Determination of the regularization parameter in indirect-transform methods using perceptual criteria. J Appl Crystallogr 25, 495-503 (1992).

57. Franke, D., \& Svergun, D.I. DAMMIF, a program for rapid ab-initio shape determination in small-angle scattering J Appl Crystallogr 42, 342-346 (2009). 
58. Volkov, V.V., \& Svergun, D.I. Uniqueness of $a b$ initio shape determination in small-angle scattering J Appl Crystallogr 36, 860-864 (2003).

59. Kozin, M.B., \& Svergun, D.I. Automated matching of high- and low-resolution structural models J Appl Crystallogr 34, 33-41 (2001).

60. Kabsch, W. XDS. Acta Crystallogr D Biol Crystallogr 66, 125-132 (2010).

61. Vagin, A., \& Teplyakov, A. Molecular replacement with MOLREP. Acta Crystallogr D Biol Crystallogr 66, 22-25 (2010).

62. Blanc, E., et al. Refinement of severely incomplete structures with maximum likelihood in BUSTER-TNT. Acta Crystallogr D Biol Crystallogr 60, 2210-2221 (2004).

63. Emsley, P., Lohkamp, B., Scott, W. G., \& Cowtan, K. Features and development of Coot. Acta Crystallogr D Biol Crystallogr 66, 486-501 (2010).

64. Winn, M. D., Murshudov, G. N., \& Papiz, M. Z. Macromolecular TLS refinement in REFMAC at moderate resolutions. Methods Enzymol 374, 300-321 (2003).

65. Adams, P. D., et al. PHENIX: a comprehensive Python-based system for macromolecular structure solution. Acta Crystallogr D Biol Crystallogr 66, 213-221 (2010).

66. Krissinel, E., \& Henrick, K. Inference of macromolecular assemblies from crystalline state. $J$ Mol Biol 372, 774-797 (2007).

67. The PyMOL Molecular Graphics System, Version 1.5.0.4 Schrödinger, LLC.

68. Chapman, M. S., Trzynka, A., \& Chapman, B. K. Atomic modeling of cryo-electron microscopy reconstructions - Joint refinement of model and imaging parameters. J Struct Biol 182, 10-21 (2013).

69. Sali, A., \& Blundell, T. L.. Comparative protein modelling by satisfaction of spatial restraints. $J$ Mol Biol 234, 779-815 (1993).

70. Brunger, A. T.. Version 1.2 of the Crystallography and NMR system. Nat Protocols 2, 27282733 (2007).

71. Bogdanov, M., Zhang, W., Xie, J., \& Dowhan, W. Transmembrane protein topology mapping by the substituted cysteine accessibility method (SCAM(TM)): application to lipid-specific membrane protein topogenesis. Methods. 36, 148-71 (2005).

72. Goemaere, E. L., Devert, A., Lloubès, R., \& Cascales, E. Movements of the TolR C-terminal domain depend on TolQR ionizable key residues and regulate activity of the Tol complex. $J$ Biol Chem. 282, 17749-57 (2007). 


\section{LEGEND TO EXTENDED DATA TABLES}

Extended Data Table 1 : Data collection and refinement statistics

$\S$ Eeach data set has been collected on a unique crystal

*Highest resolution shell is shown in parenthesis.

Extended Data Table 2 : Interactions and accesibility data.

Table 2a. Interactions between TssM and nb25

Table 2b Interactions between TssM and TssJ.

Distances are indicated in $\AA$. The letter $\mathrm{H}$ indicates that atoms establish a hydrogen bond

Table 2c. TssM cysteine accessibility.

WAS: Water accessible surface of the original amino-acids (measured in the unbound TssM / in the TssM-TssJ complex) * not visible in the electron density map

\section{LEGEND TO EXTENDED DATA FIGURES}

\section{Extended data Figure 1}

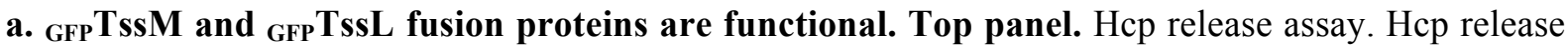
was assessed by separating whole cells $(\mathrm{C})$ and supernatant $(\mathrm{SN})$ fractions from the indicated strains. A total of $1 \times 10^{9}$ cells and the TCA-precipitated material from the supernatant of $2 \times 10^{9}$ cells were analysed by Western blot using anti-FLAG monoclonal antibody (lower panel) and anti-TolB polyclonal antibodies as a lysis control (upper panel). The molecular weight markers (in $\mathrm{kDa}$ ) are indicated on the left. Bottom panel. Anti-bacterial assay. The anti-bacterial activity was assessed by mixing kanamycin-resistant prey E. coli K-12 cells with the indicated attacker cells for 16 hours at $37^{\circ} \mathrm{C}$ in sci-1-inducing medium (SIM). The number of recovered E. coli prey cells is indicated in the lower graph (in log of colony-forming units (cfu)). The circles indicate values from three independent assays, and the average is indicated by the bar. b. Tss $\mathrm{J}_{\mathrm{sfGFP}}$ and $T s \mathrm{~J}_{\mathrm{mCh}}$ fusion proteins are nonfunctional. Hcp release was assessed by separating whole cells (C) and supernatant (SN) fractions from the indicated strains. A total of $1 \times 10^{9}$ cells and the TCA-precipitated material from the supernatant of $2 \times 10^{9}$ cells were analysed by Western blot using anti-FLAG monoclonal antibody (lower panel) and anti-TolB polyclonal antibodies as a lysis control (upper panel). The molecular

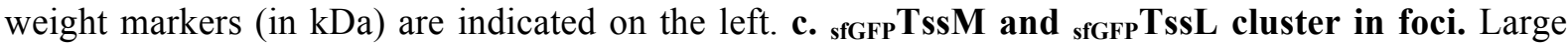

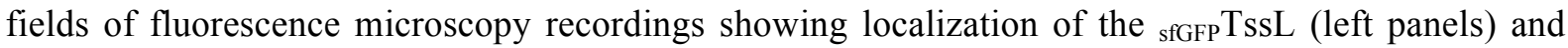
sfGFPTssM (right panels) fusions proteins. The positions of selected foci are indicated by arrowheads.

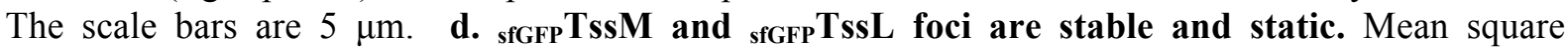

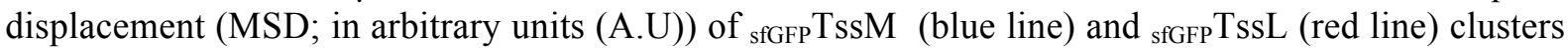
were measured by sub-pixel tracking of fluorescent foci and plotted over time (in min). e. The TssBC sheath tubular structures assemble on TssJLM membrane complexes. Statistical analyses reporting the average number of sheath per cell compared to the number of membrane complexes per cell, highlighting the observation that the number of membrane complexes is $\geq$ to the number of sheathes. Lower and upper boundaries of the boxes correspond to the $25 \%$ and $75 \%$ percentiles respectively. The black bold horizontal bar represents the median values for each strain and the whiskers represent the $10 \%$ and $90 \%$ percentiles. $\mathrm{n}$ indicates the number of cells studied per strain. $\mathbf{f}$. Long-term fluorescence microscopy recordings. Time-lapse fluorescence microscopy recordings showing localization and dynamics of the ${ }_{\mathrm{sfGFP}} \mathrm{TssL}$ and $\mathrm{TssB}_{\mathrm{mCherry}}$ fusions proteins. Individual images were taken every $15 \mathrm{~min}$. Assembly-contraction of the sheath and TssL localization events are schematized in the lower panel. The scale bars are $1 \mu \mathrm{m}$. g. Statistical analysis of ${ }_{\text {sfGFPTssM }}$ and sfGFPTssL localization in various tss backgrounds. Shown are box-and-whisker plots of the measured

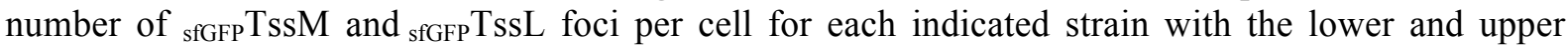
boundaries of the boxes corresponding to the $25 \%$ and $75 \%$ percentiles respectively. The black bold horizontal bar represents the median values for each strain and the whiskers represent the $10 \%$ and $90 \%$ percentiles. Outliers are shown as open circle. $n$ indicates the number of cells studied per strain. 


\section{Extended data Figure 2}

a-e. T6SS operon genomic organization and constructs used for in vitro analyses. a. Schematic representation of the T6SS sci- 1 gene cluster from entero-aggregative Escherichia coli. The numbers on top refer to the gene locus tag (EC042_XXXX). Genes encoding core components (identified by their names on bottom, e.g., " $B$ " refers to the $t s s B$ gene) are colored grey. Genes of unknown function are colored white. The three genes used to reconstitute the core membrane complex are colored orange $(t s s J)$, blue $(t s s L)$ and green $(t s s M)$. b. Schematic representation of the engineered constructs: the $t s s J$, $t s s L$ and $t s s M$ genes were amplified with an additional Shine Dalgarno (SD) sequence and 3' StrepII, 5' FLAG and 5' $6 \times$ His tags respectively. These three fragments were cloned into the pRSF-Duet vector (c). This construct allows the production of the C-terminally StrepII-tagged TssJ outer membrane $(\mathrm{OM})$ lipoprotein and N-terminally FLAG-tagged TssL and $6 \times$ His-tagged TssM inner membrane (IM) proteins (d, e). The proteins are schematized and their boundaries and principal characteristics (TM, trans-membrane segments; SP, signal peptide; CYS, acylated cysteine) are indicated (d) and their topologies shown in e. The additional TssM constructs (TssMp, TssM $32 \mathrm{Ct}$ and $\mathrm{TssM}_{26 \mathrm{Ct}}$ ) used for SAXS or X-ray analyses are shown on bottom.

f-h. Purification and biochemical characterization of the T6SS membrane core complex. f. Analytical size exclusion chromatography analysis of the purified TssJLM complex (continuous line) on a Superose 6 column, calibrated with 75-, 158-, 440- and 660-kDa molecular weight markers (dotted lines). The molecular weight of each marker (in $\mathrm{kDa}$ ) is indicated on the top of the corresponding peak. An arrow indicates the position of the peak fraction corresponding to the TssJLM complex. g. SDS-PAGE of the purified TssJLM complex analyzed by Coomassie staining (C.B.) or immunoblotting using anti-His ( $\alpha$-His); -FLAG ( $\alpha$-FLAG) and -StrepII ( $\alpha$-STREP) antibodies. h. Left panel, Cysteine labelling of the purified TssJLM complex in reducing and denaturing conditions as described in Materials and Methods. The total number of cysteine residues is 9 for TssM, 5 for TssL and 0 for TssJ (the N-terminal cysteine is acylated). Right panel, plot showing the relative amount of TssL compared to TssM (densitometry relative to the number of free cysteine residues, fixed at 1 for TssM).

\section{Extended data Figure 3}

a. Negative stain electron microscopy of the EAEC TssJLM complex. a. Representative micrograph of the dataset used for image processing. Isolated TssJLM complex were clearly visible (white circles). b. Plot of the rotational autocorrelation function for a representative class average of an end-view. c. Fourier Shell Correlation (FSC) curve of the TssJLM reconstruction. The Gold standard FSC curve was calculated in Relion using the masked reconstruction of the TssJLM complex. The resolution at 0.143 correlation was $11.56 \AA$. d. Top panel Side and corresponding cut-away view of the 3D reconstruction for the whole TssJLM complex Bottom panel. Local resolution as calculated by Resmap. The TssJLM volume (left reconstruction, side view; right reconstruction, cut-away view) is colored according to the local resolution from high resolution $(\sim 12 \AA)$ in blue to low resolution (> $30 \AA$ ) in red. e. Fourier Shell Correlation (FSC) curve of the TssJLM base. The Gold standard FSC curve was calculated in Relion using the unmasked reconstruction of the TssJLM base. The resolution at 0.143 correlation was $16.6 \AA$. f. Top, side and bottom views of the 3D reconstruction after specific refinement of the base.

\section{Extended data Figure 4}

a. Segmentation of the TssJLM reconstruction. Left panel. Above the base, ten equivalent densities could be defined by segmentation. They are arranged in two concentric rings. The internal ring is represented in green in the top panel and the external ring is represented in blue in the bottom panel. Right panel . Cut-out views of the complex showing the arrangement of the two concentric rings at different levels (grey lines) along the periplasmic portion of the TssJLM complex. The cut-out views 
are seen from the bottom of the complex. b. Requirement of TssJ lipidation for complex assembly and insertion into the outer membrane. Left panel Membrane fractionation by differential solubilization followed by immunoblot analysis. Total membrane extracts from cells producing the wild-type (WT) TssJLM complex or the TssJLM complex with an unacylated variant of TssJ (Cys1to-Ser substitution, CS) were solubilized by Lauroyl sarcosine (SLS) to separate inner membranes

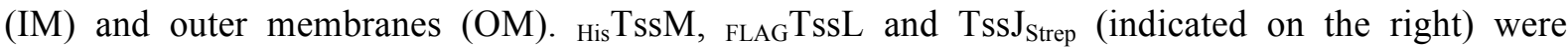
revealed by anti-His, anti-FLAG and anti-StrepII antibodies respectively. Controls include immunodetection of the inner membrane DglA diacylglycrol lipase and the outer membrane OmpF porin. Wild-type TssJLM complex co-fractionates with both the inner and outer membrane fractions whereas the CS mutated complex co-fractionates only with the inner membrane fraction. top right panel. Negative stain Electron Microscopy of the mutated Tss $\mathrm{C}_{\mathrm{CS}} \mathrm{LM}$ complex. Representative micrograph of the dataset used for image processing. Isolated $\mathrm{Tss}_{\mathrm{CS}} \mathrm{LM}$ complex were clearly visible (white circles). Bottom right panel. Gallery of representative class averages generated after reference free 2D classification in Relion. End to side views are shown from top left to bottom right. c. Orientation of the TssJLM complex in the cell envelope. Left panel. Schematic representation of the TssJ (J, orange), TssL (L, blue) and TssM (M, green) proteins. Their localization, main characteritics (lipidation or trans-membrane segments shown in black) and the location of the $6 \times$ His and StrepII tags (red balls) are indicated. The strepII and $6 \times \mathrm{His}$ tags were introduced at the $\mathrm{C}$-terminus and $\mathrm{N}$-terminus of TssJ and TssM respectively. Middle panel. Immune and Nanogold labelling coupled to electron microscopy. Anti-StrepII or Nanogold-NTA were incubated with the TssJLM complex and visualized by negative stain electron microscopy. A gallery of representative views is presented (Top row, StrepII labelling; bottom row, Ni-NTA labelling). The white arrowheads point the StrepII antibody (a schematic diagram with the StrepII antibody depicted as blue circle is shown on top). Right panel. The position of the StrepII antibody (targeting TssJ C-terminus) and of the Ni-NTA gold particle (targeting TssM N-terminus) are indicated on the TssJLM reconstruction. d. Small-angle X-ray scattering data and low-resolution structure of Tss $M_{p}$-TssJ complex. Top left. Experimental scattering data (green crosses) and the fitting curve (continuous red line) calculated from an ab initio model of the TssM $\mathrm{p}_{\mathrm{p}}$-TssJ complex. Top right. Guinier plot (dots) with the linear fit (continuous line). Bottom left. Distance distribution function of the TssM $\mathrm{p}_{\mathrm{p}}$-TssJ complex. Bottom right. SAXS envelope (grey surface) of the "best representative" model of the TssM $\mathrm{p}_{\mathrm{p}}$-TssJ complex. Each view is rotated by $90^{\circ}$ around the Y-axis. e. Location of the TssMp-TssJ complex SAXS envelope in the 3D reconstruction of TssJLM complex. Left panel. The volume of the $\mathrm{TssM}_{\mathrm{p}}-\mathrm{Tss} \mathrm{J}$ complex determined by SAXS was docked into the EM 3D reconstruction of the TssJLM complex (top panel). Two optimal docking positions were found with both $82 \%$ correlation with the EM map (colored magenta and pink). The corresponding volumes in the EM map were extracted (bottom panel). They correspond to the same volume displayed in Extended data Fig. 4a. Right panel. Direct comparison of the SAXS (magenta) and EM (blue and green) volumes corresponding to the $\mathrm{TssM}_{\mathrm{p}}-\mathrm{Tss} \mathrm{J}$ complex. The volumes are equivalent in size and shape. f. TssL cytoplasmic docking into the TssJLM complex base. Fitting of the TssL cytoplasmic domain $\left(\mathrm{TssL}_{\mathrm{cyto}}\right)^{1}$ dimer in green ribbons in the hooks found in the base. Top and bottom panels correspond to side and bottom views respectively.

\section{Extended data Figure 5}

a.Amino-acids sequence of TssM. The different domains as well as the fragments used in this study are indicated (yellow, trans-membrane helix; grey, cytoplasmic domain; green, blue and purple, periplasmic domain; blue and purple, C-terminal domain corresponding to the $\mathrm{TssM}_{32 \mathrm{Ct}}$ fragment; purple, C-terminal domain corresponding to the $\mathrm{TssM}_{26 \mathrm{Ct}}$ fragment). b. Crystal structure of the

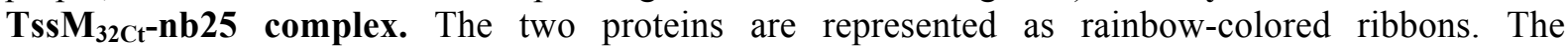
complementary determining regions (CDRs 1-3, colored blue, green and red, respectively) of nb25 are indicated. The inset highlights the $\mathrm{TssM}_{32 \mathrm{Ct}}-\mathrm{nb} 25$ interface: the $\mathrm{TssM}_{32 \mathrm{Ct}}$ surface is colored beige whereas nb25 is represented as rainbow-colored ribbons; the side chains of the amino-acids in contact with $\mathrm{TssM}_{32 \mathrm{Ct}}$ are indicated. The nb25 nanobody binds TssM C-terminal domain, and covers a surface area of $580 \AA^{2}$ by inserting its protruding CDR3 between TssM 32 Ct loops L5-6 and L9-10. The contacts between the two proteins are listed in Extended data Table 2a. c. Crystal structure of the TssM $\mathbf{M}_{26 \mathbf{C t}^{-}}$ TssJ complex. Left panel. The two proteins are represented as ribbons and colored in rainbow mode. 
Middle panel. Same view rotated by $90^{\circ}$. The TssJ loop 1-2, previously shown to contact $\operatorname{TssM}^{2}$ is indicated. Right panel. TssM ${ }_{26 \mathrm{Ct}}$ TssJ interface. Top panel: the $\mathrm{TssM}_{26 \mathrm{Ct}}$ surface is colored violet, whereas TssJ is represented as rainbow-colored ribbons. The TssJ side-chains of the amino-acids in contact with TssM are indicated. The loops are numbered according to the flanking $\beta$-strands. Bottom panel: the TssJ surface is colored beige whereas $\mathrm{TssM}_{26 \mathrm{Ct}}$ is represented as rainbow-colored ribbons. The TssM side-chains of the amino-acids in contact with TssJ are indicated. The contacts between the two proteins are listed in Extended data Table 2b. d. Comparison of the binding sites of $\mathbf{n b 2 5}$ and TssJ on TssM. Left panel. The structure of the TssM $26 \mathrm{Ct}^{-} \mathrm{Tss} J$ complex (rainbow colored) has been superimposed to the structure of the $\mathrm{TssM}_{32 \mathrm{Ct}} \mathrm{nb} 25$ complex (only nb25 is shown in grey for clarity). Right panel. The same partners as in the left panel in surface representation. TssM $\mathrm{M}_{26 \mathrm{Ct}}$ (violet), TssJ (green) and nb25 (pink). e. Insertion of the TssJ lipid anchor in the outer membrane. Left panel. TssJ structure ${ }^{2}$ with the N-terminal 24 residues (absent in the crystal structure). This $\mathrm{N}$-terminal extension (in magenta), predicted to be disordered, was modelled in Chimera using Modeller. The first cysteine residue is acylated to allow anchorage to the inner leaflet of the outer membrane (orange rectangle). Right panel. Docking of the $\mathrm{TssM}_{26 \mathrm{Ct}}$ TssJ complex in the electron microscopy $3 \mathrm{D}$ reconstruction of the TssJLM complex (only the uppermost - tip - part of the TssJLM complex is shown). Left panel: two TssM ${ }_{26 \mathrm{Ct}}{ }^{-T s s} \mathrm{~J}$ were docked into the inner and outer pillar of the tip complex. Right panel: docking in each pillar of the TssJLM tip complex (C5 symmetry). f. Hydrophobicity of the TssM $\mathbf{M}_{26 \mathrm{C}}$-TssJ complex. Surface representation of the $\mathrm{TssM}_{26 \mathrm{Ct}}{ }^{-T s s} J$ decamer (left panel, top view; right panel, side view). The hydrophobicity of the surface residues is displayed (blue to red scale from most hydrophilic to most hydrophobic). No obvious hydrophobic patch is visible at the surface of the complex.

\section{Extended data Figure 6}

a. Functionality of the TssM cysteine variants. Hcp release was assessed by separating whole cells (C) and supernatant (SN) fractions from the wild-type (WT) 17-2 strain and its $\Delta t s s M$ derivative producing a wild-type allele of TssM or TssM cysteine substitution derivatives (as indicated). A total of $1 \times 10^{9}$ cells and the TCA-precipitated material from the supernatant of $2 \times 10^{9}$ cells were analysed by Western blot using anti-HA monoclonal antibody (lower panel) and anti-TolB polyclonal antibodies as a lysis control (upper panel). The molecular weight markers (in $\mathrm{kDa}$ ) are indicated on the left. $\mathbf{b}$. Cysteine substitution labeling. Accessibility to cysteine residues positioned in TssM domain 4 loops was assessed by treating isolated membranes (M) or whole cells (WC) of the indicated strain (WT, wild-type 17-2; $\Delta t s s B C ; \Delta t s s J$ ) producing the indicated TssM cysteine derivative (in red letters) with the cysteine-reactive, membrane-impermeant Bovine Serum Albumin (BSA)-maleimide (BSA-mal). Samples corresponding to a total of $5 \times 10^{9}$ cells were analysed by Western blot using anti-FLAG monoclonal antibody. The position of the TssM protein $(\sim 125-\mathrm{kDa})$ is indicated as wall as that of a retarded band corresponding to BSA-maleimide-coupled TssM $(\sim 190-\mathrm{kDa} ; *)$. The molecular weight markers (in kDa) are indicated. c. Close-up on the Tss $\mathbf{M}_{26 \mathrm{Ct}}$-Tss $\mathbf{J}$ interface. $\mathrm{TssM}_{26 \mathrm{Ct}}$ is represented in blue ribbons. TssJ is represented in orange ribbons and orange transparent surface. TssM residues accessible from the cell exterior when the T6SS is functional are indicated by yellow spheres whereas un-accessible residues are shown by grey spheres. The accessible residues 989 and 1005 are buried at the interface between TssM and TssJ, suggesting that this interface is likely disrupted during T6SS assembly and/or function. Left and right panels are orthogonal views of the same molecule.

\section{Extended data Figure 7}

a. Comparison between the T6SS TssJLM membrane core complex structure and other bacterial secretion systems. From left to right, the E. coli AcrAB-TolC multidrug efflux pump (emd5915) ${ }^{3}$, the EAEC T6SS membrane core complex (this study, emd-2827), the Shigella T3SS trans-

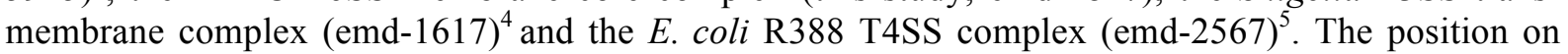
the inner membrane (IM) and outer membrane (OM) are indicated (C, cytoplasm; $\mathrm{P}$, periplasm). The scale bar is $10 \mathrm{~nm}$. b. Docking of the Hcp tube/VgrG spike into the TssJLM 3D reconstruction. Left panel. Before sheath contraction. The Hcp tube/VgrG spike (VgrG in yellow and Hcp in green. 
surface representation) was manually docked in the 3D reconstruction of TssJLM complex (grey surface). The diameter of the channel defined by the closed tip complex is not large enough to allow the passage of the tube/spike, suggesting that large conformational changes likely occur. The cavity at the tip of VgrG could be filled by VgrG-bound PAAR modules or toxin effectors ${ }^{6}$. Right panel. During sheath contraction. The diameter of the $\mathrm{C} 10$ symmetrized $\mathrm{TssM}_{26 \mathrm{Ct}}$ model (represented as ribbons) is compatible with the passage of the Hcp tube/VgrG spike (same colors as in the left panel). c. Closed and open forms of the $\mathbf{T s s}_{26 \mathrm{Ct}}$ oligomer. Crystal structure of $\mathrm{TssM}_{26 \mathrm{Ct}}$ represented as ribbons and transparent surface. The $\mathrm{TssM}_{26 \mathrm{Ct}} \alpha$ - and $\beta$-domains are colored cyan and blue, respectively. The C-terminal $\alpha$-helix 5 and the extended stretch are colored pink. Cysteines with extracellular accessibility when the T6SS is active are colored yellow, while the unlabeled ones are colored red Left panel. Docking of the TssM $\mathrm{M}_{26 \mathrm{C}}-\mathrm{Tss} J$ crystal structure in the EM 3D reconstruction of the TssJLM tip complex. Top and bottom panels correspond to side and top views respectively. Right panel. Model of a C10 symmetrized oligomer of $\mathrm{TssM}_{26 \mathrm{Ct}}$ domain. Top and bottom panels correspond to ide and top views respectively.

\section{ONLINE REFERENCES}

1. Durand, E. et al. Structural characterization and oligomerization of the TssL protein, a component shared by bacterial type VI and type IVb secretion systems. J Biol Chem. 287, 14157-68 (2012).

2. Felisberto-Rodrigues, C., et al. Towards a structural comprehension of bacterial type VI secretion systems: characterization of the TssJ-TssM complex of an Escherichia coli pathovar. PLoS pathogens 7, e1002386 (2011).

3. Du, D., et al. Structure of the AcrAB-TolC multidrug efflux pump. Nature. 509, 512-5 (2014).

4. Hodgkinson, J. L., et al. Three-dimensional reconstruction of the Shigella T3SS transmembrane regions reveals 12-fold symmetry and novel features throughout. Nat Struct Mol Biol. 16, 47785 (2009).

5. Low, H. H., et al. Structure of a type IV secretion system. Nature. 508, 550-3 (2014).

6. Shneider, M. M. et al. PAAR-repeat proteins sharpen and diversify the type VI secretion system spike. Nature. 500, 350-3 (2013). 


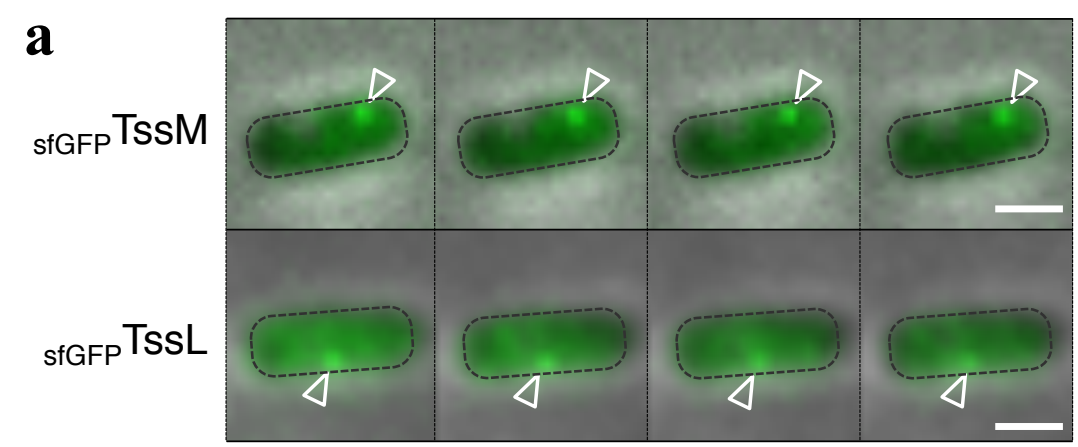

e

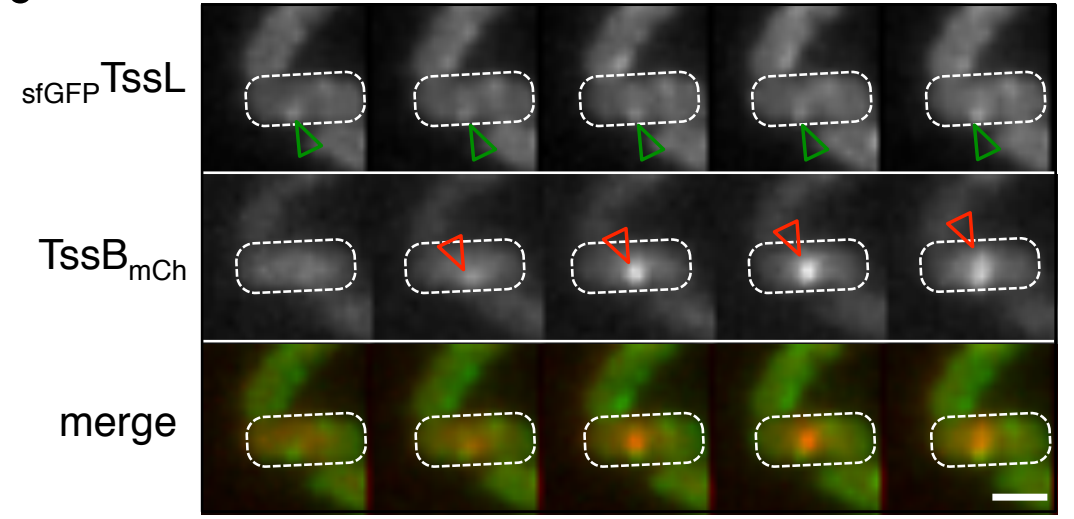

schematic diagram

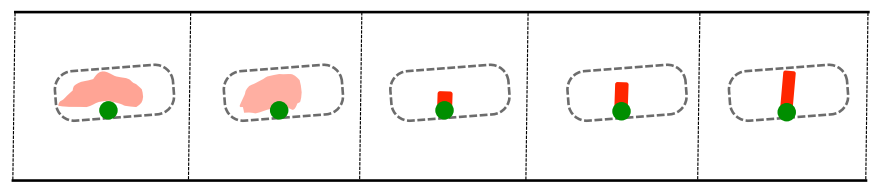

f

sfGFP TSsM sfGFPTSsL

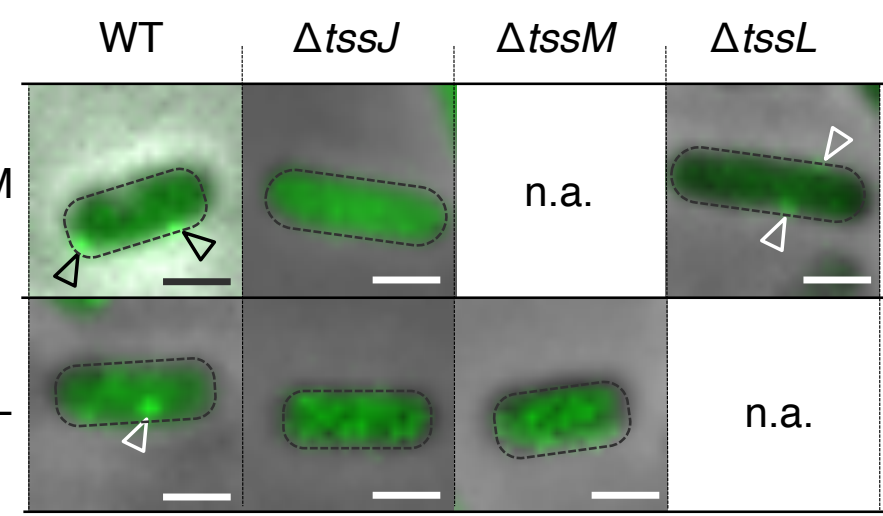

b

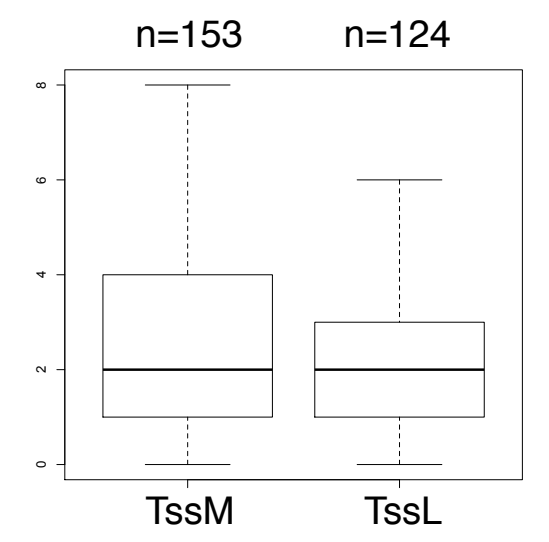

\section{d}

DIC

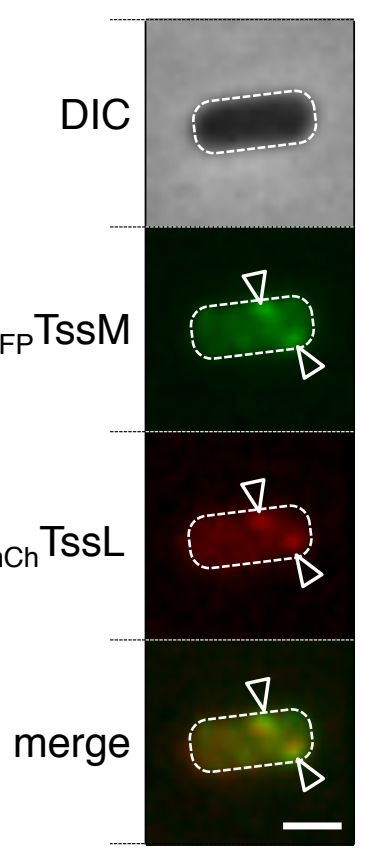

TssM

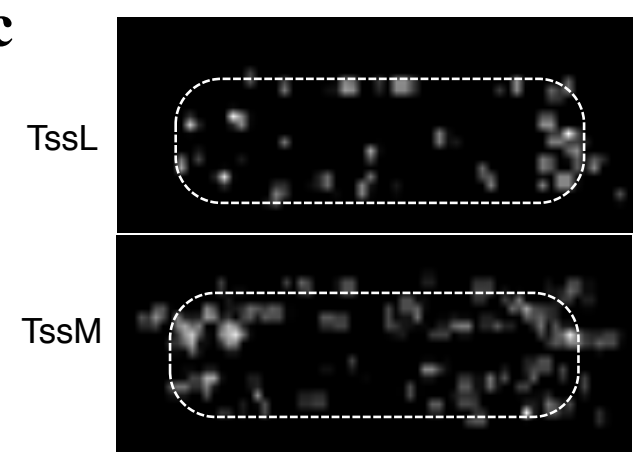

$\mathrm{mCh}^{\text {TssL }}$

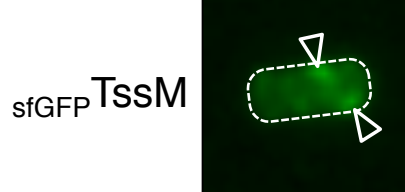

c

g

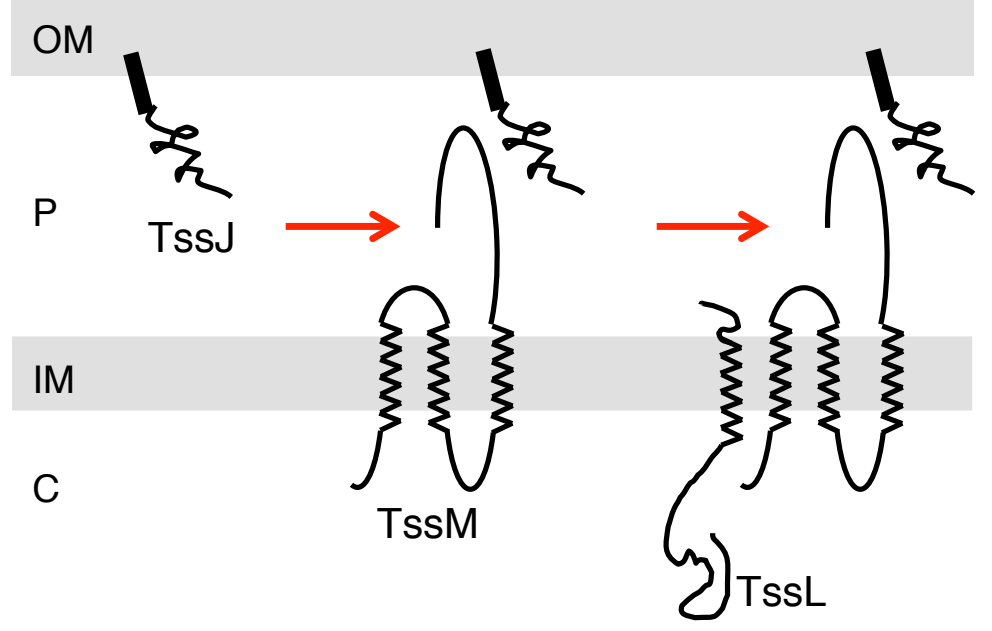




\section{a \\ b}
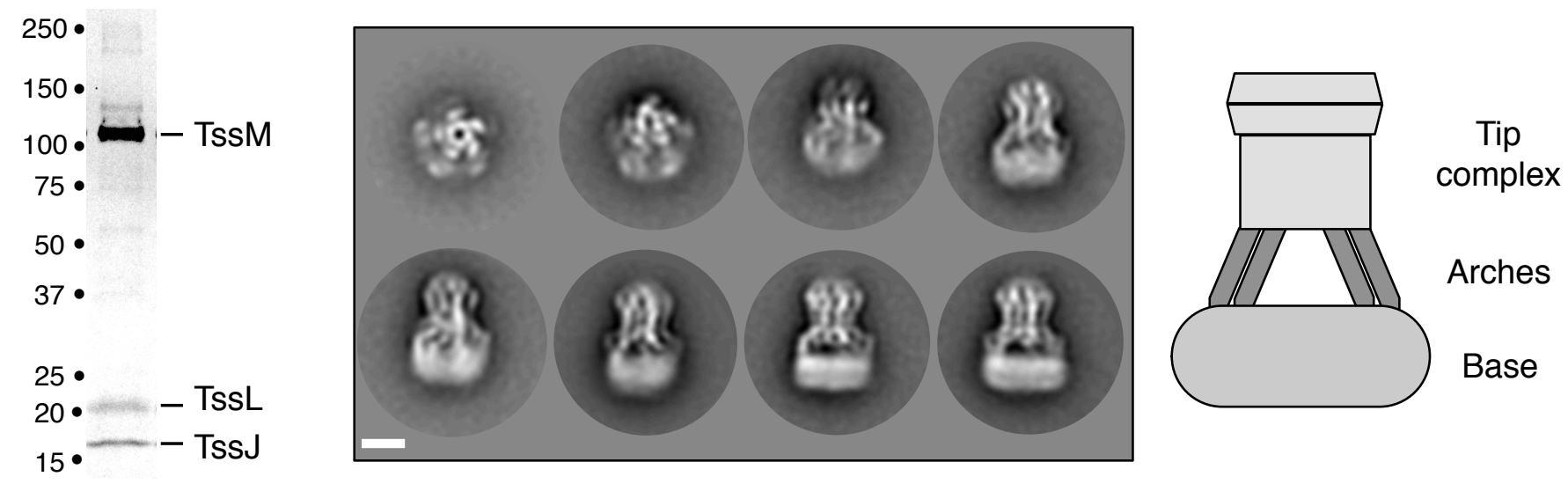

C
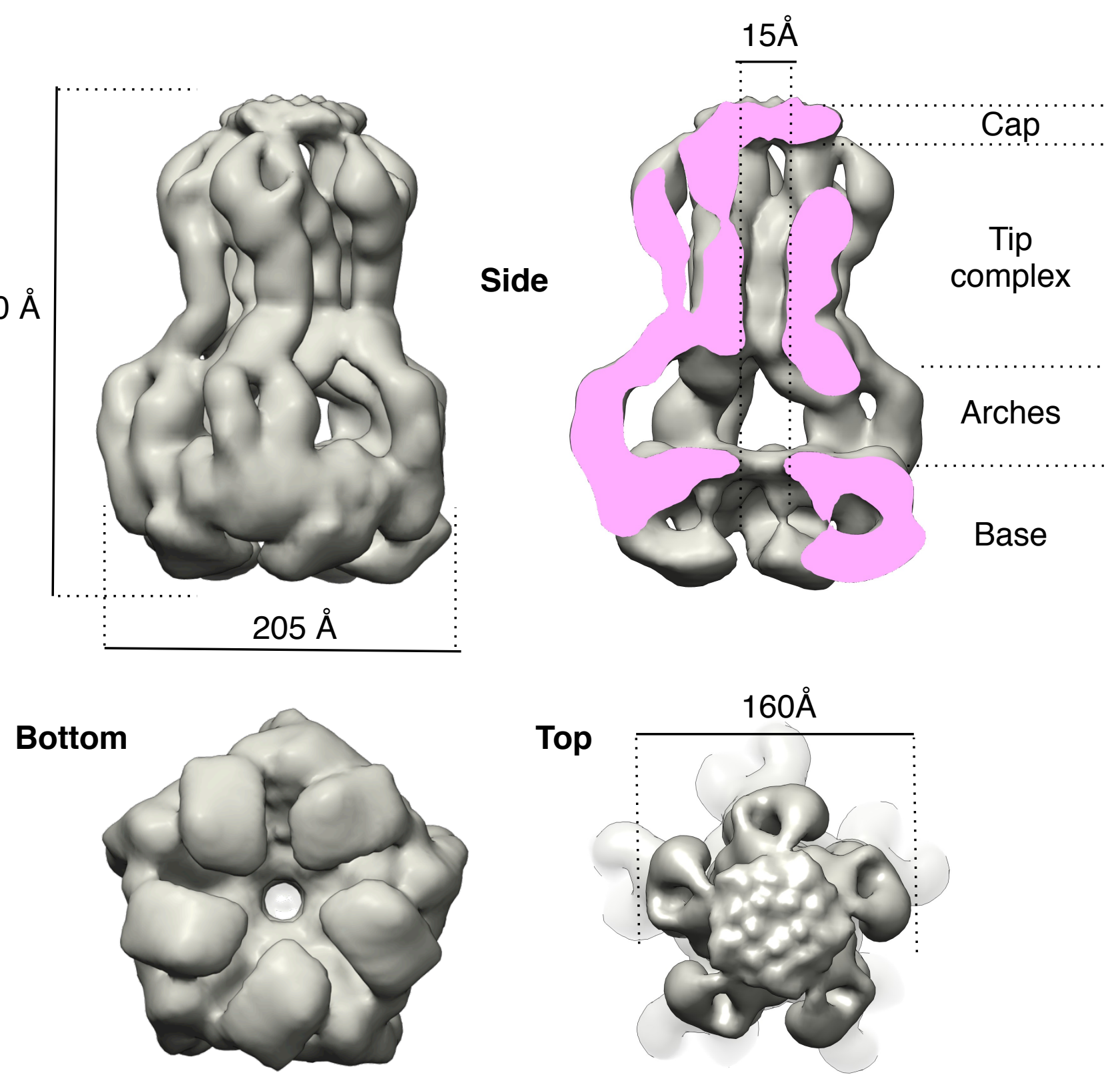
a

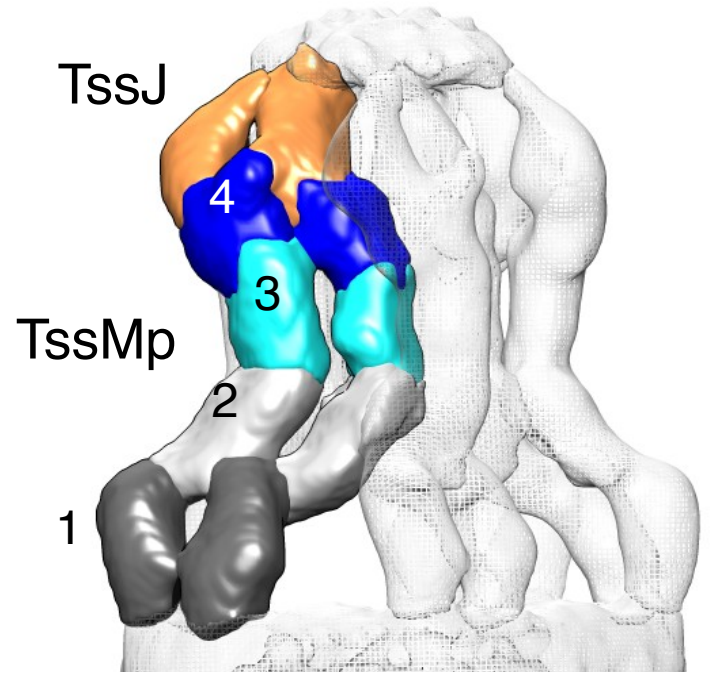

C

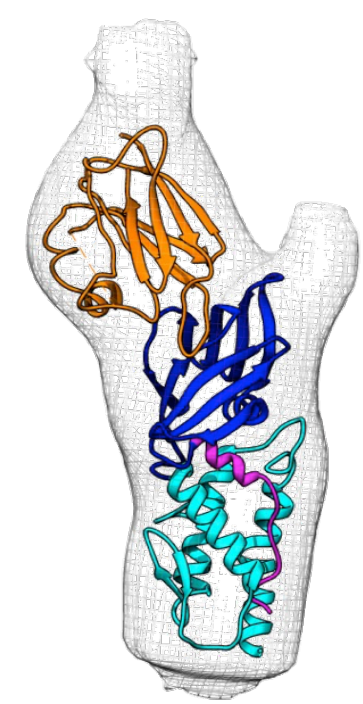

External pillar b

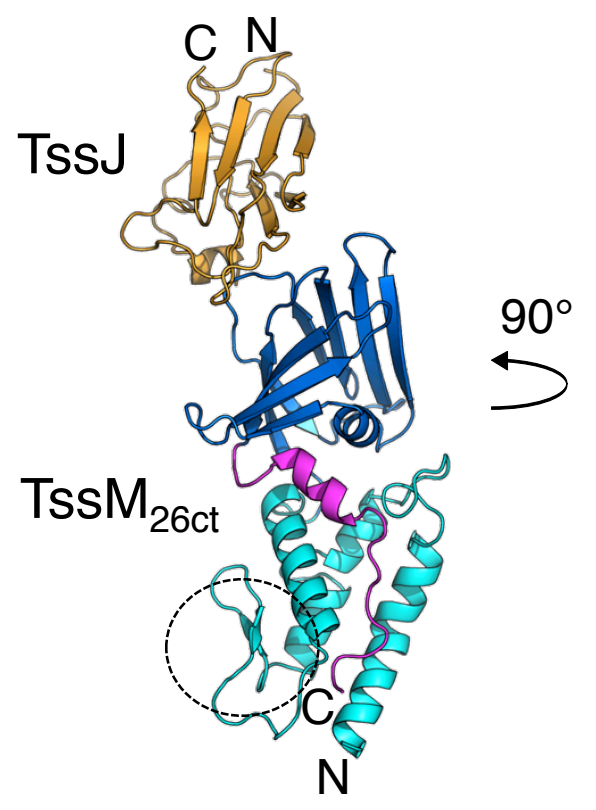

d

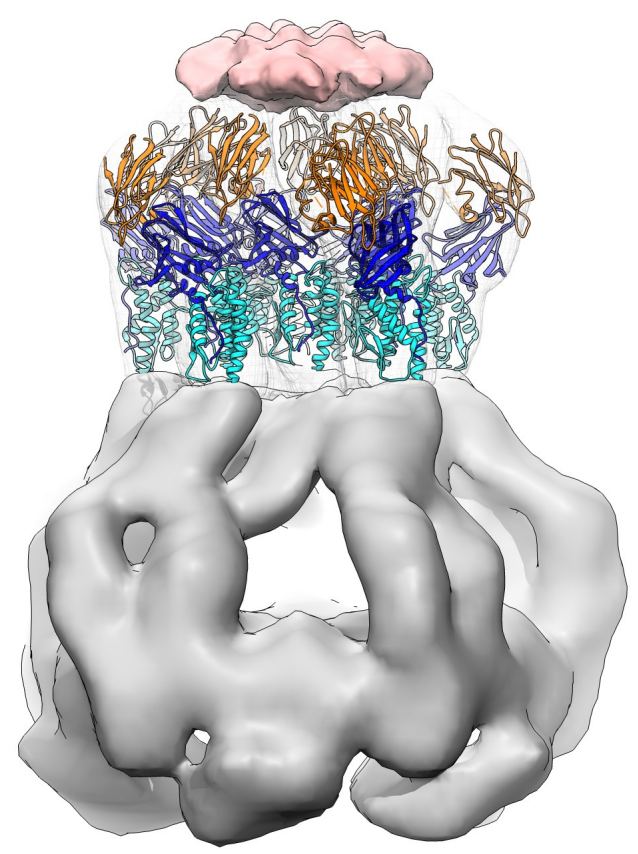

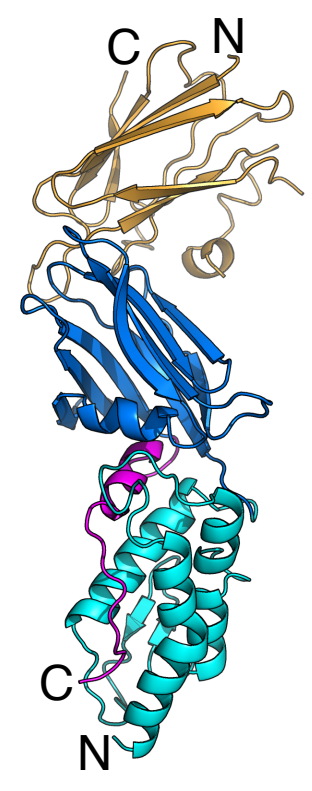
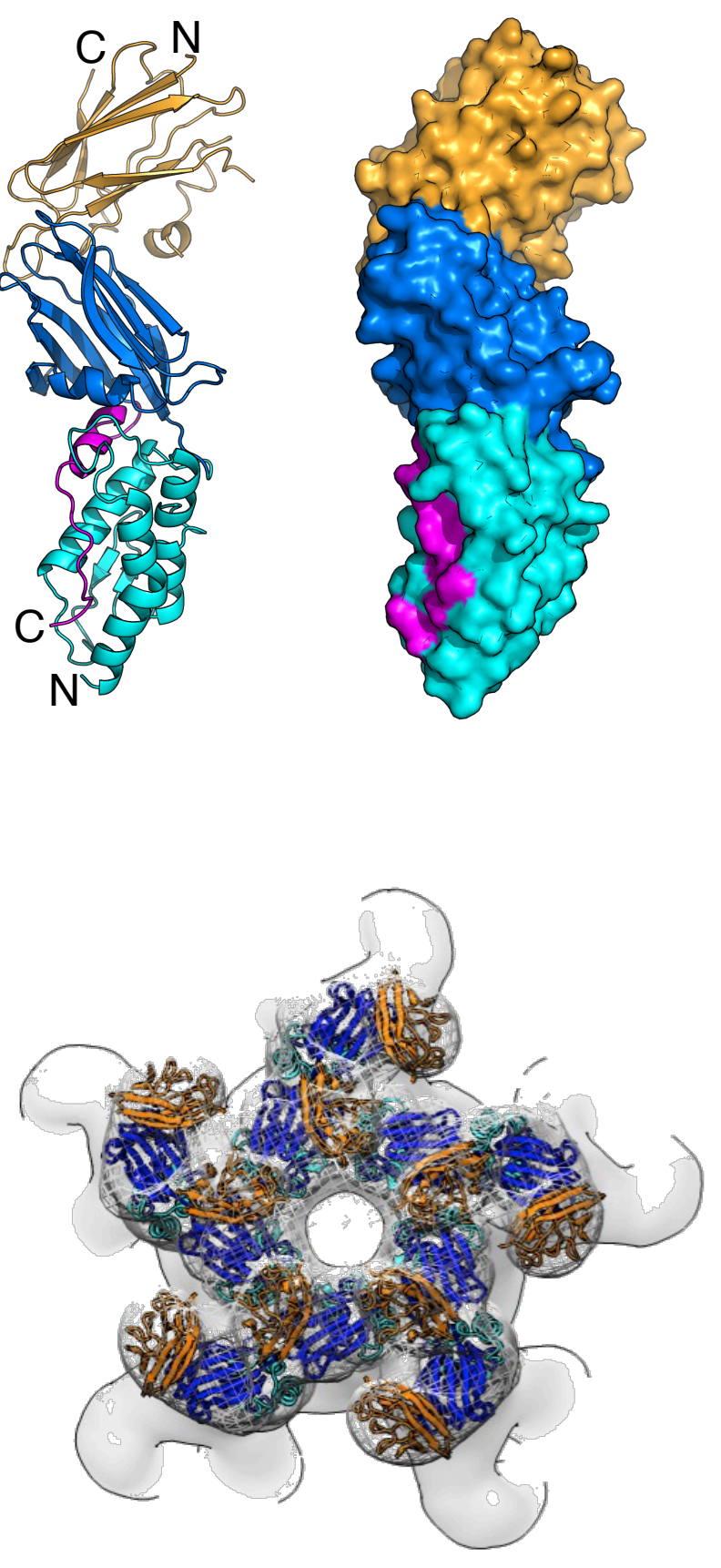


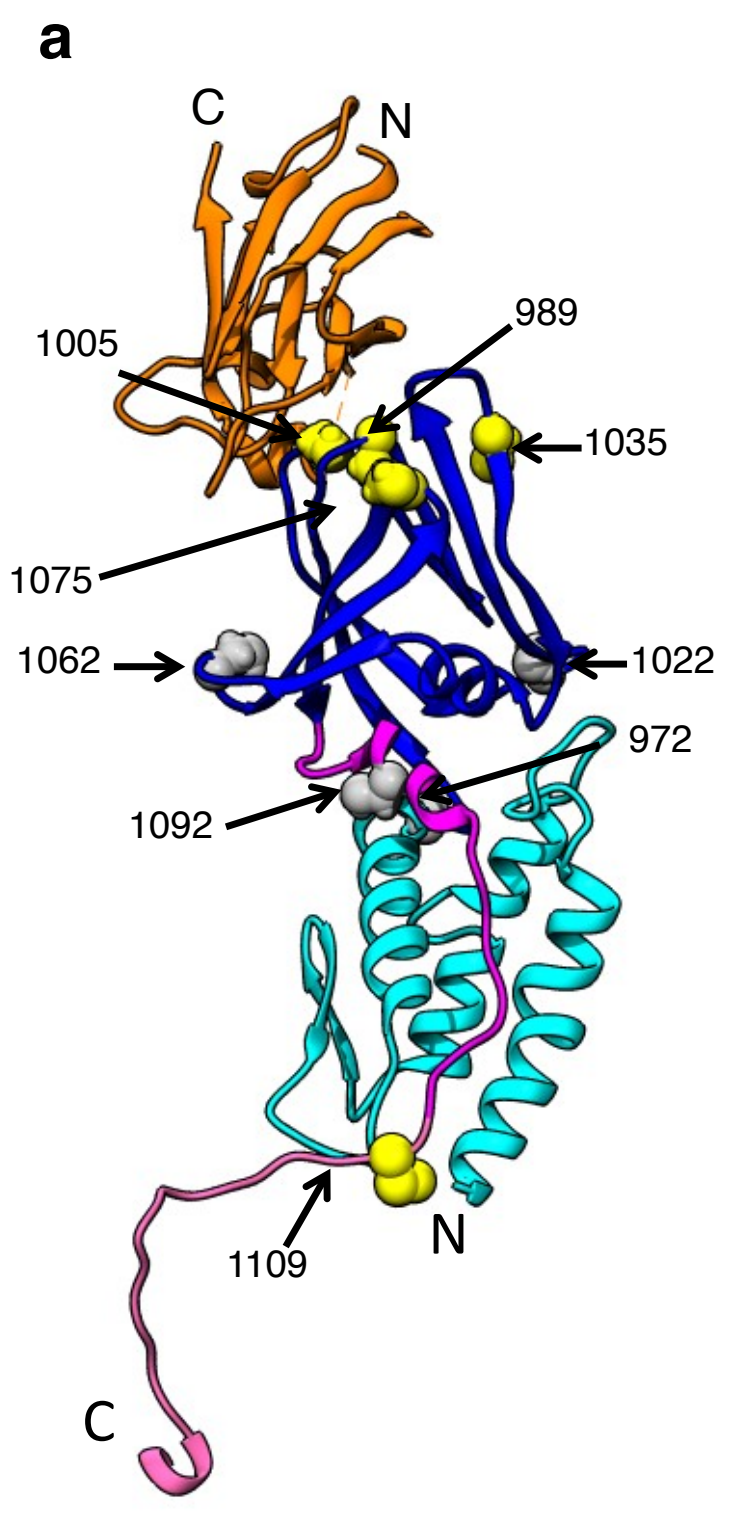

b

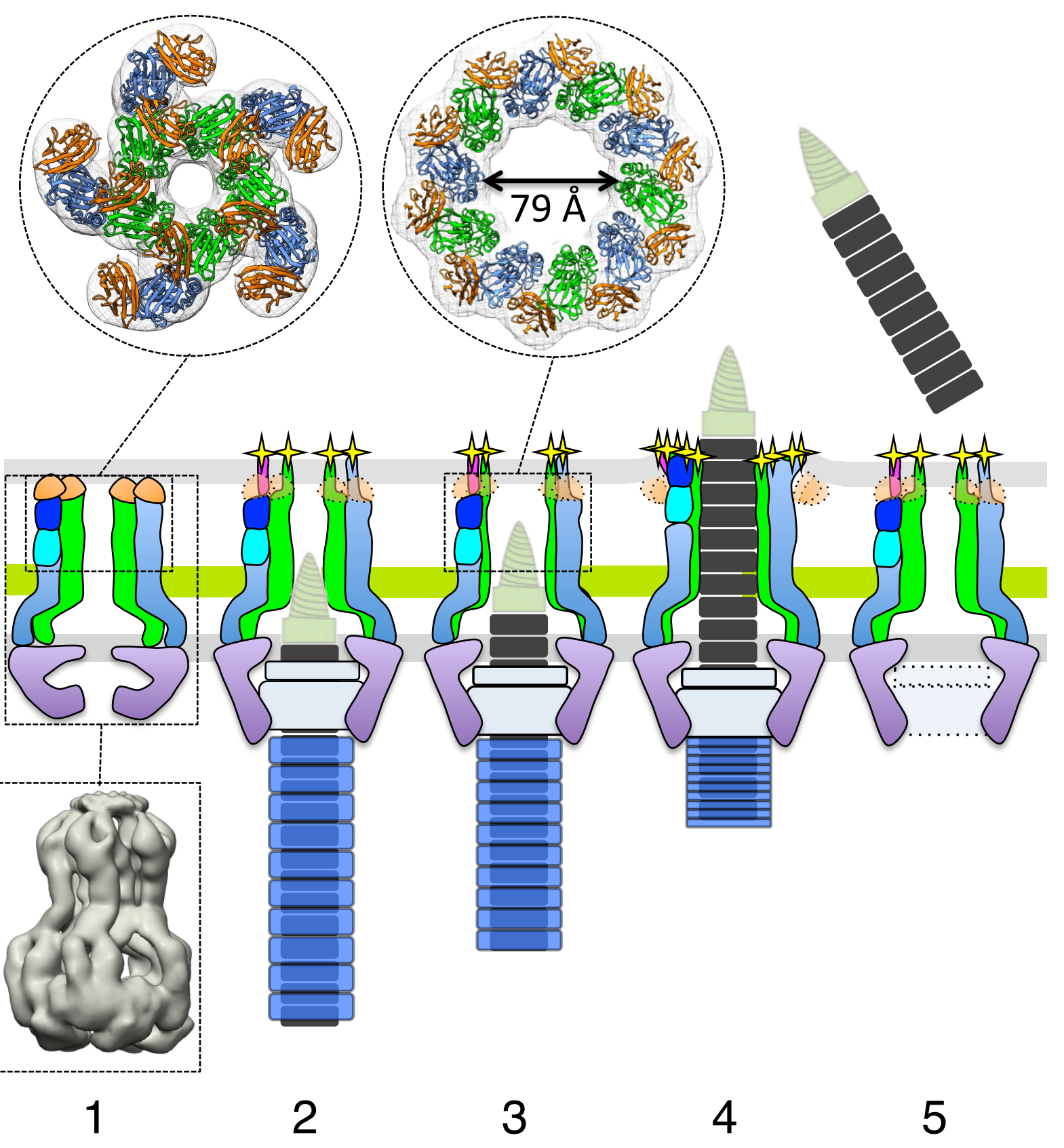

Stage 


\begin{tabular}{|c|c|c|c|}
\hline & $\mathrm{TssM}_{32 \mathrm{Ct}}-\mathrm{nb} 25$ & $\mathrm{TssM}_{26 \mathrm{Ct}}$ & $\mathrm{TssM}_{26 \mathrm{Ct}}{ }^{-T s s J}$ \\
\hline \multicolumn{4}{|l|}{ Data collection $^{\S}$} \\
\hline Space group & $\mathrm{P}_{4}$ & $\mathrm{P} 4_{1} 2_{1} 2$ & $\mathrm{P} 4_{1} 2_{1} 2$ \\
\hline \multicolumn{4}{|l|}{ Cell dimensions } \\
\hline$a, b, c(\AA)$ & $95.2,95.2,172.95$ & $64.0,64.0,249.7$ & $85.5,85.5,256.4$ \\
\hline$\alpha, \beta, \gamma\left({ }^{\circ}\right)$ & $90.0,90.0,120.0$ & $90.0,90.0,90$ & $90.0,90.0,90$ \\
\hline Resolution $(\AA)$ & $50.0-1.92(1.97-1.92)^{*}$ & $30.0-1.51(1.6-1.51)^{*}$ & $50.0-2.24(2.38-2.24)^{*}$ \\
\hline$R_{\text {merge }}$ & $0.079(1.08)$ & $0.067(0.59)$ & $0.067(0.729)$ \\
\hline$I / \sigma I$ & $18.0(2.0)$ & $19.2(3.0)$ & $21.5(3.1)$ \\
\hline Completeness $(\%)$ & $100.0(100.0)$ & $99.9(89.3)$ & $99.7(98.4)$ \\
\hline Redundancy & $11.4(11.3)$ & $9.9(9.9)$ & $10(10)$ \\
\hline \multicolumn{4}{|l|}{ Refinement } \\
\hline Resolution $(\AA)$ & $47.6-1.92(1.97-1.92)^{*}$ & $22.1-1.51(1.55-1.51)^{*}$ & $49.3-2.24(2.3-2.24)^{*}$ \\
\hline No. reflections & $67543(4721)$ & $82127(5359)$ & $46047(3015)$ \\
\hline$R_{\text {work }} R_{\text {free }}$ & $0.184 / 0.21(0.234 / 0.25)$ & $0.192 / 0.202(0.241 / 27.6)$ & $0.208 / 0.228(0.224 / 0.25)$ \\
\hline \multicolumn{4}{|l|}{ No. atoms } \\
\hline Protein & 5522 & 3784 & 5521 \\
\hline Ligand/ion & 15 & 22 & 4 \\
\hline Water & 805 & 536 & 379 \\
\hline \multicolumn{4}{|l|}{ B-factors } \\
\hline Protein & 42.3 & 27.4 & 51.7 \\
\hline Ligand/ion & 98.8 & 55.0 & 81 \\
\hline Water & 49.9 & 39.5 & 63.8 \\
\hline \multicolumn{4}{|l|}{ R.m.s deviations } \\
\hline Bond lengths $(\AA)$ & 0.009 & 0.010 & 0.010 \\
\hline Bond angles $\left({ }^{\circ}\right)$ & 1.05 & 1.03 & 1.19 \\
\hline
\end{tabular}




\begin{tabular}{|c|c|c|c|c|c|c|c|}
\hline $\mathrm{n} 25 / \mathrm{CDR} 3$ & & & $\mathrm{TssM}_{260 \mathrm{C}}$ & & & & \\
\hline number & type & atom & number & type & atom & $\begin{array}{l}\text { distance } \\
(\AA)\end{array}$ & bond \\
\hline 103 & Gly & 0 & 1063 & Ala & $\mathrm{N}$ & 289 & $\mathrm{H}$ \\
\hline 104 & Ile & $\mathrm{CA}$ & 1061 & Gly & o & 3.08 & \\
\hline 105 & Tyr & $\mathrm{N}$ & 1061 & Gly & o & 2.85 & $\mathrm{H}$ \\
\hline 107 & Thr & OG1 & 1060 & Pro & o & 2.67 & $\mathrm{H}$ \\
\hline 107 & Thr & $\mathrm{CG} 2$ & 1061 & Gly & $\mathrm{CA}$ & 3.56 & \\
\hline 109 & Tyr & CE1 & 1067 & Ser & $\mathrm{CB}$ & 3.50 & \\
\hline 109 & Tyr & $\mathrm{OH}$ & 1080 & Tyr & o & 347 & $\mathrm{H}$ \\
\hline 109 & Tyr & $\mathrm{OH}$ & 1081 & Thr & OG1 & 2.64 & $\mathrm{H}$ \\
\hline 110 & Ile & $\mathrm{CD} 1$ & 1062 & Val & CG2 & 3.73 & \\
\hline 113 & Pro & o & 984 & Gly & $\mathrm{CA}$ & 3.12 & \\
\hline 113 & Pro & $\mathrm{O}$ & 985 & Thr & $\mathrm{N}$ & 3.00 & $\mathrm{H}$ \\
\hline 114 & Tyr & CE1 & 982 & Arg & $\mathrm{CD}$ & 3.55 & \\
\hline 114 & Tyr & $\mathrm{CZ}$ & 982 & Arg & $\mathrm{NE}$ & 3.63 & \\
\hline 114 & Tyr & $\mathrm{OH}$ & 1010 & $\operatorname{Trp}$ & NE1 & 2.99 & $\mathrm{H}$ \\
\hline 114 & Tyr & $\mathrm{O}$ & 982 & Arg & $\mathrm{NE}$ & 2.88 & $\mathrm{H}$ \\
\hline 115 & Gly & o & 982 & Arg & $\mathrm{NH} 2$ & 2.76 & $\mathrm{H}$ \\
\hline 116 & Met & $\mathrm{O}$ & 1008 & Pro & $\mathrm{CD}$ & 3.32 & \\
\hline 117 & Asp & OD1 & 982 & Arg & $\mathrm{NH} 2$ & 2.80 & $\mathrm{H}$ \\
\hline
\end{tabular}

\begin{tabular}{|l|c|c|c|c}
\hline $\begin{array}{l}\text { Cysteine } \\
\text { position }\end{array}$ & $\begin{array}{c}\text { Labelled } \\
\text { at rest }\end{array}$ & $\begin{array}{c}\text { Labelled } \\
\text { in action }\end{array}$ & Position & WAS \# \\
\hline 972 & - & - & helix $\alpha 3$ & $107 / 107$ \\
\hline 989 & - & ++ & loop $\beta 3-\beta 4$ & $14 / 10$ \\
\hline 1005 & - & ++ & $\operatorname{loop} \beta 5-\beta 6$ & $76 / 0$ \\
\hline 1019 & - & - & $\operatorname{loop} \beta 6-\beta 7$ & $35 / 35$ \\
\hline 1035 & - & + & $\operatorname{loop} \beta 7-\beta 8$ & $113 / 113$ \\
\hline 1062 & - & - & $\operatorname{loop} \beta 11-\alpha 5$ & $94 / 94$ \\
\hline 1075 & - & ++ & $\operatorname{loop} \beta 10-\beta 11$ & $51 / 51$ \\
\hline 1092 & - & - & helix $\alpha 5$ & $0 / 0$ \\
\hline 1109 & + & ++ & C-terminus & $\mathrm{NA}^{*}$ \\
\hline
\end{tabular}

Tss J

\begin{tabular}{|c|c|c|c|c|c|c|}
\hline TssJ & & & TssM & & & $\begin{array}{c}\text { dist } \\
\text { ance( }(\AA)\end{array}$ \\
\hline 37 & Asn & $\mathrm{Nd} 2$ & 1005 & Asn & $\mathrm{O}$ & $3.06 \mathrm{H}$ \\
\hline 39 & Ser & $\mathrm{Cb}$ & 985 & Thr & $\mathrm{Og} 1$ & 3.80 \\
\hline 43 & Ile & Cg2 & 987 & Ala & $\mathrm{Cb}$ & 3.79 \\
\hline \multirow[t]{2}{*}{45} & Leu & $\mathrm{Cd} 1$ & 985 & Thr & $\mathrm{Cg} 2$ & 3.63 \\
\hline & & $\mathrm{Cb}$ & 1005 & Asn & $\mathrm{Nd} 2$ & 3.56 \\
\hline \multirow[t]{3}{*}{46} & Ser & $\mathrm{Og}$ & 990 & Met & $\mathrm{Ca}$ & 3.14 \\
\hline & & $\mathrm{O}$ & 1005 & Asn & $\mathrm{Nd} 2$ & $3.27 \mathrm{H}$ \\
\hline & & $\mathrm{Og}$ & 1005 & Asn & $\mathrm{Nd} 2$ & $3.19 \mathrm{H}$ \\
\hline 48 & Val & $\mathrm{Cg} 2$ & 1004 & $\mathrm{Val}$ & $\mathrm{Cb}$ & 3.82 \\
\hline \multirow[t]{2}{*}{65} & Tyr & $\mathrm{Cel}$ & 1007 & Met & $\mathrm{Cg}$ & 3.51 \\
\hline & & $\mathrm{Oh}$ & 1007 & Met & $\mathrm{N}$ & $3.30 \mathrm{H}$ \\
\hline 87 & $\operatorname{Trp}$ & Ch2 & 990 & Met & C & 3.56 \\
\hline \multirow[t]{2}{*}{89} & Gln & Oel & 1031 & Thr & $\mathrm{O}$ & $2.87 \mathrm{H}$ \\
\hline & & $\mathrm{Ne} 2$ & 1031 & Thr & $\mathrm{Og} 1$ & $2.79 \mathrm{H}$ \\
\hline 112 & Met & $\mathrm{Ce}$ & 1006 & Gln & $\mathrm{Ne} 2$ & 3.35 \\
\hline 113 & Phe & $\mathrm{O}$ & 1007 & Met & $\mathrm{N}$ & $3.24 \mathrm{H}$ \\
\hline \multirow[t]{6}{*}{114} & Leu & $\mathrm{Cd} 1$ & 1003 & Tyr & $\mathrm{OH}$ & 3.16 \\
\hline & & $\mathrm{N}$ & 1005 & Asn & $\mathrm{O}$ & $3.33 \mathrm{H}$ \\
\hline & & $\mathrm{Cdl}$ & 1006 & Gln & $\mathrm{O}$ & 3.40 \\
\hline & & $\mathrm{O}$ & 1007 & Met & $\mathrm{Cg}$ & 3.51 \\
\hline & & $\mathrm{Cdl}$ & 1008 & Pro & $\mathrm{Cd}$ & 3.73 \\
\hline & Pro & $\mathrm{Cd}$ & 1007 & Met & $\mathrm{Cg}$ & 3.74 \\
\hline
\end{tabular}

Loop L1,2

Loop L3,4

Loop L5,6 
Extended Table 3. Strains, plasmids and oligonucleotides used in this study.

\section{Strains}

Strains

Description and genotype

Source

\section{E. coli $\mathrm{K}-12$}

\section{$\mathrm{DH} 5 \alpha$}

T7 Iq pLysS

BL21(DE3)
F-, $\Delta(\arg F$-lac $) \mathrm{U} 169$, phoA, supE44, $\Delta$ (lacZ)M15, relA, endA, thi, hsdR MiniF lys Y lacl ${ }^{q}\left(\mathrm{Cam}^{\mathrm{R}}\right) /$ fhuA2 lacZ::T7 gene1 [lon] ompT gal sulA11

$R\left(\right.$ mcr-73::miniTn10--Tet $\left.{ }^{\mathrm{S}}\right) 2[\mathrm{dcm}] R\left(\mathrm{zgb}-210: \because\right.$ Tn10--Tet $\left.{ }^{\mathrm{S}}\right)$ endA1 $\Delta(m c r C-m r r) 114 \because \because I S 10$

fhuA2 [lon] ompT gal ( $\lambda$ DE3) [dcm] $\Delta h s d S \lambda$ DE3 = $\lambda$ sBamHIo $\triangle E$ coRI-B int::(lacI::PlacUV5::T7 gene1) i21 $\Delta$ nin5
New England Biolabs

New England Biolabs

New England Biolabs

\section{Enteroaggregative E. coli}

$$
17-2
$$

$17-2 \Delta t s s L$

$17-2 \Delta t s s M$

17-2 gfp-tssL

17-2 gfp-tssM

17-2 tss J-gfp

17-2 gfp-tssL tssB-mCherry
WT enteroaggregative Escherichia coli

17-2 deleted of the $t s s L$ gene of the scil T6SS gene cluster 17-2 deleted of the $t s s M$ gene of the sci1 T6SS gene cluster gfp-mut 2 inserted downstream the start codon of tss $L$ in 17-2 $g f$-mut 2 inserted downstream the start codon of tss $M$ in 17-2 $g f p-m u t 2$ inserted upstream the stop codon of $t s S J$ in 17-2 $m$ Cherry inserted upstream the stop codon of $t s s B$ in 17-2 gfp-tssL
Arlette Darfeuille-Michaud Aschtgen et al., 2010

Aschtgen et al., 2010 This study This study This study This study 
17-2 gfp-tssM mCherry-tssL 17-2 $\Delta t s s M g f p$-tssL

17-2 $\Delta t s s J g f p-t s s L$

17-2 $\Delta t s s L$ gfp-tssM

17-2 $\Delta t s s J g f p-t s s M$ $m$ Cherry inserted downstream the start codon of tss $L$ in 17-2 gfp-tss $M$ gfp-mut 2 inserted downstream the start codon of tss $L$ in 17-2 $\Delta t s s M$ gfp-mut 2 inserted downstream the start codon of tss $L$ in 17-2 $\Delta t s s J$ $g f p$-mut 2 inserted downstream the start codon of $t s s M$ in 17-2 $\Delta t s s L$ gfp-mut 2 inserted downstream the start codon of tss $M$ in 17-2 $\Delta t s s J$
This study This study This study This study This study

\section{Plasmids}

\section{Vectors}

\section{Description}

Source

\section{Expression vectors}

pUC-Hcp ${ }_{\mathrm{FL}}$ pIBA-TssM scil hcp gene cloned into pUC12, Plac, C-terminal FLAG epitope scil tssM gene cloned into pASK-IBA37(+), Ptet, N-terminal FLAG epitope
Aschtgen et al., 2008 This study

Vectors for TssJLM and protein purification

\begin{tabular}{|c|c|c|}
\hline pRSF-Duet1 & Expression vector, lacI, $\mathrm{P} T 7, \mathrm{Kan}^{\mathrm{R}}$ & Addgene \\
\hline pRSF-Tss J ${ }^{\mathrm{S}}{ }^{\mathrm{F}} \mathrm{L}-{ }^{\mathrm{H}} \mathrm{M}$ & tssJ-StrepII, FLAG-tssLL and 6×His-tss $M$ cloned into pRSF-Duet1 & This study \\
\hline pETG20A & Gateway destination vector, TRX $-6 \times$ His followed by a TEV cleavage site & Arie Gerlof \\
\hline pHEN6-nb25 & pHEN6 vector encoding the nb25 nanobody & NGuyen et al., 2015 \\
\hline pETG20A-TssJ & $\begin{array}{l}\text { scil tss } J \text { mature form cloned into } \mathrm{pETG} 20 \mathrm{~A} \\
\text { N-terminal TRX-His-TEV epitope }\end{array}$ & Felisberto-Rodrigues et al., 2011 \\
\hline pETG20A-TssMp & $\begin{array}{l}\text { scil tss } M \text { periplasmic domain (aa 386-1129) cloned into pETG20A, } \\
\text { N-terminal TRX-His-TEV epitope }\end{array}$ & Felisberto-Rodrigues et al., 2011 \\
\hline
\end{tabular}




\section{Vectors for chromosomal insertions}

\begin{tabular}{|c|c|c|}
\hline pKD4 & $\begin{array}{l}\operatorname{Kan}^{\mathrm{R}} \text { cassette flanked by FRT recombination sites, } \\
\text { used for chromosomal deletion }\end{array}$ & Datsenko and Wanner, 2000 \\
\hline pKD4 $4_{\text {Nter-gfp }}$ & $\begin{array}{l}g f p-m u t 2(s f-g f p) \text { gene cloned upstream the } \mathrm{Kan}^{\mathrm{R}} \text { cassette in pKD4, } \\
\text { used for chromosomal insertion of } g f p-m u t 2(\mathrm{~N} \text {-terminal GFP) }\end{array}$ & This study \\
\hline pKD4 $4_{\text {Cter-mCherry }}$ & $\begin{array}{l}m \text { Cherry gene cloned downstream the } \operatorname{Kan}^{\mathrm{R}} \text { cassette in pKD4, } \\
\text { used for chromosomal insertion of } m \text { Cherry (C-terminal mCherry) }\end{array}$ & This study \\
\hline pCP20 & $\mathrm{Amp}^{\mathrm{R}}, \mathrm{Cm}^{\mathrm{R}}, \mathrm{FRT}$ recombinase gene & Datsenko and Wanner, 2000 \\
\hline
\end{tabular}

\section{Oligonucleotides}

\section{Name}

Destination

Sequence $\left(5^{\prime} \rightarrow 3^{\prime}\right)$

\section{For strain construction ${ }^{\text {a }}$}

\begin{tabular}{|c|c|c|}
\hline 5-tssB-mCherry & insertion of $m$ Cherry at the $3^{\prime}$ end of $t s s B$ & $\begin{array}{l}\text { CCGGCACTGAGTCAGACGCTGCGTGATGAACTGCGTGCACTGGTG } \\
\text { CCGGAAAAGGCGGCAGCGGCCGGCGGAGGG }\end{array}$ \\
\hline 3-tssB-mCherry & insertion of $m$ Cherry at the $3^{\prime}$ end of $t s s B$ & $\begin{array}{l}\text { ГTCTTTTCTTTCTGTACAGACATCAGCATTTTCTCTCGTAA } \\
\text { AACATATGAATATCCTCCTTAGTTCCTATTCCGAAGTTCC }\end{array}$ \\
\hline$-g f p-t s s L$ & insertion of $g f p$ & $\begin{array}{l}\text { CTACACCCCGGCATCGCTGGGAGATGTGAAACTGGAACTTTTTGC } \\
\text { GGGACATGACGATTGTGTAGGCTGGAGCTGCTTCGAAGTTCCTATAC }\end{array}$ \\
\hline
\end{tabular}




\begin{tabular}{|c|c|c|}
\hline 5-gfp-tss $M$ & insertion of $g f p$-mut 2 at the $5^{\prime}$ of $t s s M$ & TTCTCATCCGGAGAAGAACATTTTATCAGTACTGTTACATCAGGAA \\
\hline & $\underline{\mathrm{ACC}}$ & AATGAATAACGATTGTGTAGGCTGGAGCTGCTTCGAAGTTCCTATAC \\
\hline 3-gfp-tss $M$ & insertion of $g f p-m u t 2$ at the 5 ' of $t s s M$ & CACACCAATAAATACAATCCCCGGTCGCCCAAAGCGACCAGACAG \\
\hline & & ACAGGCCAGTTTATTCCCTCCGCCGGCCGCTGC \\
\hline $5-t s s J-g f p$ & insertion of $g f p-m u t 2$ at the 3 ' of $t s s J$ & $\begin{array}{l}\text { CACGCCCCGTCTTATTGAAGTATCCGGTAACACCCTGACCCTGTTA } \\
\text { CCGGTGAAGGATAAAGCAGCGGCCGGCGGAGGG }\end{array}$ \\
\hline 3-tssJ-gfp & $\begin{array}{r}\text { insertion of } g f p-m u t 2 \text { at the } 3 \text { ' of } t s S J \\
\text { AGGGAAC }\end{array}$ & $\begin{array}{l}\text { GTTTAACTCCAGCCCGCCAGTGAAATTGCCATAGAGAATTTCATAA } \\
\text { GCGGCATCACATATGAATATCCTCCTTAGTTCCTATTCCGAAGTTCC }\end{array}$ \\
\hline
\end{tabular}

For plasmid construction $^{\text {b,c,d }}$

\begin{tabular}{|c|c|c|}
\hline \multirow[t]{3}{*}{ IBA37-TssM-F } & \multicolumn{2}{|c|}{ insertion of $t s s M$ with $\mathrm{N}$-terminal FLAG epitope into pASK-IBA37 } \\
\hline & \multirow{2}{*}{\multicolumn{2}{|c|}{ 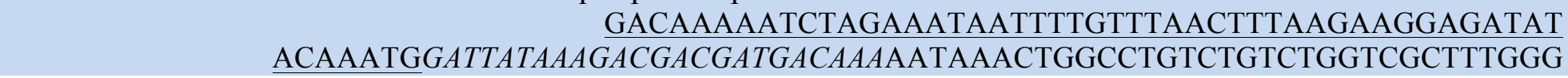 }} \\
\hline & & \\
\hline & \multicolumn{2}{|c|}{ insertion of $t s s M$ with N-terminal FLAG epitope into pASK-IBA37 } \\
\hline & \multicolumn{2}{|c|}{$\begin{array}{l}\text { GATGGTGATGGTGAT } \\
\text { CACGGTATCCCCGG }\end{array}$} \\
\hline D4-Nt-sfGFP-5 & insertion of $g f p$-mut 2 into $\mathrm{pKD} 4$ & \multirow{2}{*}{$\begin{array}{l}\text { CGGAATAGGAACTAAGGAGGATATTCATATGTCTAAAGGTGAAGAAC } \\
\text { TGTTCACCG }\end{array}$} \\
\hline & & \\
\hline KD4-Nt-sfGFP-3 & insertion of $g f p$-mut 2 into $\mathrm{pKD} 4$ & $\frac{\text { CTGACATGGGAATTAGCCATGGTCCCCTCCGCCGGCCGCTGCTTTGTAGA }}{\text { GCTCATCCATGCCG }}$ \\
\hline KD4-Ct-mCherry & insertion of $m$ Cherry into pKD4 & GCAGCATTACACGTCTTGAGCGATTGCAGCGGCCGGCGGAGG \\
\hline KD4-Ct- mCherry-3 & insertion of $m$ Cherry into $\mathrm{pKD} 4$ & $\begin{array}{l}\frac{\text { CTTCGAAGCAGCTCCAGCCTACACTTACTTGTACAGCTCGTCCATGCC }}{\text { GCC }} \\
\text {. }\end{array}$ \\
\hline 5-pETG20-TssM $26 \mathrm{Ct}$ & insertion of $t s S M$ (aa 868-1107) into pETG20A & $\begin{array}{l}\text { GACAAGTTTGTACAAAAAAGCAGGCTTAGAAAACCTGTACT } \\
\text { TCCAGGGTACGCCCGCGGCAGAAAGTCTG }\end{array}$ \\
\hline 3-pETG20-TssM $26 \mathrm{Ct}$ & insertion of $t s s M$ (aa 868-1107) into pETG20A & $\begin{array}{l}\text { CCACTTTGTACAAGAAAGCTGGGTTTATTCGAAAACCGTTT } \\
\text { CCGGCAGAAC }\end{array}$ \\
\hline RSF-Js-Fwd & \multicolumn{2}{|r|}{ ATGGTACATATGATGGCGATTATCGCTGGTAAGGCTGGTGG } \\
\hline
\end{tabular}




\begin{tabular}{|c|c|c|}
\hline & & AATTCCATATGGCGATTATCGCTGGTAAGGCTGGTTACGGTC \\
\hline RSF-Js-Rev & insertion of StrepII-tagged $t s S J$ into pRSF-Duet & $\begin{array}{l}\text { AATCCGCTCGAGTCATTTTTCGAACTGCGGGTGGCTCCATTT } \\
\text { ATCCTTCACCGGTAACAGGGTCAGGGTG }\end{array}$ \\
\hline RSF-flL-Fwd & insertion of FLAG-tagged tss $L$ into pRSF-Duet & $\begin{array}{l}\text { GCAGTTCGAAAAATGACTCGAGAAGGAGATATACCATGGA } \\
\text { TTATAAAGATGACGATGACAAGAATAAACCTGTTATCTCCCGGGC }\end{array}$ \\
\hline RSF-flL-Rev & insertion of FLAG-tagged $t s s L$ into pRSF-Duet & $\frac{\text { ATGTATATCTCCTTTTATCCCTGCCCGGTAAGCCGTGCCACC }}{\text { TGGTCTG }}$ \\
\hline RSF-hM-Fwd & insertion of $6 \times$ His-tagged tss $M$ into pRSF-Duet & $\begin{array}{l}\text { GGATAAAAGGAGATATACATATGCATCACCATCATCACCACC } \\
\text { ATCACAATAAACTGGCCTGTCTGTCTGGTC }\end{array}$ \\
\hline RSF-hM-Rev & insertion of $6 \times$ His-tagged tss $M$ into pRSF-Duet & $\begin{array}{l}\text { GCGGTTTCTTTACCAGACTCGAGTCAGTCAGTCTCCTCCACG } \\
\text { GTATCC }\end{array}$ \\
\hline
\end{tabular}

For site-directed mutagenesis $^{\mathrm{e}}$

$\begin{array}{lll}\text { A-TssJ C1S } & \text { Cys1-to-Ser substitution in } t s s J & \text { TTATCAGGATCCGGTCTGACGCAAAGAGTGGCAGACGGTACGGTATCTGC } \\ \text { B-TssJ C1S } & \text { Cys1-to-Ser substitution in } t s s & \text { GTCAGACCGGATCCTGATAACGACAGGGAAAACAACGCAATAAT } \\ \text { A-TssM-C727S } & \text { Cys727-to-Ser substitution in } t s s M & \text { GAATACGCTGGCGGTTCAGGGATCCACTGGCCAGCCCCGGGAAG } \\ \text { B-TssM-C727S } & \text { Cys727-to-Ser substitution in } t s s M & \text { CTTCCCGGGGCTGGCCAGTGGATCCCTGAACCGCCAGCGTATTC } \\ \text { A-TssM-T972C } & \text { Thr972-to-Cys substitution in } t s s M & \text { ATAGCGGATGTGGCGTTCACCTGTGGTAACGCGGGGCTGCATTTTG } \\ \text { B-TssM-T972C } & \text { Thr972-to-Cys substitution in } t s s M & \text { CAAAATGCAGCCCCGCGTTACCACAGGTGAACGCCACATCCGCTAT } \\ \text { A-TssM-V989C } & \text { Val989-to-Cys substitution in } t s s M & \text { CGCCCGGGAACTGCTGCCGGTTGTATGCAGACGACGCTGATAAC } \\ \text { B-TssM-V989C } & \text { Val989-to-Cys substitution in } t s s M & \text { GTTATCAGCGTCGTCTGCATACAACCGGCAGCAGTTCCGGGCG } \\ \text { A-TssM-N1005C } & \text { Asn1005-to-Cys substitution in } t s s M & \text { GATAATCAGAAACTGATTTATGTTTGTCAGATGCCGGTATGGAAGCG } \\ \text { B-TssM-N1005C } & \text { Asn1005-to-Cys substitution in } t s s M & \text { CGCTTCCATACCGGCATCTGACAAACATAAATCAGTTTCTGATTATC } \\ \text { A-TssM-T1019C } & \text { Thr1019-to-Cys substitution in } t s s M & \text { GATTTACCTGGCCGGCTGATTGTGAAGCACCTGGCGCCAGTTTAAG } \\ \text { B- TssM-T1019C } & \text { Thr1019-to-Cys substitution in } t s s M & \text { CTTAAACTGGCGCCAGGTGCTTCACAATCAGCCGGCCAGGTAAATC } \\ \text { A-TssM-T1035C } & \text { Thr1035-to-Cys substitution in } t s s M & \text { GGGTAAGCACTCAGGCCGTGTCCGTCAGTATGCAGACCTGCCGGG } \\ \text { B- TssM-T1035C } & \text { Thr1035-to-Cys substitution in } t s s M & \text { CCCGGCAGGTCTGCATACTGACGGGACACGGCCTGAGTGCTTTACCC } \\ \text { A-TssM-V1062C } & \text { Val1062-to-Cys substitution in } t s s M & \text { GACGGAAAGCCGCACCGGTGTTTGCAGTGGCTGGAGCCTGAG } \\ \text { B- TssM-V1062C } & \text { Val1062-to-Cys substitution in } t s s M & \text { CTCAGGCTCCAGCCACTGGCAAACACCGGTGCGGCTTCCGTC } \\ \text { A-TssM-G1075C } & \text { Gly1075-to-Cys substitution in } t s s M & \text { CTGAGCTGGCAGGCGCAGGACTGTCGTATGCTGAATTACACACTGC } \\ \text { B- TssM-G1075C } & \text { Gly1075-to-Cys substitution in } t s s M & \text { GCAGTGTGTAATTCAGCATACGACAGTCCTGCGCCTGCCAGCTCAG }\end{array}$


A-TssM-V1092C Val1092-to-Cys substitution in tssM B- TssM-V1092C Val1092-to-Cys substitution in tssM A-TssM-S1109C Ser1109-to-Cys substitution in tssM B-TssM-S1109C
GGGGAAGGGCCGCTTTGTTTGCTGAAACTCCGCAATTTTG

CAAAATTGCGGAGTTTCAGCAAACAAAGCGGCCCTTCCCC

GAAACGGTTTTCGAACTCTGCGGCACGTCAGCGTTTAC

GTAAACGCTGACGTGCCGCAGAGTTCGAAAACCGTTTC

\footnotetext{
${ }^{a}$ Sequences corresponding to the downstream and upstream regions of the gene to be deleted underlined.

${ }^{\mathrm{b}}$ Sequence annealing on the target plasmid underlined.

${ }^{\mathrm{c}}$ Restriction sites in Bold.

${ }^{\mathrm{d}}$ FLAG, StrepII or $6 \times$ His tag coding sequence italicized.

${ }^{\mathrm{e}}$ mutagenized codon underlined.
} 
a
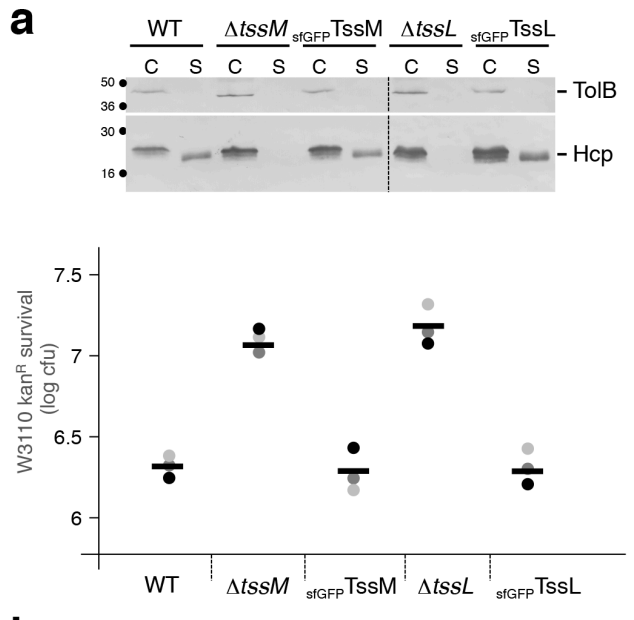

b

$$
\begin{aligned}
& \text { 50. } \frac{\text { WT }}{\mathrm{C} S \mathrm{~S}} \frac{\Delta t s S J}{\mathrm{C} S \mathrm{~S}} \frac{\mathrm{TsSJ}_{\mathrm{SfGFP}}}{\mathrm{C} S \mathrm{~S}} \frac{\mathrm{TsS}_{\mathrm{mCh}}}{\mathrm{C} S \mathrm{~S}} \\
& { }_{30}^{36} \\
& { }_{16}^{30} \bullet
\end{aligned}
$$

C

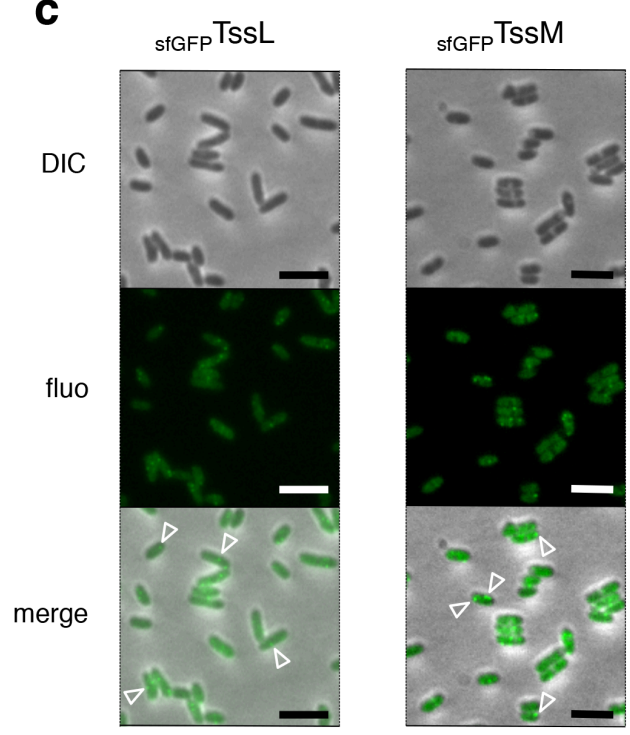

d

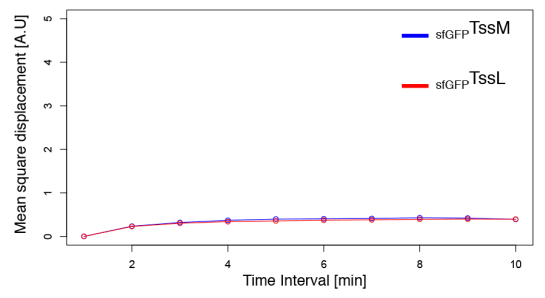

e

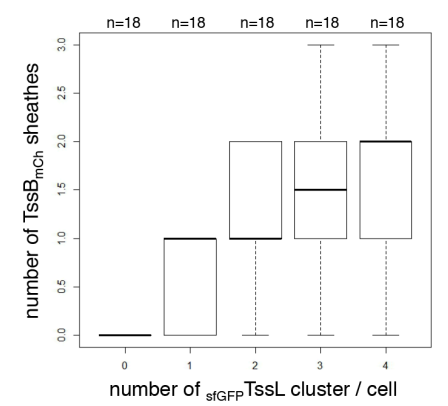

$\mathbf{f}$

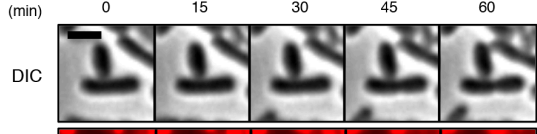

TssB $_{\text {man }}$ (-)

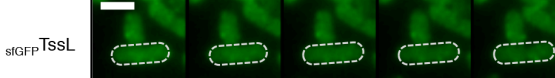

merge 0,0 ,

\begin{tabular}{|c|c|c|c|c|}
\hline schematic \\
diagram
\end{tabular}

g

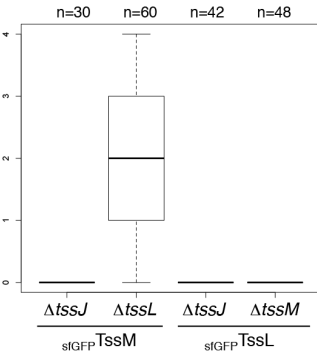

Extended Data Figure 1 
a

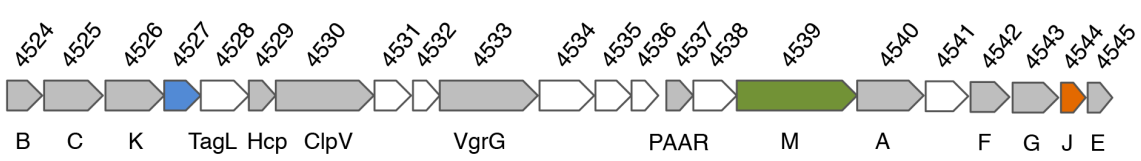

b

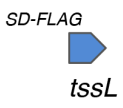

C

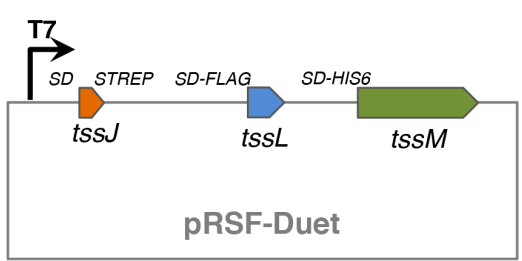

SD-HISG

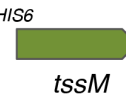

d

TssJ

SPCYS STREP TSSL N N FIAG
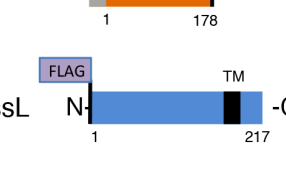

TssM N
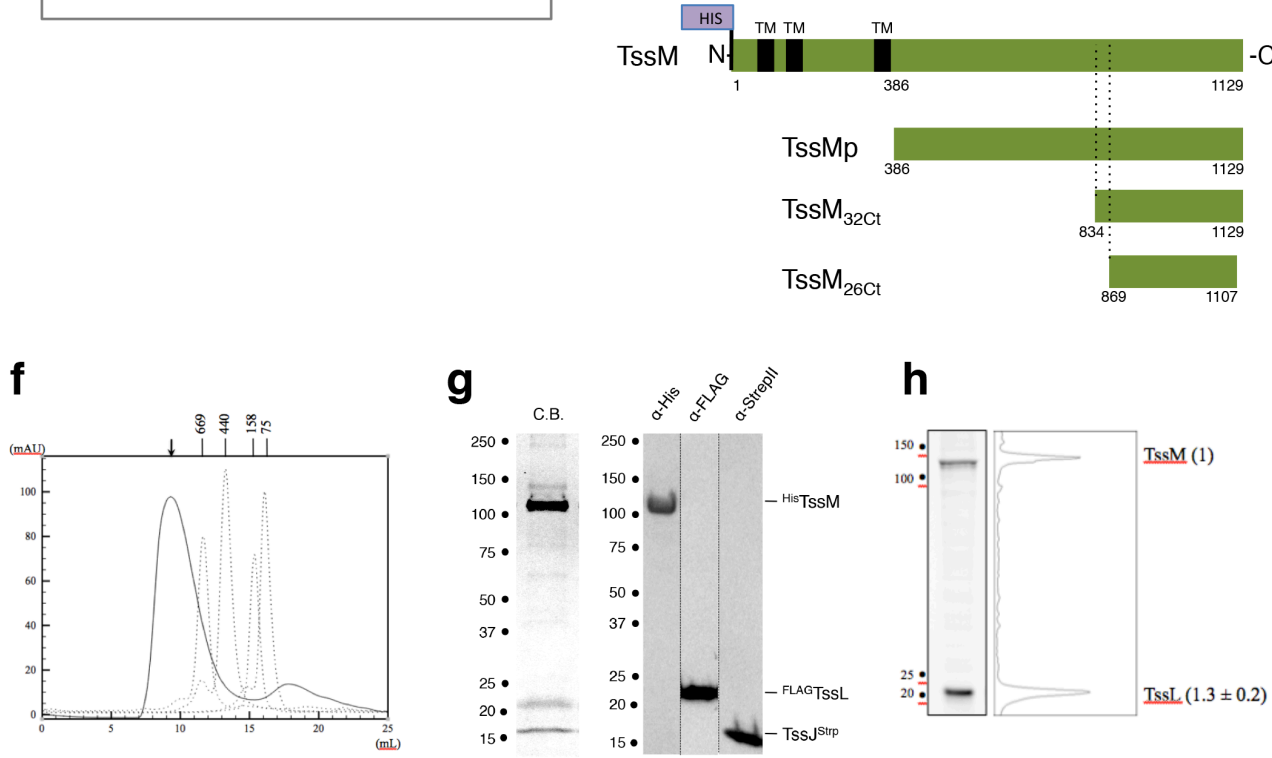

\section{g}

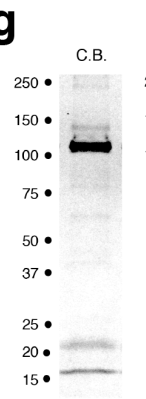

h

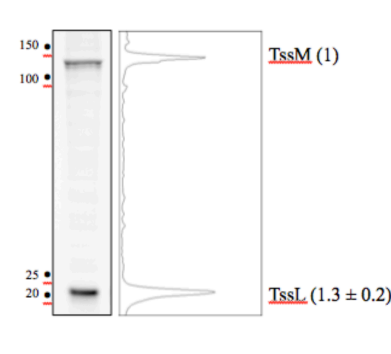

Extended Data Figure 2 
a

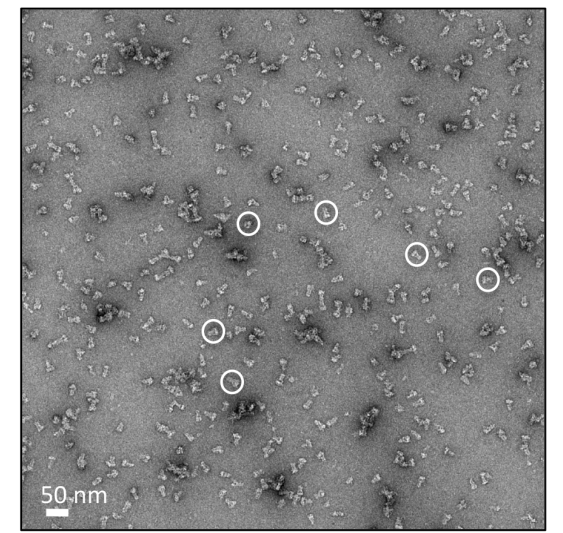

C

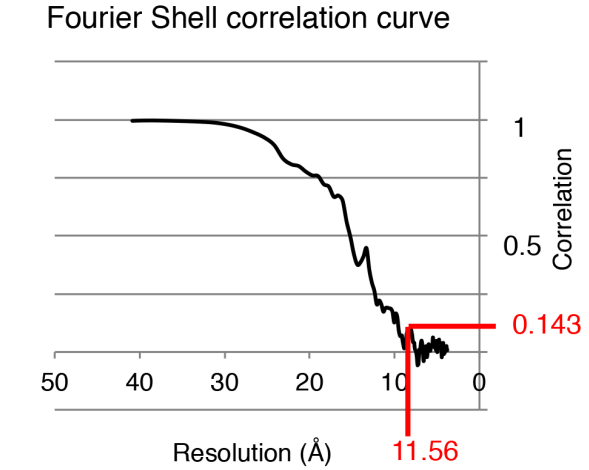

e

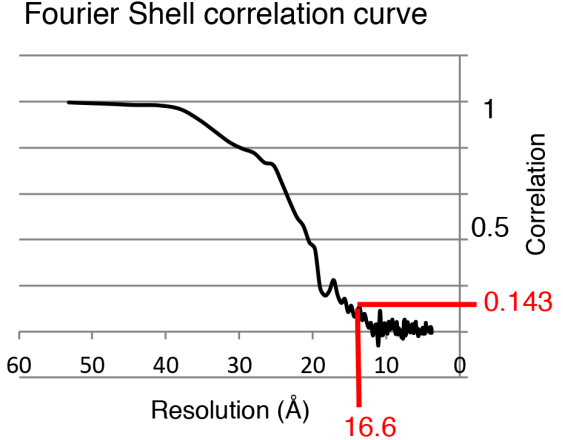

b Rotational autocorrelation function

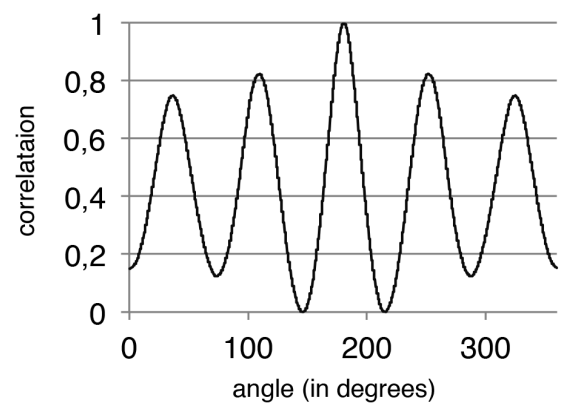

d
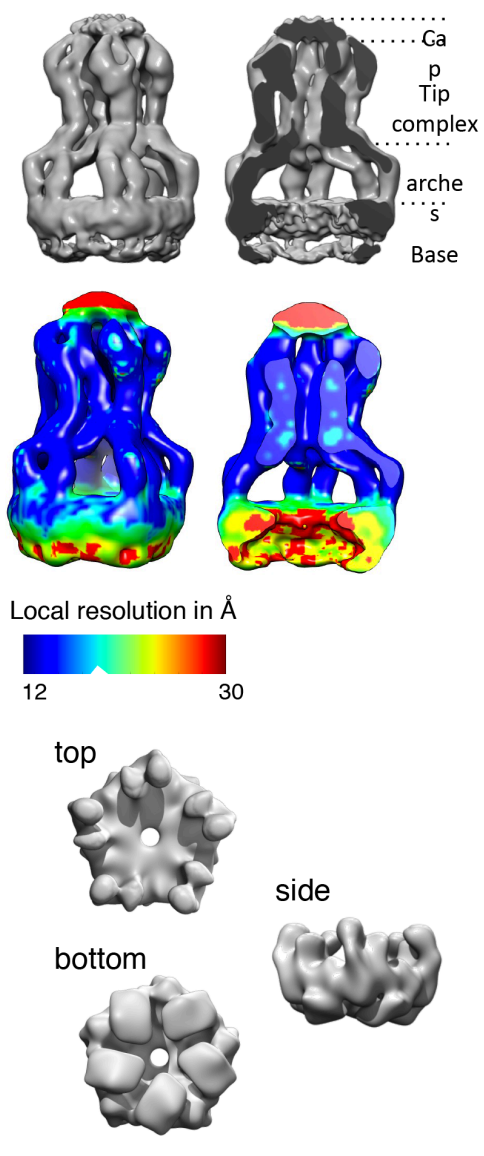

Extended Data Figure 3 


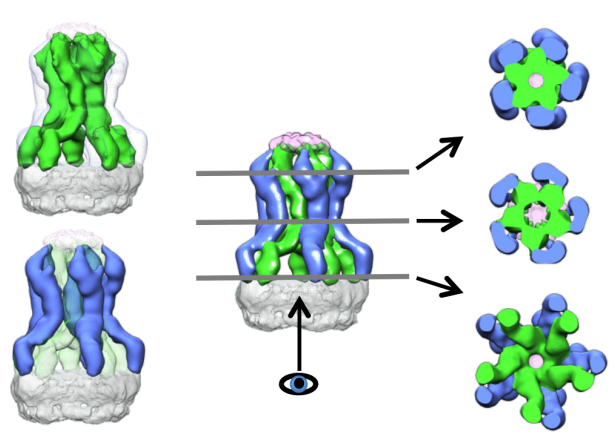

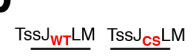
IM OM IM OM

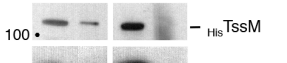

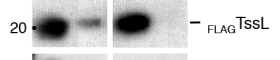
${ }_{15}$ ${ }_{25}--\quad-$ DgIA (IM) ${ }_{37}=-+--$ OMpF $\mathrm{OOM}$

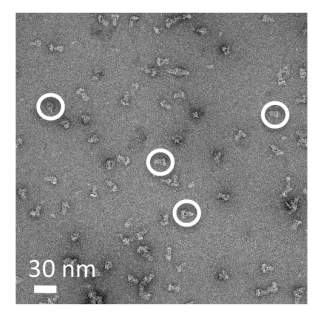

\section{(8) 8 (8) 48}

C
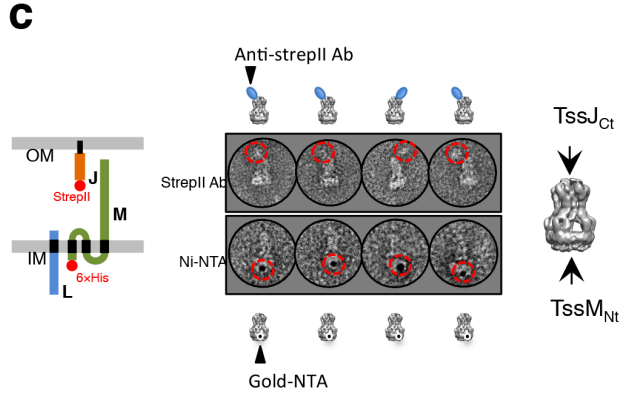

d

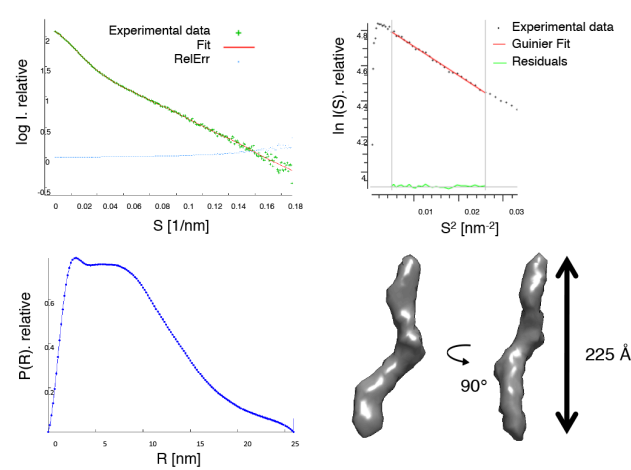

e

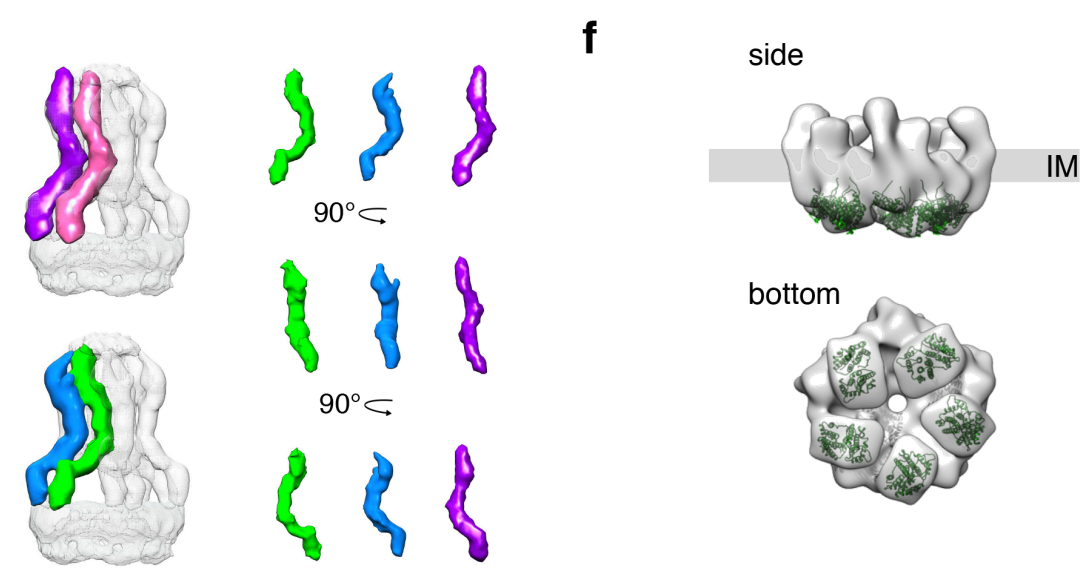

Extended Data Figure 4 


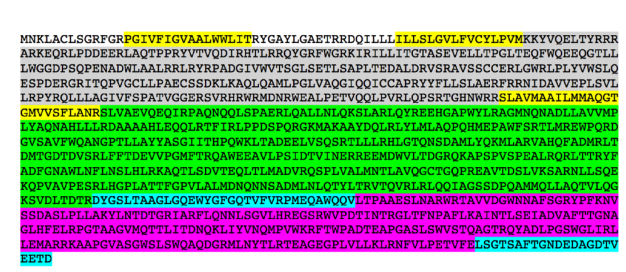

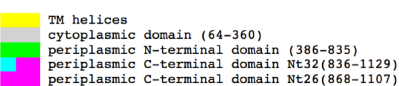

C
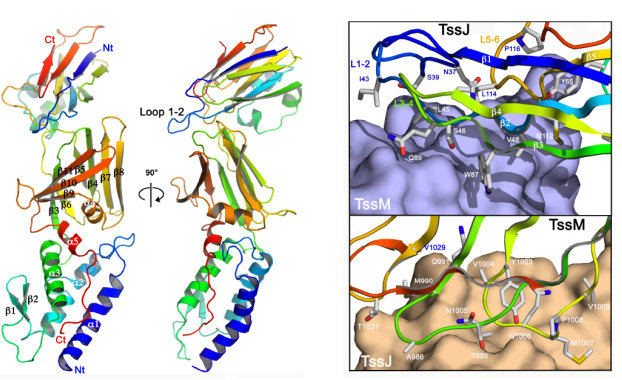

d
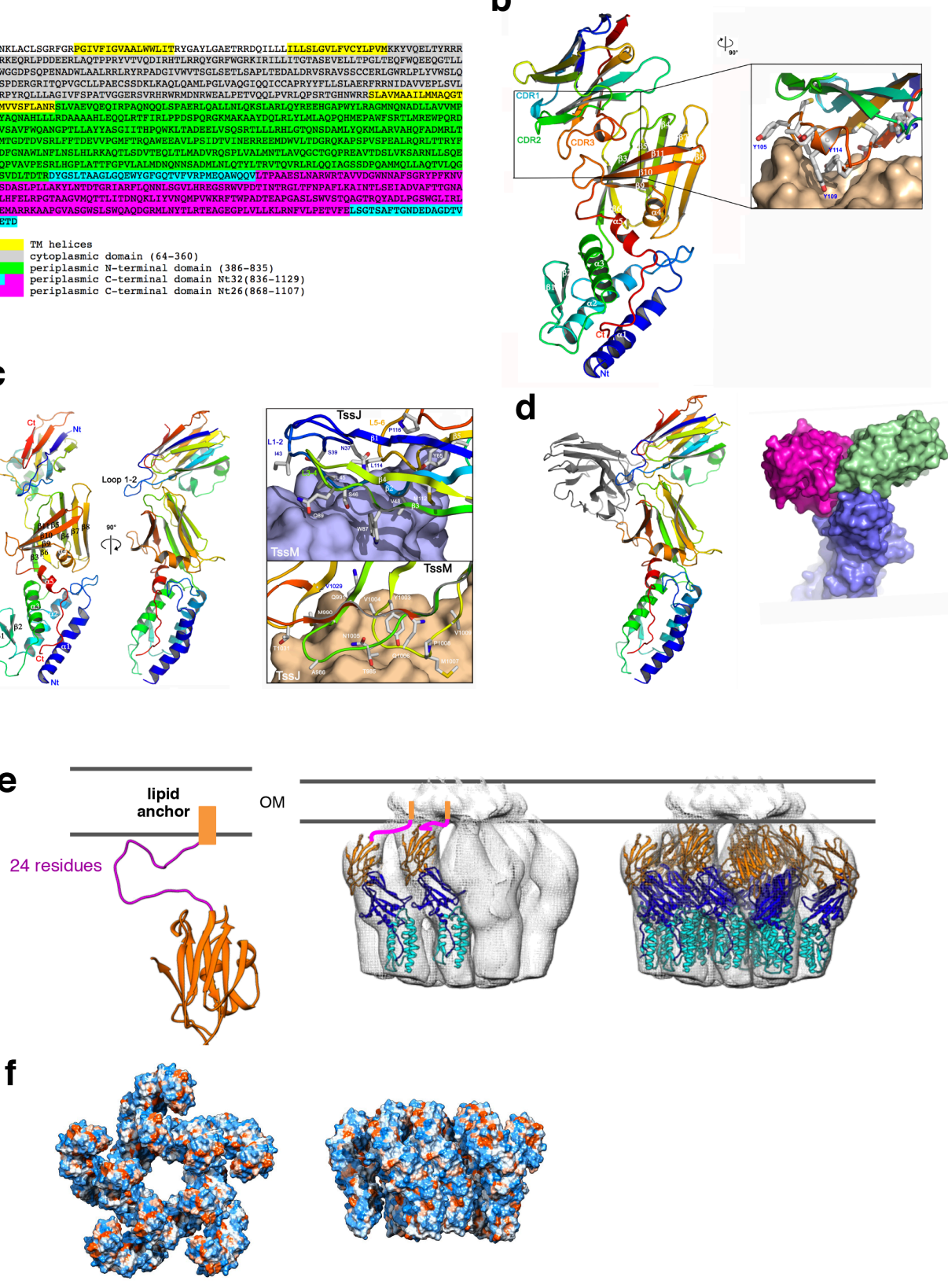

top view

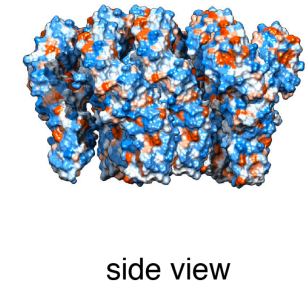

side view

Extended Data Figure 5 


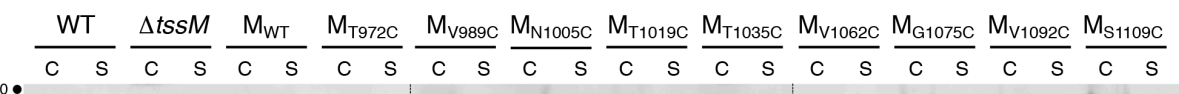

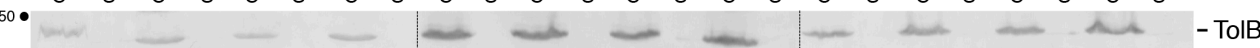

${ }_{30}^{36}:$

16 •

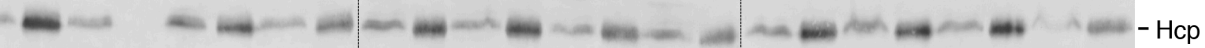

b
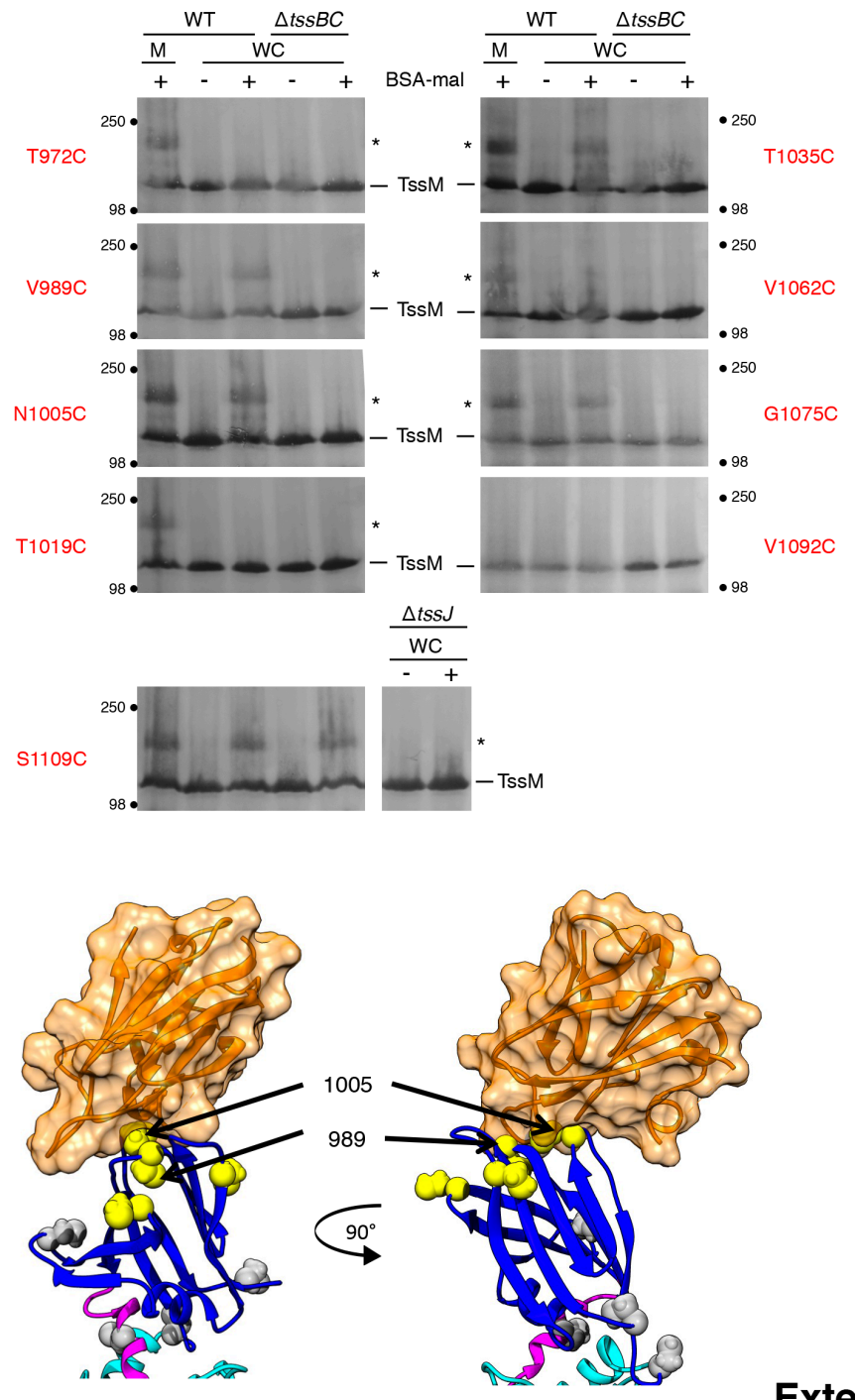

Extended Data Figure 6 
a

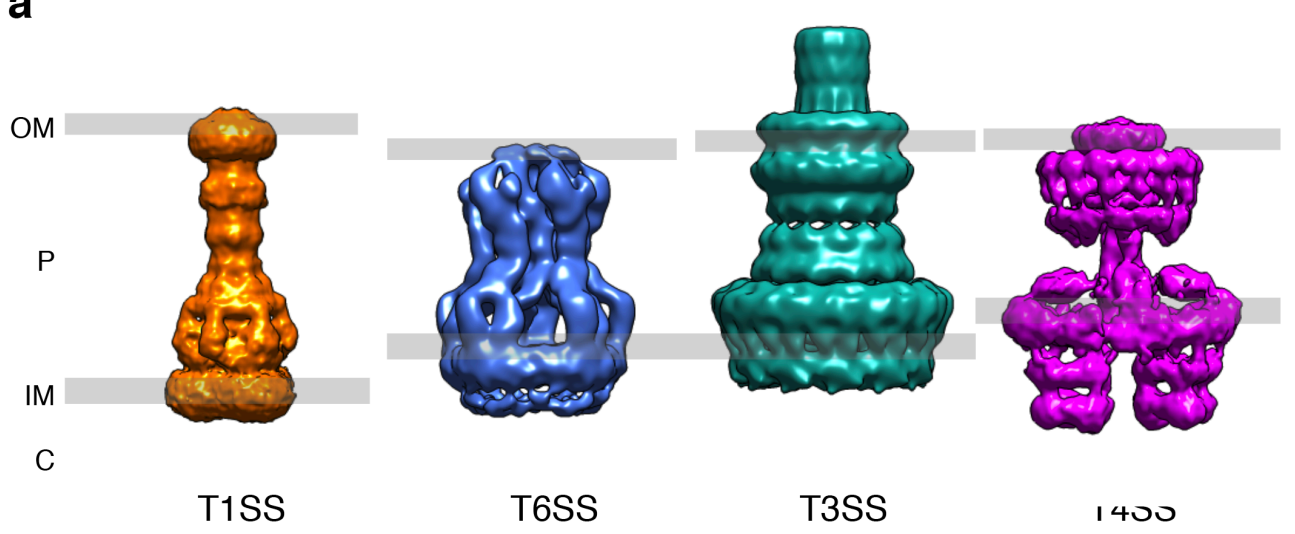

$10 \mathrm{~nm}$

b

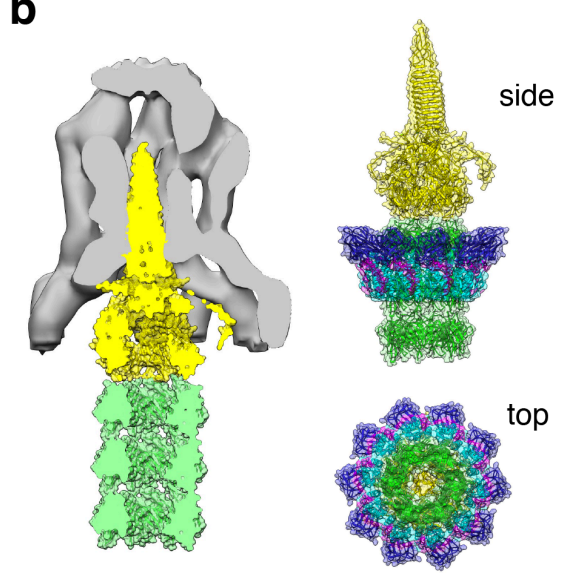

c

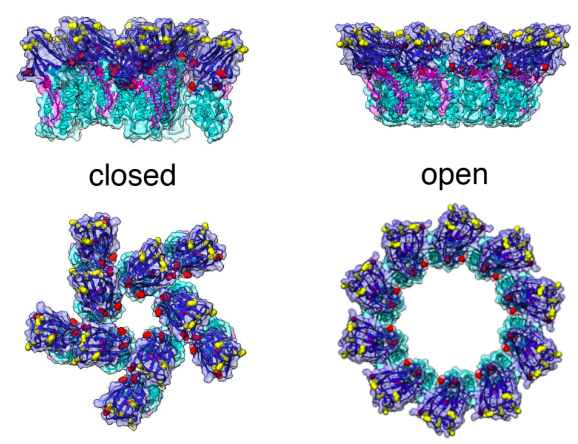

Extended Data Figure 7 